\title{
Uso de métodos Bayesianos nas inferências para a distribuição de Birnbaum-Saunders
}

\author{
Mariano Martínez Espinosa
}

Orientador: Dr. Jorge Alberto Achcar

Dissertação apresentada ao Instituto de Ciências Matemáticas de São Carlos - USP, como parte dos requisitos para a obtenção do título de mestre em "Ciências de Computação e Matemática Computacional".

\author{
¿ão Carlos
}

1992 


\section{AGRADECIMENTOS}

A Deus, pela vida, pelo amor, pela fé e sabedoria.

Ao prof. Dr. Jorge Alberto Achcar, pela excelente orientação deste trabalho.

Ao prof. Dr. Josemar Rodrigues (ICMSC - USP), a profa. Dra. Maria Creusa Bretas Salles (ICMSC - USP) e ao prof. Dr. Carlito Calil Junior ((LaMen) da Escola de Engenharia de São Carlos - USP), pelos comentários e sugestões para a versão final desta dissertação.

A meu filho Bryan, tantas vezes sem a minha companhia, pela absorção no trabalho, e a minha esposa Virlei pela versão portuguesa deste trabalho, pelo incentivo e apoio nos momentos de desânimo, nunca queixando-se pelas horas gastas no trabalho, a você Virlei fonte de inspiração e amparo indispensável para minha vida.

A meus pais Mariano e Eugenia e a minha familia pelo estímulo e carinho, que mesmo de longe sempre me dedicaram, os quais me permitiram vencer mais essa etapa.

A meus sogros Romeu e Enedy e sua familia pela cooperação e estima exemplar.

Ao MRE/ICMSC-USP/CAPES/CNPq, pela oportunidade de dar continuidade à minha formação acadêmica e, pelo apoio financeiro, através do Programa de Estudante-Convênio Pós-Graduação (PEC-PG), Convênio Cultural Brasil-Panamá.

A todas as pessoas que direta ou indiretamente coloboraram na realização deste trabalho. 


\section{RESUMO}

Um dos grandes problemas na área industrial é a ruptura ou falha dos materiais. As falhas são ocasionadas por diversas causas. Uma destas causas é dada por fadiga. A fadiga é a falha estrutural que resulta quando o material é submetido a diferentes esforços dinâmicos repetidos ou flutuantes (STRESS). Um modelo sobre fadiga de materiais muito utilizado na área industrial é dado pela distribuição de Birnbaum-Saunders.

Nesta dissertação, realizamos uma análise Bayesiana para o modelo da distribuição de Birnbaum-Saunders, e para o modelo Log-Linear da distribuição de Birnbaum-Saunders, considerando priori não-informativas diferentes. No modelo Log-Linear da distribuição de Birnbaum-Saunders também consideramos priori informativas. Em ambos os casos encontramos fórmulas simples para as densidades a posteriori marginais e densidades preditivas de interesse, utilizando o método de LAPLACE para integrais, quando não podemos encontrar soluções explícitas.

A metodologia Bayesiana permite aplicações práticas na caracterização de materiais. Tais caracterizações são importantes para prever o desempenho do material sob condições diferentes. Para mostrar a aplicação da metodología Bayesiana consideramos três exemplos numéricos. 


\section{ABSTRACT}

A great industrial problem is related to failures of materials. The failures are related to different causes. One of these causes is given by fatigue. Fatigue is the structural failure resulting from different cyclic loading (Stress). A fatigue model used in the industrial applications is given by the Birnbaum-Saunders distribution.

In this work, we present a Bayesian analysis for the Birnbaum-Saunders distribution, and for a Log-Linear model with the Birnbaum-Saunders distribution considering different non informative priors. In the Log-Linear model of the Birnbaum-Saunders distribution, we also consider informative prior densities. In both cases we find simple expressions for the marginal posterior densities and predictive densities of interest, by using Laplace's method for approximation of integrals, when we can not find explicitly solutions.

The Bayesian approach allows us practical applications in the characterization of materials. These characterizations are important to predict the performance of material under different use conditions. To illustrate the proposed Bayesian methodology, we consider some numerical examples. 


\section{Conteúdo}

1 Introdução 1

1.1 Modelo de Birnbaum-Saunders. . . . . . . . . . . . . 1

1.2 Modelo Log-Linear para a distribuição de Birnbaum-Saunders. . . . . . . 7

1.3 Revisão Bibliográfica (básica). . . . . . . . . . . . . . 14

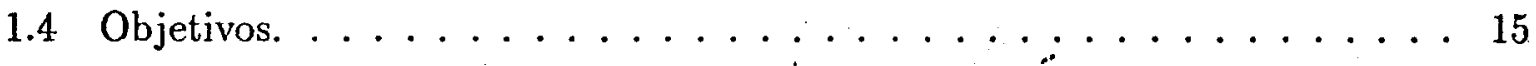

2 Análise Clássica do Modelo de Birnbaum-Saunders 17

2.1 Estimadores de máxima verossimilhança para os parâmetros $\alpha$ e $\beta$. . . . 17

2.2 Inferências sobre os parâmetros $\alpha$ e $\beta . \ldots \ldots \ldots \ldots$

3 Análise Bayesiana do Modelo de Birnbaum-Saunders 24

3.1 Análise Bayesiana assumindo $\alpha$ e $\beta$ desconhecidos. . . . . . . . . . . . 24

3.1.1 Densidades a priori não-informativas para $\alpha$ e $\beta \ldots \ldots . . . . .24$

3.1.2 Densidade a posteriori conjunta para $\alpha$ e $\ldots \ldots \ldots 25$

3.1.3 Densidades a posteriori marginais para $\alpha$ e $\beta \ldots \ldots 26$

3.2 Análise Bayesiana assumindo $\beta$ conheçido. . . . . . . . . . . 28

3.3 Densidade preditiva para uma observação futura . . . . . . . . . . 29 
4 Análise clássica do modelo Log-Linear da distribuição de BirnbaumSaunders

4.1 Estimadores de máxima verossimilhança para os parâmetros $\alpha \mathrm{e} \theta \ldots \ldots 31$

4.2 Inferências sobre os parâmetros $\mathrm{a}$, b e $\alpha \ldots \ldots \ldots \ldots \ldots \ldots$

5 Análise Bayesiana do Modelo Log-Linear para a distribuição de Birnbaum-Saunders (considerando priori não-informativas). $\quad 38$

5.1 Análise Bayesiana assumindo a, b e $\alpha$ desconhecidos. . . . . . . . . 38

5.1.1 Densidade a posteriori conjunta para a, b e $\alpha \ldots \ldots \ldots \ldots 39$

5.1.2 Densidade a posteriori marginal para $\alpha . \ldots \ldots \ldots \ldots 40$

5.1.3 Densidade a posteriori marginal conjunta para a e b. . . . . . . 41

5.2 Uma análise Bayesiana assumindo $\alpha$ conhecido. . . . . . . . . . . . 42

5.2.1 Densidade a posteriori marginal para a e b. . . . . . . . 43

5.2.2 Densidade a posteriori para o tempo medio de vida num nivel de

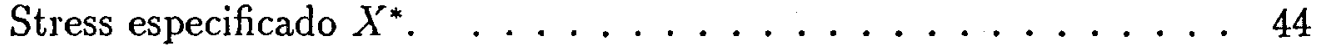

5.2.3 Densidade preditiva para üma observação futura . . . . . . 46

6 Análise Bayesiana do Modelo Log-Linear para a distribuição de Birnbaum-Saunders (considerando densidades a priori informativas). $\quad 48$

6.1 Análise Bayesiana assumindo $\mathrm{a}$, $\mathrm{b}$ e $\alpha$ desconhecidos. . . . . . . . . . 48

6.1.1 Densidade a posteriori conjunta para $a, b$ e $\alpha . \ldots \ldots \ldots \ldots$

6.1.2 Densidades a posteriori marginais para $\alpha, a$ e $b \ldots \ldots \ldots$

6.2 Uma análise Bayesiana assumindo $\alpha$ conhecido. . . . . . . . . . 52

6.2.1 Densidade a posteriori marginal para $a$ e $b \ldots \ldots \ldots \ldots \ldots$

6.2.2 Densidade preditiva para uma observação futura . . . . . . . . 54 
7 Análise da Variancia (ANOVA). $\quad 57$

7.1 Caso geral do modelo. . . . . . . . . . . . . . 57

7.1.1 Estimadores de máxima verossimilhança. . . . . . . . . . . 57

7.1 .2 Estimadores de mínimos quadrados. . . . . . . . . . 62

7.1.3 Estimadores Bayesianos. . . . . . . . . . . . . . 63

7.2 Um caso especial do modelo. . . . . . . . . . . . . . 64

7.2.1 Estimadores de mínimos quadrados. . . . . . . . . . . . . 64

7.2.2 Estimadores de máxima verossimilhança. . . . . . . . . . 66

7.2.3 Inferências sobre os parâmetros $\alpha, \eta, \tau_{1}$ e $u_{1} \ldots \ldots \ldots \ldots 7$

7.2.4 Estimadores Bayesianos. . . . . . . . . . . . . . 69

8 Três exemplos de aplicação $\quad 72$

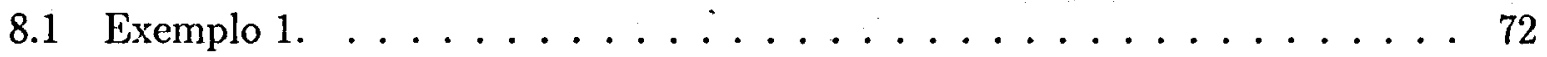

8.1.1 Descrição dos dados. . . . . . . . . . . . . . 72

8.1.2 Verificação gráfica da adequabilidade do modelo. . . . . . . . . 73

8.1.3 Análise clássica do problema. . . . . . . . . . 75

8.1.4 Análise Bayesiana do exemplo 1, assumindo $\alpha$ e $\beta$ desconhecidos. $\quad 76$

8.1.5 Comparação entre as estimativas pontuais e as estimativas por in-

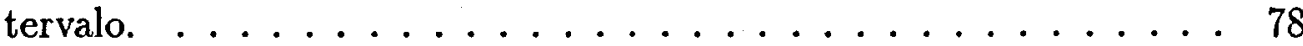

8.1.6 Análise Bayesiana assumindo $\beta$ conhecido $(\beta=132) . \ldots . . . .79$

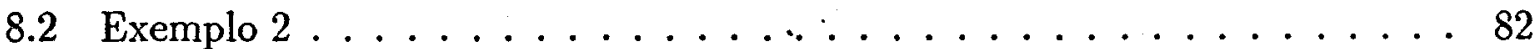

8.2 .1 Descrição dos dados. . . . . . . . . . . . $\$$ \&2

8.2.2 Verificação gráfica da adequabilidade do modelo. . . . . . . . . . $\$ 2$

8.2.3 Análise clássica do problema. ............... 83 
8.2.4 Análise Bayesiana do problema, assumindo a, b e $\alpha$ desconhecidos. 84

8.2.5 Comparação entre as estimativas pontuais e as estimativas por intervalo. ......................... 86

8.2.6 Análise Bayesiana assumindo $\alpha$ conhecido $(\alpha=0.40) \ldots \ldots$. . . 87

8.2.7 Análise Bayesiana considerando densidades a priori informativas e assumindo $\alpha$ conhecido $(\alpha=0.40)$. . . . . . . . . 9 91

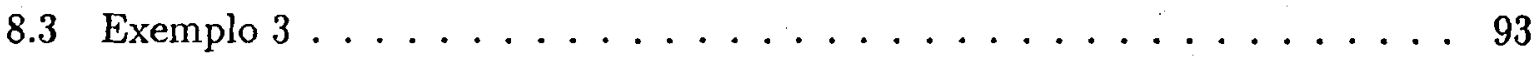

8.3.1 Descrição dos dados. . . . . . . . . . . . . . . 93

8.3.2 Verificação gráfica da adequabilidade do modelo. . . . . . . . . 93

8.3.3 Análise clássica do problema. . . . . . . . . . . . 94

8.3.4 Análise bayesiana do problema. . . . . . . . . . . 95

8.4 Conclusões . . . . . . . . . . . . . . . . . . . 96

-8.5 Direções para futuros trabalhos sobre a distribuição de Birnbaum-Saunders. 97

Apêndice A Método de aproximação de laplace para integrais. 


\section{Capitulo 1}

\section{Introdução}

\subsection{Modelo de Birnbaum-Saunders.}

Um dos grandes problemas na área industrial é a ruptura ou falha dos materiais, as falhas são ocasionadas por diversas causas. Uma dessas causas é a falha por fadiga. A fadiga é a falha estrutural que resulta quando o material é submetido a diferentes esforços dinâmicos repetidos ou flutuantes (STRESS), tais como: forças externas, deformações ou tensões térmicas. Em outras palavras um metal rompe-se por fadiga, quando a tensão cíclica aplicada a ele, tem uma flutação suficientemente grande e é maior que um valor característico de cada metal, denominado limite de fadiga, o qual pode ser determinado mediante um ensaio de fadiga.

A ruptura geralmente ocorre quando o número de ciclos de tensão aplicada é suficientemente grande. No entanto, muitos outros fatores afetam a ruptura por fadiga, tornando muito extenso o seu estudo. Neste trabalho, consideramos apenas algumas ideias "elementares" do estudo de fadiga, sem entrar em detalhes a respeito do estudo completo da fadiga dos metais. Para maiores pormenores ver por exemplo, Souza, 1982.

Um modelo sobre fadiga de materiais muito utilizado na área industrial é dado pela distribuição de Birnbaum-Saunders. Esta distribuição, baseada em dois parâmetros foi proposta por Birnbaum e Saunders em 1969, a qual foi desenvolvida usando alguns resultados da teoria de renovação (ver Birnbaum e Saunders,1968), por meio de uma idealização do número de ciclos necessários parà a ruptura por fadiga. 
Antes de elucidar como surge esta distribuiçẫo é preciso definirmos dois têrmos muito utilizados no estudo de fratura por fadiga: ciclo e tensão. Um ciclo é um intervalo de tempo no qual alguma sequência de tensões é aplicada na amostra, em forma cíclica (cíclico é todo processo que oscila periódicamente entre dois extremos), regularmente numa mesma ordem. Tensão é o quociente de intensidade de uma força pela área de superficie da amostra, isto é, tensão é a força que se aplica num ciclo.

Para ilustrar brevemente como surge esta distribuição, consideremos uma amostra de um material padronizado que é submetida a uma sequência de $\mathrm{m}$ tensões: $l_{1}, \ldots, l_{m}$, onde cada uma das quais pode causar uma fratura por fadiga. A fadiga se inicia em um ponto, e se propaga de modo concêntrico e vagarosamente através do material. $\mathrm{O}$ progresso da fadiga reduz a área resistente efetiva do material, aumentando em consequência, a tensão real na estrutura e acarretando a fratura na seção afetada. Também assumiremos que a tensão é cíclica, tal que se em cada ciclo alguma sequência de tensões (e em alguma ordem) é aplicada na amostra, e será de maneira idêntica ao ciclo anterior.

O esquema de tensões pode ser representado da seguinte forma:

$$
l_{1}, \ldots, l_{m}, l_{m+1}, \ldots, l_{2 m}, \ldots, l_{j m+1}, \ldots, l_{j m+m} .
$$

Ciclo 1, Ciclo 2, $, \ldots, \operatorname{Ciclo}(j+1), \quad$.

onde para todo $i=1,2, \ldots, m, l_{j m+i}=l_{k m+i}$, para todo $j \neq k ; j, k=1, \ldots$, .

Além disso, Birnbaum e Saunders assumem que a tensão é continua e ordenada, e a tensão é vista como uma função unimodal continua (ver Birnbaum e Saunders, 1968).

Então, tensões continuas implicam que para todo $i=1,2, \ldots, m$

$$
l_{i+1}(0)=l_{i}(1) \text {. }
$$

isto é, supondo que o zero (0) é o instante inicial do ciclo, e um (1) o instante final do ciclo. Então o fim do i-ésimo ciclo coincide com o inicio do $(i+1)$-ésimo ciclo.

Fisicamente isto é razoavel para supor que a falha por fadiga é devido ao inicio, crescimento e última expansão de uma abertura. Para cada oscilação esta abertura é estendida para algum total, a qual é uma função aleatória. A aleatorização é devida a variaçôes no material, a magnitude da tensão aplicada e um certo número de tensões anteriores, e talvez ao aumento atual das expansões causadas por tensões anteriores nesse ciclo.

Tendo estabelecido o procedimento da estrutura física, é necessário fazer duas suposições de natureza estatística. 
Suposição 1.1 incremento da expansão da abertura $X_{i}$ devido as tensões $l_{i}$ no $j$-ésimo ciclo é uma variável aleatória com uma distribuição que depende do tamanho da abertura no inicio do ciclo, do aumento atual da expansão causada pelas oscilaçóes e de um certo número de tensões que tem precedido em esse ciclo.

Esta suposição é razoável para o estudo de fadigas em aeronaútica (ver por exemplo, Birnbaun e Saunders, 1969-a), e igualmente em outras aplicações.

O crescimento total da abertura durante o $(j+1)$-ésimo ciclo da tensão é:

$$
Y_{j+1}=X_{j m+1}+X_{j m+2}+\ldots+X_{j m+m},
$$

para $j=0,1, \ldots$, onde $X_{j m+i}$ é o crescimento da abertura (possivelmente microscópica) para a tensão $l_{i}$ aplicada na i-ésima oscilação do $(j+1)$-ésimo ciclo.

Esta suposição garante que, sem consideração da dependência dentro de sucessivos crescimentos aleatórios devido a tensões em um particular ciclo, o total aleatório do crescimento da abertura é independente de um ciclo para outro.

Suposição 1.2 O crescimento total da abertura $Y_{j}$ devido ao j-ésimo ciclo é uma variável aleatória com média $\mu$ e variancia $\sigma^{2}$, para $j=1,2, \ldots$, .

Sob uma aplicação repetida de n ciclos de tensões, o crescimento total de uma abertura numa amostra pode ser escrito da seguinte forma:

$$
W_{n}=\sum_{j=1}^{n} Y_{j},
$$

a qual tem função de distribuição dada por,

$$
F_{n}(w)=P_{r}\left(W_{n} \leq \omega\right),
$$

para $n=1,2, \ldots$ Isto simplesmente utilizando principios básicos de probabilidade (ver por exemplo, Ross, 1976).

Seja $\mathrm{k}$ uma variável aleatória de um valor inteiro positivo, denotando o menor número de ciclcos para os quais $W_{n}$ excede um valor crítico $\omega$, isto é, o número de ciclos até a falha da amostra. Então, 


$$
P_{r}(K \leq n)=P_{r}\left(W_{n}>\omega\right)
$$

isto implica que,

$$
P_{r}(K \leq n)=1-P_{r}\left\{\sum_{j=1}^{n} Y_{j} \leq \omega\right\}
$$

Pela suposição (1.2) $Y_{j}$ tem média $\mu$ e variancia $\sigma^{2}$; assim os $Y_{j}$-ésimos podem ser normalizados, e temos:

$$
P_{r}(K \leq n)=1-\operatorname{Pr}\left\{\frac{\sum_{j=1}^{n} Y_{j}-n \mu}{\sigma \sqrt{n}} \leq \frac{\omega-n \mu}{\sigma \sqrt{n}}\right\} .
$$

A suposição (1.1) garante a independencia das variáveis aleatórias $Y_{j}$, e se n é grande (um critério que pode ser facilmente satisfeito no estudo de fadigas) o Teorema do Limite Central pode ser aplicado (ver por exemplo, Kalfleisch, 1985).

Pelo Teorema do Limite Central e pela simetría da distribuição normal, temos:

$$
P(K \leq n)=\Phi\left\{\frac{n \mu}{\sigma \sqrt{n}}-\frac{\omega}{\sigma \sqrt{n}}\right\},
$$

onde $\Phi(x)$ é a função de distribuição acumulada de uma distribuição normal padrão, isto é,

$$
\Phi(x)=\frac{1}{\sqrt{2 \pi}} \int_{-\infty}^{x} e^{-s^{2} / 2} d s .
$$

Podemos naturalmente estender a variável aleatória $\mathrm{K}$ para uma variável aleatória positiva continua $\mathrm{T}$, onde $\mathrm{T}$ denota o tempo de vida da fadiga (ver por exemplo, Mann, Schafer e Singpurwalla, 1974) com uma função de distribuição $F_{T}(t)$. Portanto, substituindo $\mathrm{n}$ (n é o número de ciclos) por $\mathrm{t}$, a função de distribuição acumulada de $\mathrm{T}$ é dada por:

$$
F_{T}(t)=P_{r}(T \leq t)=\Phi\left\{\frac{\mu \sqrt{t}}{\sigma}-\frac{\omega}{\sigma \sqrt{t}}\right\} .
$$

Assim definindo

e

$$
\alpha=\frac{\sigma}{\sqrt{\mu \omega}}
$$

$$
\beta=\frac{\omega}{\mu}
$$


temos,

$$
F_{T}(t)=\Phi\left[\frac{1}{\alpha}\left\{\left(\frac{t}{\beta}\right)^{1 / 2}-\left(\frac{\beta}{t}\right)^{1 / 2}\right\}\right]
$$

para $t>0, \alpha>0$ e $\beta>0$.

Observar que (1.11) é a função distribuição do tempo de vida da fadiga com parâmetros $\alpha$ e $\beta$ (distribuição de Birnbaum-Saunders). Os parâmetros $\alpha$ e $\beta$ são respectivamente os parâmetros de forma e escala.

De (1.11) segue que,

$$
Z=\frac{1}{\alpha}\left\{\left(\frac{T}{\beta}\right)^{1 / 2}-\left(\frac{\beta}{T}\right)^{1 / 2}\right\}
$$

é normalmente distribuida com média zero e variancia 1. Portanto a função de densidade de probabilidade de $\mathrm{T}$ é dada por,

$$
f(t ; \alpha, \beta)=\frac{(t+\beta) \exp \left[\frac{-1}{2 \alpha^{2}}\left\{\frac{t}{\beta}+\frac{\beta}{t}-2\right\}\right]}{2 \sqrt{2 \pi} \alpha \beta^{1 / 2} t^{3 / 2}},
$$

para $t>0 ; \alpha, \beta>0$.

Observar que,

$$
\frac{t+\beta}{\beta^{1 / 2} t^{3 / 2}}=\frac{t^{2}-\beta^{2}}{\beta t^{2}\left\{\left(\frac{t}{\beta}\right)^{1 / 2}-\left(\frac{\beta}{t}\right)^{1 / 2}\right\}},
$$

por conseguinte (1.13) pode ser, também escrita da seguinte forma:

$$
f(t ; \alpha, \beta)=\frac{\left(t^{2}-\beta^{2}\right) \exp \left[\frac{-1}{2 \alpha^{2}}\left\{\frac{t}{\beta}+\frac{\beta}{t}-2\right\}\right]}{2 \sqrt{2 \pi} \alpha \beta t^{2}\left[\left(\frac{t}{\beta}\right)^{1 / 2}-\left(\frac{\beta}{t}\right)^{1 / 2}\right]},
$$

para $t>0 ; \alpha, \beta>0$.

A média de $\mathrm{T}$ é dada por,

$$
E[T]=\beta\left[1+\frac{\alpha^{2}}{2}\right]
$$

e a variancia de $\mathrm{T}$ por,

$$
\operatorname{Var}[T]=(\alpha \beta)^{2}\left[1+\frac{5}{4} \alpha^{2}\right]
$$


(Ver por exemplo, Birnbaum e Saunders, 1969-a).

$\mathrm{Na}$ figura 1.1 temos o gráfico da densidade (1.13), para varios valores de $\alpha$ $(\alpha=0.10,0.25,0.50,1.0,1.5,2.1), \operatorname{com} \beta=1$. Observe corno a distribuição vai tornandose assimétrica a medida que o valor de $\alpha$ aumenta.

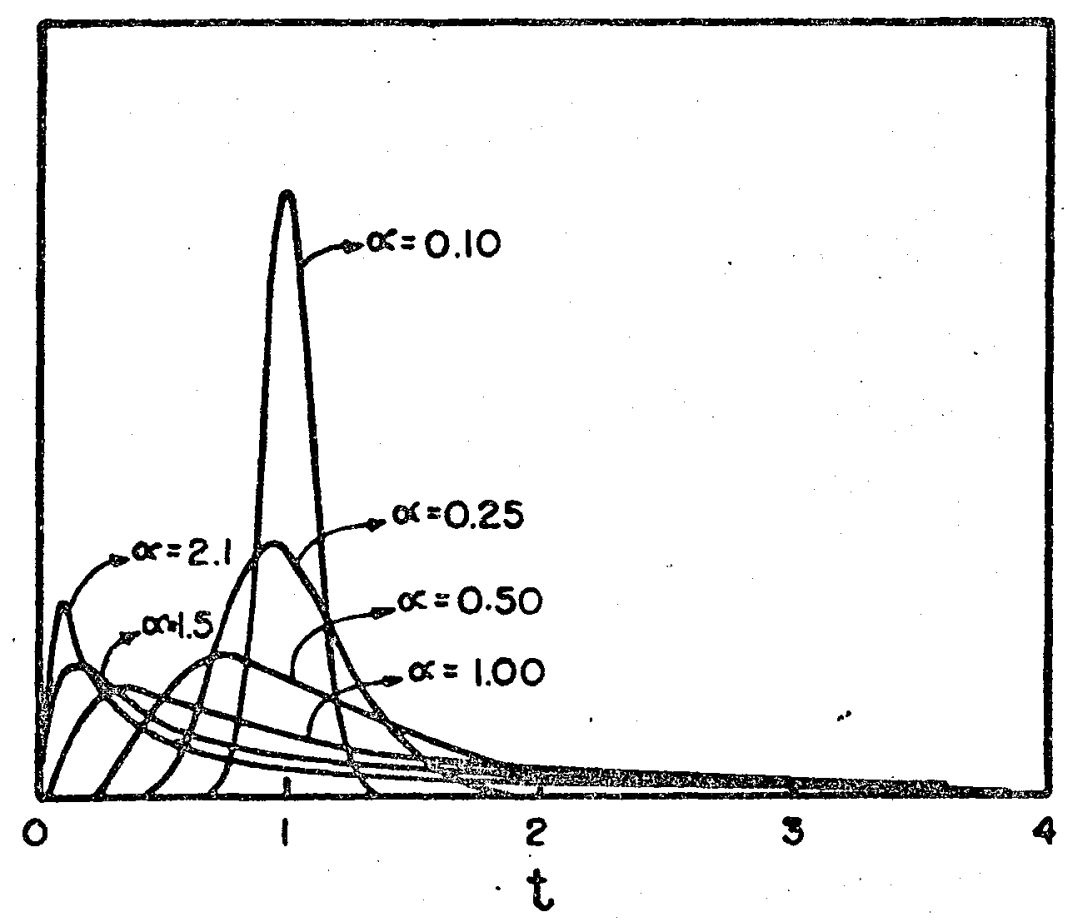

Figura 1.1 Gráfico da densidade (1.13) para varios valores de $\alpha$, com $\beta=1$. 


\subsection{Modelo Log-Linear para a distribuição de Birn- baum-Saunders.}

O estudo de fadiga é de grande importância para projetos de fabricação de peças sujeitas a tensões cíclicas. No entanto o tempo e o custo de ensaios de fadiga de materiais, em geral é muito elevado. Uma alternativa para reduzir o tempo e custo dos ensaios por fadiga é utilizar o modelo Log-Linear para a distribuição de BirnbaumSaunders (ver, Rieck e Nedelman, 1991), o qual pode ser usado para o estudo de fadiga. O experimento de fadiga pode ser realizado na própria peça, isto é, utilizando corpos de prova (amostras), caso se possua uma máquina de fadiga adequada, reproduzindo no ensaio da melhor maneira possivel os esforços a que ela é submetida na prática, nesse caso testando o material em sí, sem verificar os efeitos das características particulares na própria peça. Estes ensaios ou testes são conhecidos na área de Estatística como testes de vida acelerados.

O modelo Log-Linear pode ser motivado por aplicações na caracterização de materiais. Os engenheiros em geral fornecem regras empíricas descrevendo o tempo de falha dos materiais, submetidos a diferentes padrões de tensões cíclicas. Com estas regras empíricas, os materiais podem ser caracterizados pelos valores dos parâmetros. Tais caracterizações são importantes para prever o desempenho do material sob condições diferentes. Por exemplo, frequentemente estamos interessados em prever o tempo da fadiga para pequenos niveis de Stress. Para conduzir um teste com pequenos níveis de Stress em geral gastamos muito tempo e dinheiro. Potanto os engenheiros consideram falhas de amostras com altos niveis de Stress, e daí inferem os tempos de falhas para pequenos níveis de Stress, isto é, utilizando testes de vida acelerados.

Para ilustrar brevemente como surge este modelo, suponha que corpos de prova de um metal são submetidos a repetidas tensões, com níveis de tensões diferentes aos valores admissiveis, estaticamente. Seja $\mathrm{S}$ a tensão por ciclo (Stress) e seja $\mathrm{N}$ o número de ciclos de tensões suportados pelo corpo de prova até a fratura (até a amostra falhar). Esse número é anotado na própria máquina de fadiga e representa a soma do número de ciclos para iniciar uma ruptura de fadiga mais o número de ciclos para propagar a ruptura através do material. Caso o material não se rompa, o número, $N$, também pode indicar o número de ciclos.

A curva tensão-número de ciclos, também chamada curva de Wölher ou simplesmente curva S-N (S símbolo de tensão (Strèss)) é a forma mais usual para apresentar os resultados dos ensaios de fadiga (ver por exemplo, Souza, 1982). Nessa curva, o número $\mathrm{N},($ ou $\log N)$ é colocado no eixo das ordenadas e no eixo das abscisas colocamos a tensão 
máxima $S$, (generịcamente a tensão máxima é a resistência máxima suportada por um material à fadiga, para um dado número de ciclos, sem romper) que também pode ser expressa na escala logarítmica (muitos autores utilizam $W_{c}$ para expressar a tensão máxima). A escala logarítmica facilita a comparação de dados, pois fornece curvas de diversos materiais com a mesma forma, além de facilitar e diminuir a escala de N. Assim encontramos regras empíricas distintas de grande utilidade na área industrial, usualmente na seguinte forma:

$$
\ln (N)=a+b X
$$

onde X é o logaritmo da tensão, isto é $\ln (S)$ ou $\ln \left(W_{c}\right)$, A escolha de $\mathrm{X}$ depende do material e das condições testadas.

$\mathrm{Na}$ prática estimar os parâmetros a e b de (1.17) é um problema estatístico. Para fornecer uma estrutura estatística adequada para estimação, são feitas as seguintes suposições (ver, Rieck e Nedelman, 1991):

Suposição 1.3 O número de ciclos para a falha tem uma distribuição de BirnbaumSaunders; onde $N$ representa a mediana desta distribuição.

Suposição 1.4 O parâmetro de forma $\alpha$ é independente da tensão por ciclo.

Assim se $T$ tem uma distribuição de Birnbaum-Saunders com mediana $\beta$ e com parâmetro de forma $\alpha$, e se $K>0$ é uma constante, então KT tem uma distribuição de BirnbaumSaunders com mediana $K \beta$. Isto é, se as suposições (1.3) e (1.4) são válidas, então (1.17) pode ser representada da seguinte maneira:

$$
N=\exp \{a+b X\} \delta
$$

onde $\delta$ tem uma distribuição de Birnbaum-Saunders. Aplicando logaritmo em (1.18) temos:

$$
\ln (N)=a+b X+\ln \delta
$$

Assim (1.19) é o modelo Log-Linear na forma aditiva, onde $\ln \delta$ tem uma distribuição Senh-Normal (distribuição seno-hiperbólico-Normal).

Antes de estabelecermos o relacionamento entre a distribuição de BirnbaumSaunders e a distribuição Senh-Normal, daremòs algumas informações gerais sobre a distribuição Senh-Normal, necessárias para um melhor entendimento deste relacionamento. 
Em sua forma geral se Y é uma variável aleatória com função densidade de probabilidade dada por,

$$
f(y)=\left\{\frac{2}{2 \sqrt{2 \pi} \alpha}\right\} \cosh \left\{\frac{y-\gamma}{v}\right\} \exp \left[\frac{-2}{\alpha^{2}} \operatorname{senh}^{2}\left\{\frac{y-\gamma}{v}\right\}\right],
$$

para $-\infty<y<\infty ; \alpha, v>0$ e $-\infty<\gamma<\infty$, então Y tem uma distribuição SenhNormal, a qual pode ser denotada por $\mathrm{SN}(\alpha, \gamma, v)$, onde $\gamma$ é um parâmetro de locação, $v$ é um parâmetro de escala e $\alpha$ é um parâmetro de forma. A seguir mencionaremos algumas das principais propriedades desta distribuição:

1- A distribuição é simétrica em torno do parâmetro de locação $\gamma$.

2- A distribuição é fortemente unimodal para $\alpha \leq 2$ e bimodal para $\alpha>2$.

1991).

3- A média da distribuição é dada por $E[y]=\gamma$. (ver, Rieck e Nedelman,

Rieck e Nedelman em 1991 afirmam que valores de $\alpha>2$ não são usuais na prática. Observe que se $\alpha>1$, temos um coeficiente de variação maior do que 1 , o qual não é tolerado em aplicações industriais.

A seguir estabeleceremos o relacionamento entre a distribuição Senh-Normal e a distribuição de Birnbaum-Saunders.

Seja T uma variável aleatória, com distribuição de Birnbaum-Saunders, com parâmetros $\alpha$ e $\beta$, e considere a transformação $Y=\ln (T)$. Então,

$$
T=e^{Y} \quad e \quad \frac{\partial Y}{\partial T}=\frac{1}{T},
$$

o que implica que,

$$
\left|\frac{\partial T}{\partial Y}\right|=e^{Y},
$$

onde $\frac{\partial T}{\partial Y}$ é o Jacobiano da transformação.

Portanto, utilizando (1.21), (1.22) e (1.20) a função de densidade de probabilidade para a variável aleatória $\mathrm{Y}$ é dada por:

$$
f(y)=\frac{e^{y}\left(e^{2 y}-\beta^{2}\right) \exp \left[\frac{-1}{2 \alpha^{2}}\left\{\frac{e^{y}}{\beta}+\frac{\beta}{e^{y}}-2\right\}\right]}{2 \sqrt{2 \pi} \alpha \beta e^{2 y}\left[\left(\frac{e^{y}}{\beta}\right)^{1 / 2}-\left(\frac{\beta}{e^{y}}\right)^{1 / 2}\right]}
$$




$$
\begin{aligned}
& \text { onde }-\infty<y<\infty, \alpha>0 \text { e } \beta>0 \\
& \text { Definindo } \gamma=\ln \beta \text {, temos: } \\
& \quad \exp \left[\frac{-1}{2 \alpha^{2}}\left\{\frac{e^{y}}{\beta}+\frac{\beta}{e^{y}}-2\right\}\right]=\exp \left[\frac{-2}{2 \alpha^{2}}\left\{\frac{\left.e^{(y-\gamma}\right)+e^{-(y-\gamma)}-1}{2}\right\}\right] .
\end{aligned}
$$

A seguir apresentamos alguns resultados das funções trigonométricas hiperbólicas (ver por exemplo, Leithold, 1972):

\section{Resultado 1.1}

$$
\cosh x=\frac{e^{x}+e^{-x}}{2}
$$

\section{Resultado 1.2}

$$
\operatorname{senh} x=\frac{e^{x}-e^{-x}}{2}
$$

Resultado 1.3

$$
\operatorname{senh}^{2} x=\frac{1}{2} \cosh 2 x-\frac{1}{2}
$$

Resultado $\mathbf{1 . 4}$

$$
\operatorname{senh} 2 x=2 \operatorname{senh} x \cosh x .
$$

\section{Resultado $\mathbf{1 . 5}$}

$$
\cosh ^{2} x-\operatorname{senh}^{2} x=1
$$


Resultado 1.6

$$
\cosh 2 x=2 \cosh ^{2} x-1
$$

Portanto, utilizando os resultados (1.1) e (1.3), temos,

$$
\exp \left[\frac{-1}{2 \alpha^{2}}\left\{\frac{e^{y}}{\beta}+\frac{\beta}{e^{y}}-2\right\}\right]=\exp \left\{\frac{-2}{\alpha^{2}} \operatorname{senh}^{2}\left(\frac{y-\gamma}{2}\right)\right\} .
$$

Igualmente usando o resultado (1.2), temos:

$$
\frac{e^{y}\left(e^{2 y}-\beta^{2}\right)}{\beta e^{2 y}\left\{\frac{e^{y / 2}}{\beta^{1 / 2}}-\frac{\beta^{1 / 2}}{e^{y / 2}}\right\}}=\frac{\operatorname{senh}(y-\gamma)}{\operatorname{senh}\left(\frac{(y-\gamma)}{2}\right)} .
$$

Pelo resultado (1.4),

$$
\operatorname{senh}(y-\gamma)=2 \operatorname{senh}\left[\frac{y-\gamma}{2}\right] \cosh \left[\frac{y-\gamma}{2}\right] .
$$

Portanto (1.24) pode ser dado na seguinte forma:

$$
\frac{e^{y}\left(e^{2 y}-\beta^{2}\right)}{\beta e^{2 y}\left\{\frac{e y / 2}{\beta^{1 / 2}}-\frac{\beta^{1 / 2}}{e^{y / 2}}\right\}}=2 \cosh \left[\frac{y-\gamma}{2}\right]
$$

Assim (1.23) é dada por:

$$
f(y)=\left\{\frac{2}{2 \sqrt{2 \pi} \alpha}\right\} \cosh \left\{\frac{y-\gamma}{2}\right\} \exp \left[\frac{-2}{\alpha^{2}} \operatorname{senh}^{2}\left\{\frac{y-\gamma}{2}\right\}\right],
$$

para $-\infty<y<\infty, \alpha>0$, e $-\infty<\gamma<\infty$. Observar que a função densidade de probabilidade da expressão (1.25) é um caso particular da distribuição Senh-Normal (1.20), onde $\alpha$ é um parâmetro de forma, $\gamma=\ln (\beta)$ é um parâmetro de locação e $v=2$ é um parâmetro de escala. 
Assim demonstramos que se $\mathrm{T}$ tem uma distribuição de Birnbaum-Saunders, com parẩmetros de forma $\alpha$ e parâmetro de escala $\beta$, então a variável aleatória $Y=\ln (T)$ tem uma distribuição Senh-Normal com função densidade de probabilidade dada por (1.25).

Na figura 1.2 temos o gráfico da densidade (1.25), para alguns casos especiais de $\alpha(\alpha=0.10,0.25,0.50,1.0)$, com $\beta=1$, isto é $\gamma=0$. Observe como a variabilidade aumenta quando $\alpha=1.0$.

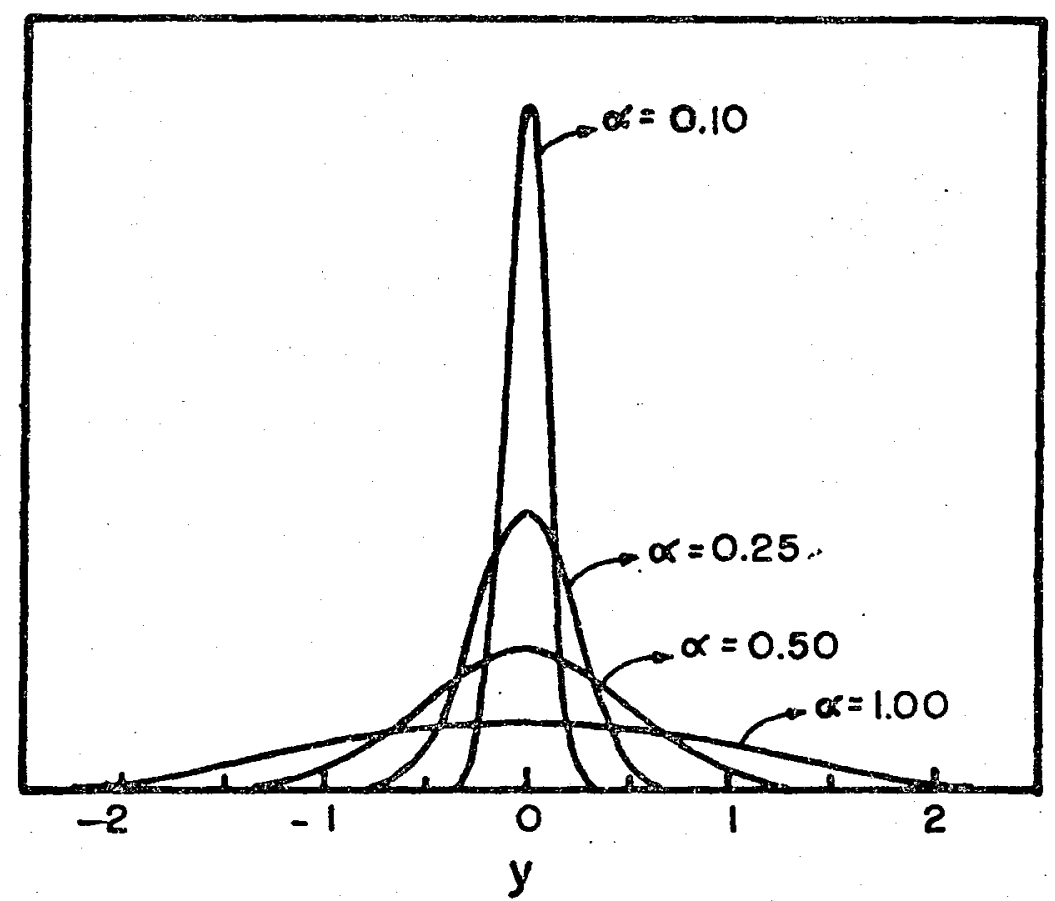

Figura 1.2 Gráfico da densidade (1.25) para algums valores de $\alpha<2.0$, com $\gamma=0$. 
Similarmente na figura 1.3 apresentamos o gráfico da densidade (1.25) para $\alpha=2.1, \operatorname{com} \gamma=0$.

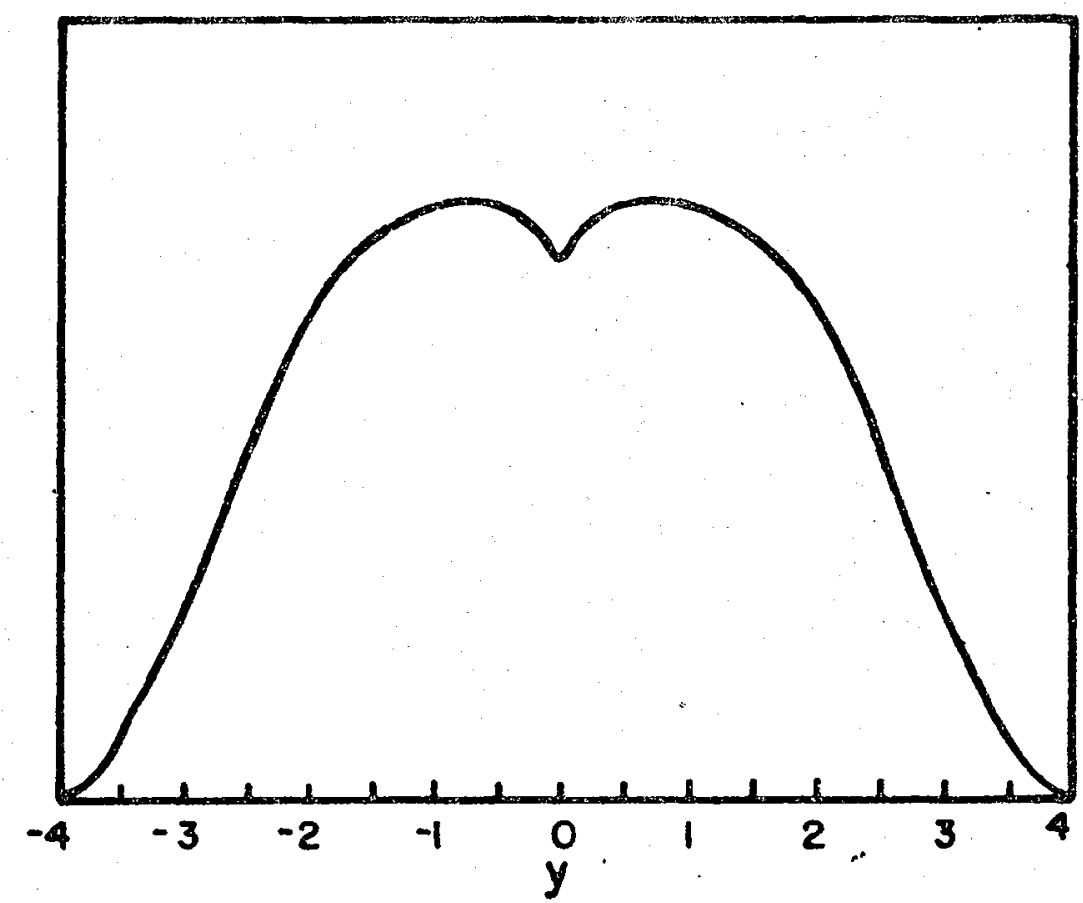

Figura 1.3 Gráfico da densidade (1.25) para $\alpha=2.1$, com $\gamma=0$.

Neste gráfico podemos notar que a distribuição Seno-hiperbólico-Normal tornase bimodal para valores de $\alpha>2$, (como foi comentado, nas suas propriedades principais) o qual pode causar multiplos estimadores de máxima verossimilhança. 


\subsection{Revisão Bibliográfica (básica).}

O estudo da fadiga de materiais tem sido uma área pesquisada por muitos autores. Uma extensa bibliografía sobre o estudo de fadiga de materiais pode ser encontrada em Souza (1982).

Birnbaum e Saunders (1968) apresentam suposições da teoria de renovação, por meio de uma idealização do número de ciclos necessários para a ruptura por fadiga. Birnbaum e Saunders (1969-a) apresentam algumas propriedades básicas da distribuição de Birnbaum-Saunders, e em (1969-b) acham os estimadores de máxima verossimilhança conjuntos para os parâmetros. Engelhardt, Bain e Wright (1981) fornecem testes de hipóteses e intervalos de confiança para os parâmetros da distribuição de Birnbaum-Saunders baseados nos estimadores de máxima verossimilhança. Fries e Bhattacharyya (1983) sugerem que a distribuição de Birnbaum-Saunders, pode ser de grande utilidade para o estudo de planejamento de experimentos na área de confiabilidade e testes de sobrevivência industriais. Achcar (1991) realiza uma análise Bayesiana da distribuição de Birnbaum-Saunders considerando densidades a priori não informativas para os parâmetros e usa o método de LAPLACE (ver por exemplo, Kass, Tierney e Kadane, 1990) para aproximar as integrais, quando não consegue obter as soluçóes explícitas das densidades a posteriori de interesse.

Rieck e Nedelman (1991), formulam e apresentam um modelo Log-Linear para a distribuição de Birbaum-Saunders, o qual pode ser utilizado para o estudo de fadiga em testes de vida acelerados. Achcar e Martínez (1992) desenvolvem uma análise Bayesiana do modelo Log-Linear para a distribuição de Birnbaum-Saunders, considerando uma variável Stress, assumindo densidades a priori não informativas para os parâmetros e utilizando o método de Laplace (ver por exemplo, Kass, Tiemey e Kadane, 1990) para aproximar as integrais quando não é possivel obter as soluções explícitas para as densidades a posteriori de interesse. 


\subsection{Objetivos.}

O objetivo principal deste trabalho é realizar uma análise Bayesiana para o modelo da distribuição de Birnbaum-Saunders, e para o modelo Log-Linear da distribuição de Birnbaum-Saunders considerando uma variável Stress. Para representar o grau de conhecimento sobre os parâmetros no modelo da distribuição de Birnbaum-Saunders utilizaremos densidades a priori não-informativas. No modelo Log-Linear da distribuição de Birnbaum-Saunders usaremos densidades a priori não-informativas e informativas, devido a sua grande utilização em projeto de fabricação de peças sujeitas a falhas por fadiga. Também usaremos o método de LAPLACE (ver por exemplo, Kass, Tierney e Kadane, 1990) para aproximar as integrais quando não conseguimos soluções analíticas explicitas das densidades a posteriori de interesse (ver capítulos 3,5 e 6 ).

Esta análise Bayesiana é realizada a partir de tres motivações:

1- A maioria dos projetos de engenharia de materiais são processos evolucionários, devido a grandes modificações e inovações necessárias para se adequar as novas exigências na área industrial. Assim a informação de projetos anteriores pode ser utilizada como uma tentativa para melhorar os resultados de um novo projeto, isto é, utilizando informação a priori. Portanto, esta consideração conduz a utilização de técnicas Bayesianas.

2- Análise adequada quando existem restrições de recursos experimentais tais como:

a) Tempo de teste.

b) Tamanho de amostras pequeno.

c) Procedimento de teste de custo elevado, principalmente quando se trata de um material que requer uma alta confiabilidade.

3- Os cálculos dos estimadores de máxima verossimilhança para os parâmetros são matematicamente intratáveis, isto é, devemos usar métodos iterativos. Com a utilização de métodos Bayesianos simplificamos estes cálculos, pois são encontradas fórmulas simples para as densidades a posteriori marginais e densidades preditivas.

Embora o objetivo principal deste trabalho seja uma análise Bayesiana, apresentaremos inicialmente uma análise clássica para o modelo da distribuição de BirnbaumSaunders (sem testes acelerados) e para o modelo Log-Linear da distribuição de BirnbaumSaunders (com testes acelerados) considerando uma variável Stress, ver capítulos $2 \mathrm{e}$ 4 , respectivamente. A seguir desenvolvemos uma análise Bayesiana considerando priori 
não-informativas (para o modelo da distribuição de Birnbaum-Saunders e para o modelo Log-Linear da distribuição de Birnbaum-Saunders) e encontramos fórmulas simples das posteriori de interesse utilizando o método de aproximação de LAPLACE para integrais. Apesar dos resultados obtidos considerarem priori não-informativas, podemos obter de forma análoga, resultados assumindo priori informativas, como mostramos no capítulo 6 para o modelo Log-Linear da distribuição de Birnbaum-Saunders.

Este trabalho destina-se ao estudo de ruptura de materiais causadas por experimentos de fadiga. Como todo experimento deve ser cuidadosamente planejado para minimizar o tempo e custo dos ensaios, apresentamos um estudo sobre planejamento de experimentos de dois fatores, sob o modelo Log-Linear da distribuição de BirnbaumSaunders, considerando o enfoque clássico e Bayesiano (ver capítulo 7).

Três exemplos de aplicação são desenvolvidos no capítulo 8 , empregando as metodologias obtidas (sem testes acelerados, e com testes acelerados). Também fornecemos algumas direções para futuros trabalhos, relacionados com a distribuição de Birnbaum-Saunders. 


\section{Capitulo 2}

\section{Análise Clássica do Modelo de Birnbaum-Saunders}

\subsection{Estimadores de máxima verossimilhança para os parâmetros $\alpha$ e $\beta$.}

Seja $t_{1}, \ldots, t_{n}$ uma amostra aleatória de tamanho $n$ de uma população com distribuição de Birnbaum-Saunders com função densidade de probabilidade (f.d.p.) dada por (1.13). Geralmente nosso objetivo é obter informações a respeito dos parâmetros, isto é obter informações sobre características numéricas desconhecidas da população. Um estimador muito utilizado devido a suas boas propriedades é dado pelo estimador de máxima verossimilhança. Para escrever a função de verossimilhança (ver, Engelhardt, Bain e Wright, 1981) para $\alpha$ e $\beta$, considerar a notação,

$$
S=\frac{1}{n} \sum_{i=1}^{n} t_{i} \quad e \quad R=\left[\frac{1}{n} \sum_{i=1}^{n} t_{i}^{-1}\right]^{-1}
$$

onde $\mathrm{S}$ e $\mathrm{R}$ representam a média aritmética e harmônica respectivamente. Isto é,

$$
n S=\sum_{i=1}^{n} t_{i} \quad \text { e } \quad n R^{-1}=\sum_{i=1}^{n} \frac{1}{t_{i}} .
$$


A função de verossimilhança para $\alpha$ 'e $\beta$ é dada por:

$$
L(\alpha, \beta)=\left[\frac{1}{2 \sqrt{2 \pi}}\right]^{n}\left[\frac{1}{\alpha \beta^{1 / 2}}\right]^{n} \prod_{i=1}^{n}\left[\frac{t_{i}+\beta}{t_{i}^{3 / 2}}\right] \exp \left[-\frac{n S}{2 \beta \alpha^{2}}-\frac{n \beta}{2 R \alpha^{2}}+\frac{n}{\alpha^{2}}\right]
$$

Geralmente é mais fácil trabalhar com o logaritmo da função de verossimilhança, para se obter o seu máximo, pois os valores de $\alpha$ e $\beta$ que maximizam o logaritmo da função de máxima verossimilhança de igual maneira maximizam a função de verossimilhança. O logaritmo da função de verossimilhança (2.3) para $\alpha$ e $\beta$ (da parte informativa) é dado por,

$$
l(\alpha, \beta)=-n \ln \alpha-\frac{n}{2} \ln \beta+\sum_{i=1}^{n} \ln \left(t_{i}+\beta\right)-\frac{n S}{2 \beta \alpha^{2}}-\frac{n \beta}{2 R \alpha^{2}}+\frac{n}{\alpha^{2}} .
$$

As primeiras derivadas de (2.4) em relação a $\alpha$ e $\beta$ são, dadas por,

$$
\frac{\partial l(\alpha, \beta)}{\partial \alpha}=-\frac{n}{\alpha}+\frac{n S}{\alpha^{3} \beta}+\frac{n \beta}{R \alpha^{3}}-\frac{2 n}{\alpha^{3}}
$$

e

$$
\frac{\partial l(\alpha, \beta)}{\partial \beta}=-\frac{n}{2 \beta}+\sum_{i=1}^{n} \frac{1}{\left(t_{i}+\beta\right)}+\frac{n S}{2 \alpha^{2} \beta^{2}}-\frac{n}{2 R \alpha^{2}}
$$

respectivamente.

Portanto igualando a zero a expressão (2.5) e resolvendo esta equação, o estimador de máxima verossimilhança de $\alpha$ em funçâo de $\hat{\beta}$ é dado por:

$$
\widehat{\alpha}=\left(\frac{S}{\widehat{\beta}}+\frac{\widehat{\beta}}{R}-2\right)^{1 / 2}
$$

De igual forma igualando (2.6) a zero temos,

$$
-\frac{n}{2 \widehat{\beta}}+n\left(K^{*}\right)^{-1}(\widehat{\beta})+\frac{n S}{2 \widehat{\alpha}^{2} \widehat{\beta}^{2}}-\frac{2}{2 R \widehat{\alpha}^{2}}=0
$$

com $R<\widehat{\beta}<S$, onde R e S são dadas por (2.1) e,

$$
K^{*}(\hat{\beta})=\left[\frac{1}{n} \sum_{i=1}^{n}\left(\dot{t}_{i}+\widehat{\beta}\right)^{-1}\right]^{-1}
$$


(ver, Engelhardt, Bain e Wrigt, 1981) é a função média harmônica, isto é,

$$
n\left(K^{*}\right)^{-1}(\widehat{\beta})=\sum_{i=1}^{n}\left(t_{i}+\widehat{\beta}\right)^{-1} .
$$

Assim substituíndo (2.7) em (2.8) e resolvendo obtemos a função aleatória $G(\widehat{\beta})$ definida por,

$$
G(\widehat{\beta})=\widehat{\beta}^{2}-\widehat{\beta}\left[2 R+K^{*}(\widehat{\beta})\right]+R\left[S+K^{*}(\widehat{\beta})\right],
$$

$\operatorname{com} R<\widehat{\beta}<S$.

Portanto, o estimador de máxima verossimilhança para $\beta$ é a única solução positiva da equação $G(\widehat{\beta})=0$. Para resolver $G(\widehat{\beta})=0$ devemos usar métodos iterativos, para encontrar uma solução numérica (ver, Birnbaum e Saunders, 1969-b).

\subsection{Inferências sobre os parâmetros $\alpha$ e $\beta$.}

Para n. grande é usual fazer inferências sobre os parâmetros $\alpha$ e $\beta$, usando a aproximação Normal assintótica dos estimadores de máxima verossimilhança $\hat{\alpha}$ e $\widehat{\beta}$ (ver por exemplo, Mood, Graybill e Boes, 1974). Assim consideramos,

$$
(\widehat{\alpha}, \widehat{\beta}) \stackrel{a}{\sim} N\left\{(\alpha, \beta) ; I^{-1}(\widehat{\alpha}, \widehat{\beta})\right\},
$$

onde $I(\alpha, \beta)$ é a Matriz de Informação de Fisher, dada por:

$$
I(\alpha, \beta)=\left(\begin{array}{lr}
E\left\{-\partial^{2} l(\alpha, \beta) / \partial \alpha^{2}\right\} & E\left\{-\partial^{2} l(\alpha, \beta) / \partial \alpha \partial \beta\right\} \\
E\left\{-\partial^{2} l(\alpha, \beta) / \partial \beta \partial \alpha\right\} & E\left\{-\partial^{2} l(\alpha, \beta) / \partial \beta^{2}\right\}
\end{array}\right),
$$

a qual é obtida das esperanças das segundas derivadas parciais de $l(\alpha, \beta)$ com respeito a $\alpha$ e $\beta$ a menos do sinal.

As segundas derivadas parciais de (2.4) com respeito a $\alpha$ e $\beta$ são dadas por,

$$
\frac{\partial^{2} l(\alpha, \beta)}{\partial \alpha^{2}}=\frac{n}{\alpha^{2}}+\frac{3 n}{\alpha^{4}}\left[-\frac{S}{\beta}-\frac{\beta}{R}+2\right]
$$




$$
\begin{gathered}
\frac{\partial^{2} l(\alpha, \beta)}{\partial \alpha \partial \beta}=\frac{n}{\alpha^{3}}\left[-\frac{S}{\beta^{2}}+\frac{1}{R}\right] \\
\frac{\partial^{2} l(\alpha, \beta)}{\partial \beta^{2}}=-\frac{n S}{\alpha^{2} \beta^{3}}-\sum_{i=1}^{n}\left(t_{i}+\beta\right)^{-2}+\frac{n}{2 \beta^{2}}
\end{gathered}
$$

Para calcular os elementos da matriz de informação de Fisher observe que,

$$
E[n S]=n \beta\left[1+\frac{\alpha^{2}}{2}\right]
$$

(ver a fórmula para $\mathrm{E}[\mathrm{T}]$ dada em (1.15)).

Por outro lado, por (1.12) Z é normalmente distribuida com média zero e variancia 1. Portanto, $Z^{2}$ tem uma distribuição Qui-Quadrado com 1 grau de liberdade, a qual tem média 1 e variancia 2 . Assim,

$$
E\left[\frac{1}{T}\right]=\frac{1}{\beta}\left[\frac{\alpha^{2}}{2}+1\right],
$$

o que implica que,

$$
E\left[n R^{-1}\right]=\sum_{i=1}^{n} E\left[\frac{1}{t}\right]=\frac{n}{\beta}\left[\frac{\alpha^{2}}{2}+1\right]
$$

Substituindo as expressões (2.15) e (2.16) no valor esperado de (2.12), temos,

$$
E\left\{-\frac{\partial^{2} l(\alpha, \beta)}{\partial \alpha^{2}}\right\}=\frac{2 n}{\alpha^{2}}
$$

Da mesma forma, de (2.13), temos,

$$
E\left\{-\frac{\partial^{2} l(\alpha, \beta)}{\partial \alpha \partial \beta}\right\}=E\left\{-\frac{\partial^{2} l(\alpha, \beta)}{\partial \beta \partial \alpha}\right\}=0
$$

Observar que, como

$$
E\left\{-\frac{\partial^{2} l(\alpha, \beta)}{\partial \alpha \partial \beta}\right\}=0
$$


o parâmetro $\alpha$ é ortogional á $\beta$.

Finalmente substituindo a expressão (2.15) no valor esperado de (2.14), temos,

$$
E\left[-\frac{\partial^{2} l(\alpha, \beta)}{\partial \beta^{2}}\right]=n\left[E\left(\frac{1}{t+\beta}\right)^{2}+\frac{1}{\alpha^{2} \beta^{2}}\right]
$$

Portanto, a Matriz de Informação de Fisher para $\alpha$ e $\beta$ é dada por:

$$
I(\alpha, \beta)=\left[\begin{array}{lc}
\frac{2 n}{\alpha^{2}} & 0 \\
0 & n\left[E\left(\frac{1}{t+\beta}\right)^{2}+\frac{1}{\alpha^{2} \beta^{2}}\right]
\end{array}\right]
$$

onde

$$
E\left[\frac{1}{T+\beta}\right]^{2}=\frac{1}{2 \sqrt{2 \pi} \alpha \beta^{1 / 2}} \int_{0}^{\infty} \frac{1}{t^{3 / 2}(t+\beta)} \exp \left\{\frac{-1}{2 \alpha^{2}}\left[\frac{t}{\beta}+\frac{\beta}{t}-2\right]\right\} d t,
$$

a qual é calculada por integração numérica.

Observamos que, a integral dada em (2.18) também pode ser calculada aproximadamente, utilizando o método de LAPLACE (ver por exemplo, Kass, Tierney e Kadane, 1990). Escrevendo (2.18) na forma:

$$
E\left[\frac{1}{T+\beta}\right]^{2}=\frac{1}{2 \sqrt{2 \pi} \alpha \beta^{1 / 2}} \int_{0}^{\infty} f(t) \exp [-n h(t)] d t
$$

onde,

$$
f(t)=\frac{1}{t^{3 / 2}(t+\beta)}
$$

e

$$
-n h(t)=\frac{-1}{2 \alpha^{2}}\left[\frac{t}{\beta}+\frac{\beta}{t}-2\right]
$$

Portanto usando a aproximação de LẢPLACE (ver, apêndice A) , temos,

$$
E\left(\frac{1}{T+\beta}\right)^{2} \simeq \frac{1}{4 \beta^{2}}
$$


Assim, da aproximação de LAPLACE, obtemos uma forma simplificada da matriz de informação de Fisher para $\alpha$ e $\beta$, dada por,

$$
I(\alpha, \beta)=\left[\begin{array}{cc}
\frac{2 n}{\alpha^{2}} & 0 \\
0 & \frac{n}{\beta^{2}}\left[\frac{1}{\alpha^{2}}+\frac{1}{4}\right]
\end{array}\right]
$$

Utilizando a distribuição normal assintótica dos estimadores de máxima verossimilhança $\widehat{\alpha}$ e $\widehat{\beta}$, a qual é dada por (2.11), com $I(\alpha, \beta)$ dada por (2.21), é possível calcular por meio de expressões simples a variancia assintótica (estimada) dos estimadores de máxima verossimilhança $\widehat{\alpha}$ e $\widehat{\beta}$, dadas por,

$$
\operatorname{vâ} r(\hat{\alpha}) \simeq \frac{\hat{\alpha}^{2}}{2 n} \quad e \cdot \operatorname{va} r(\widehat{\beta}) \simeq \frac{\widehat{\beta}^{2}}{n}\left(\frac{1}{\widehat{\alpha}^{2}}+\frac{1}{4}\right)^{-1},
$$

respectivamente.

Portanto, os intervalos de confiança para os parâmetros $\alpha$ e $\beta$, considerando um nível de confiança $100(1-\phi) \%$, são fornecidos por:

$$
\begin{aligned}
I C(\alpha) & =\left[\widehat{\alpha} \pm Z_{\phi / 2} \sqrt{v \hat{a} r(\hat{\alpha})}\right], \\
I C(\beta) & =\left[\hat{\beta} \pm Z_{\phi / 2} \sqrt{v \widehat{a} r(\hat{\beta})}\right],
\end{aligned}
$$

onde $Z_{\phi / 2}$ é um percentil da distribuição Normal padronizada (ver por exemplo, Kalfleisch, $1985)$.

Usualmente, industrias que trabalham com dadọs de fadiga têm interesse em inferências sobre o tempo médio de sobrevivência ou tempo médio da fadiga, o qual usando (1.15) é dado por

$$
\lambda^{*}=E[T]=\beta\left(1+\frac{\alpha^{2}}{2}\right) .
$$

Para fazer inferências sobre $\lambda^{*}$, podemos usar o método DELTA (ver por exemplo, Miller, 1981). Considerando a matriz de informação de Fisher em sua forma simplificada $(2.21)$, o estimador de máxima verossimilhança $\widehat{\lambda^{*}}=\widehat{\beta}\left(1+\frac{\widehat{\alpha}^{2}}{2}\right)$ tem uma distribuição normal assintótica dada por,

$$
\widehat{\lambda^{*}} \stackrel{a}{\sim} N\left[\omega ; \sigma_{0}^{2}\right]
$$


onde $\lambda^{*}$ é dado por (2.25), e

$$
\sigma_{0}^{2}=\frac{\hat{\beta}^{2}}{n}\left\{\frac{\hat{\alpha}^{4}}{2}+\left(1+\frac{\hat{\alpha}^{2}}{2}\right)^{2}\left(\frac{1}{4}+\frac{1}{\hat{\alpha}^{2}}\right)^{-1}\right\}
$$




\section{Capítulo 3}

\section{Análise Bayesiana do Modelo de Birnbaum-Saunders}

\subsection{Análise Bayesiana assumindo $\alpha$ e $\beta$ desconheci- dos.}

\subsubsection{Densidades a priori não-informativas para $\alpha$ e $\beta$.}

Depois de ter realizado uma análise clássica do modelo de Birnbaum-Saunders, realizaremos uma análise Bayesiana, considerando duas densidades a priori não-informativas para representar o grau de conhecimento sobre os parâmetros $\alpha$ e $\beta$. Para tal propósito, primeiramente consideramos a densidadé a priori não-informativa de Jeffreys (ver por exemplo, Box e Tiao, 1973), dada por,

$$
P(\alpha, \beta) . \propto|\operatorname{det} I(\alpha, \beta)|^{1 / 2},
$$

onde $I(\alpha, \beta)$ é a matriz de informação de Fisher para $\alpha$ e $\beta(2.21)$, isto é a matriz de informação de Fisher em sua forma simplificada. Por conseguinte uma densidade a priori não informativa para $\alpha$ e $\beta$, por (3.1) é dada por,

$$
P(\alpha, \beta) \propto \frac{1}{\alpha \beta}\left(\frac{1}{\dot{\alpha}^{2}}+\frac{1}{4}\right)^{1 / 2},
$$

onde $\alpha, \beta>0$. 
Observar que $\alpha$ é ortogonal a $\beta$ (pois

$$
\left.E\left\{-\frac{\partial^{2} l(\alpha, \beta)}{\partial \alpha \partial \beta}\right\}=0\right)
$$

Isto leva a outra densidade a priori não-informativa para $\alpha$ e $\beta$ (ver 'Tibshirani, 1989) dada por,

$$
P(\alpha, \beta) \propto\left(I_{\alpha, \alpha}(\alpha, \beta)\right)^{1 / 2} \times F(\beta),
$$

onde $I_{\alpha, \alpha}(\alpha, \beta)$ é o elemento da matriz de informação (2.21) do parâmetro de interesse $\alpha$ $\left(\left(I_{\alpha, \alpha}(\alpha, \beta)\right)^{1 / 2} \propto \frac{1}{\alpha}\right)$, e $F(\beta)$ é uma função arbitraria positiva do parâmetro perturbador $\beta\left(F(\beta) \propto \frac{1}{\beta}\right)$. Portanto uma densidade a priori não-informativa para $\alpha$ e $\beta$ por (3.3) é, dada por,

$$
P(\alpha, \beta) \propto \frac{1}{\alpha \beta},
$$

para $\alpha, \beta>0$, que coincide com a densidade a priori não-informativa para $\alpha$ e $\beta$ assumindo independência entre $\alpha$ e $\beta$ (ver por exemplo, Box e Tiao, 1973).

\subsubsection{Densidade a posteriori conjunta para $\alpha$ e $\beta$.}

Combinando a função de verossimilhança (2.3) com as densidades a priori (3.2) e (3.4), a densidade a posteriori conjunta para $\alpha$ e $\beta$ (ver por exemplo, Zellner, 1971) é dada por,

$$
P(\alpha, \beta / D a d o s) \propto \frac{\prod_{i=1}^{n}\left(t_{i}+\beta\right) \exp \left\{\frac{-A(\beta)}{\alpha^{2}}\right\}}{\alpha^{n+1} \beta^{n / 2+1} H\left(\alpha^{2}\right)},
$$

$\operatorname{para} \alpha, \beta>0$ e

$$
A(\beta)=\frac{n S}{2 \beta}+\frac{n \beta}{2 R}-n
$$

com

$$
H\left(\alpha^{2}\right)=\left(\frac{1}{\alpha^{2}}+\frac{1}{4}\right)^{-1 / 2}
$$

se a densidade a priori for de Jeffreys, e $H\left(\alpha^{2}\right)=1$, se a priori for de TIBSHIRANI. 


\subsubsection{Densidades a posteriori marginais para $\alpha$ e $\beta$.}

Em inferência Bayesiana, inferências são tipicamente baseadas nas densidades a posteriori marginais dos parâmetros considerados, em nosso caso $\alpha$ e $\beta$. As densidades a posteriori marginais para $\alpha$ e $\beta$ são obtidas por integração da densidade a posteriori conjunta (3.5) em $\alpha$ ou $\beta$, respectivamente. Como estas integrais, em geral, não apresentam soluçóes analíticas explícitas, utilizaremos frequentemente o método de LAPLACE para aproximaçâo de integrais (ver por exemplo, Kass, Tierney e Kadane, 1990).

A densidade a posteriori marginal para $\alpha$ considerando a densidade a priori não-informativa (3.2), pode ser escrita da seguinte forma:

$$
P(\alpha / D a d o s) \propto e^{n / \alpha^{2}} \alpha^{-(n+2)}\left(4+\alpha^{2}\right)^{1 / 2} \int_{0}^{\infty} f(\beta) \exp [-n h(\beta)] d \beta,
$$

onde,

$$
f(\beta)=\frac{\prod_{i=1}^{n}\left(t_{i}+\beta\right)}{\beta^{n / 2+1}}
$$

e

$$
-n h(\beta)=-\frac{n S}{2 \beta \alpha^{2}}-\frac{n \beta}{2 R \alpha^{2}} .
$$

Utilizando a aproximação de LAPLACE em (3.6) (ver, apêndice A) obtemos a densidade a posteriori marginal aproximada para $\alpha$ dada por,

$$
P(\alpha / D a d o s) \propto\left(4+\alpha^{2}\right)^{1 / 2} \alpha^{-(n+1)} \exp \left\{-\frac{n}{\alpha^{2}}\left[\left(\frac{S}{R}\right)^{1 / 2}-1\right]\right\},
$$

para $\alpha>0$.

Similarmente, considerando a densidade a priori não-informativa (3.4), e usando aproximação de LAPLACE, a densidade a posteriori marginal aproximada para $\alpha$ é dada por,

$$
P(\alpha / D a d o s) \propto \alpha^{-n} \exp \left\{-\frac{n}{\alpha^{2}}\left[\left(\frac{S}{R}\right)^{1 / 2}-1\right]\right\},
$$

para $\alpha>0$. 
A densidade a posteriori marginal para $\beta$ considerando a densidade a priori não informativa (3.2), pode ser expressa da seguinte maneira:

$$
P(\beta / D a d o s) \propto \frac{\prod_{i=1}^{n}\left(t_{i}+\beta\right)}{\beta^{n / 2+1}} \int_{0}^{\infty} f(\alpha) \exp [-n h(\alpha)] \cdot d \alpha,
$$

onde,

$$
f(\alpha)=\left(4+\alpha^{2}\right)^{1 / 2},
$$

e

$$
-n h(\alpha)=-\frac{A(\beta)}{\alpha^{2}}-(n+2) \ln \alpha .
$$

Novamente, utilizando a aproximação de LAPIACE em (3.9), temos que a densidade a posteriori marginal aproximada para $\beta$ é dada por,

$$
P(\beta / D a d o s) \propto \frac{\prod_{i=1}^{n}\left(t_{i}+\beta\right)\left[4+\frac{2 n}{(n+2)}\left(\frac{S}{2 \beta}+\frac{\beta}{2 R}-1\right)\right]^{1 / 2}}{\beta^{n / 2+1}\left[\frac{S}{2 \beta}+\frac{\beta}{2 R}-1\right]^{(n+1) / 2}},
$$

para $\beta>0$.

Por outro lado, a densidade a posteriori marginal para $\beta$ considerando a densidade a priori não-informativa (3.4), pode ser escrita da seguinte forma,

$$
P(\beta / D a d o s) \propto \frac{\prod_{i=1}^{n}\left(t_{i}+\beta\right)}{\beta^{n / 2+1}} \int_{0}^{\infty} \alpha^{-(n+1)} \exp \left[-A(\beta) \alpha^{-2}\right] d \alpha .
$$

Resultado (ver por exemplo, Box e Tiao, 1973): .

\section{Resultado 3.1}

$$
\int_{0}^{\infty} x^{-(p+1)} e^{-A x^{-2}} d x=\frac{1}{2} A^{-p / 2} \Gamma\left(\frac{p}{2}\right) .
$$

Usando o resultado anterior, a integral dada em (3.11) é facilmente calculada, na forma exata. Assim a densidade a posteriori marginal para $\beta$ é dada por,

$$
P(\beta / D a d o s) \propto \frac{\beta^{-(n / 2+1)} \prod_{i=1}^{n}\left(t_{i}+\beta\right)}{\left[\frac{S}{2 \beta}+\frac{\beta}{2 R}-1\right]^{n / 2}},
$$

para $\beta>0$ 


\subsection{Análise Bayesiana ássumindo $\beta$ conhecido.}

Assumindo $\beta$ conhecido, de (2.3), obtemos a função de verossimilhança para $\alpha$, dada por,

$$
L(\alpha) \propto \alpha^{-n} \exp \left[-\frac{A(\beta)}{\alpha^{2}}\right] .
$$

Consideramos uma densidade a priori não-informativa de Jeffreys para $\alpha$, dada por,

$$
P(\alpha) \propto \frac{1}{\alpha}
$$

onde $\alpha>0$.

Portanto, de (3.14) e (3.15), e utilizando o resultado (3.1), a densidade a posteriori para $\alpha$ é dada por,

$$
P(\alpha / \text { Dados })=\frac{2[A(\beta)]^{n / 2} \alpha^{-(n+1)}}{\Gamma(n / 2)} \exp \left[-\frac{A(\beta)}{\alpha^{2}}\right]
$$

para $\alpha>0$

Como vimos no capítulo 2 , as industrias de materiais que trabalham com fadigas, usualmente tem interesse em inferir sobre o tempo médio de sobrevivência ou tempo médio de vida da fadiga.

Por conseguinte, considerar a transformação de variáveis dada por

$$
\xi=\beta\left(1+\frac{\alpha^{2}}{2}\right)
$$

(ver $(2.25))$. Então

$$
\alpha=\left[\frac{2(\xi-\beta)}{\beta}\right]^{1 / 2} \quad \text { e } \quad\left|\frac{\partial \alpha}{\partial \xi}\right|=\frac{1}{[2 \beta(\xi-\beta)]^{1 / 2}}
$$

onde $\frac{\partial \alpha}{\partial \xi}$ é o Jacobiano da transformação. Assim, de (3.16) e (3.17) a densidade a posteriori para o tempo médio de vida da fadiga

$$
\xi=\beta\left(1+\frac{\alpha^{2}}{2}\right)
$$


é dada por,

$$
P(\xi / \text { Dados })=\frac{[A(\beta)]^{n / 2} \beta^{n / 2}}{2^{n / 2} \Gamma\left(\frac{n}{2}\right)(\xi-\beta)^{n / 2+1}} \exp \left[-\frac{\beta A(\beta)}{2(\xi-\beta)}\right]
$$

$\operatorname{para} \xi>0$

A moda da densidade a posteriori (3.18) é dada por,

$$
\widehat{\xi}^{*}=\beta+\frac{\beta}{(n+2)} A(\beta) .
$$

Para calcular a moda basta derivar o logaritmo de (3.18) em relação a $\xi$, igualar a zero e resolver, em $\xi$.

De (3.18), observarnos que

$$
(\beta /(\xi-\beta)) A(\beta)
$$

dado os dados, tem uma distribuição Qui-Quadrado com n graus de liberdade. Portanto, um intervalo de credibilidade para $\xi$ com probabilidade $1-\phi$ é dado por,

$$
I C(\xi)=\left[\beta\left(1+\frac{A(\beta)}{\chi_{n(1-\phi / 2)}^{2}}\right) ; \beta\left(1+\frac{A(\beta)}{\chi_{n(\phi / 2)}^{2}}\right)\right]
$$

onde $\chi_{n(\phi / 2)}^{2}$ é um percentil da distribuição Qui-Quadrado com n graus de liberdade, tal que,

$$
P\left(\chi_{n}^{2} \leq \chi_{n(\phi / 2)}^{2}\right)=\frac{\phi}{2}
$$

\subsection{Densidade preditiva para uma observação fu- tura}

Assumindo $\beta$ conhecido e a densidade a priori não- informativa de Jeffreys para $\alpha(3.15)$, a densidade preditiva para uma observação futurâ $t_{(n+1)}$ (ver por exemplo, Press, 1989) é dada por,

$$
f\left(t_{(n+1)} / \text { Dados }\right)=\int_{0}^{\infty} f\left(t_{(n+1)} / \alpha\right) P(\alpha / D a d o s) d \alpha
$$


onde $P(\alpha /$ Dados $)$ é dada por (3.16), e

$$
f\left(t_{(n+1)} / \alpha\right)=\frac{\left(\beta+t_{(n+1)}\right)}{2 \sqrt{2 \pi} \alpha \beta^{1 / 2} t_{(n+1)}^{3 / 2}} \exp \left\{-\frac{1}{\alpha^{2}}\left[\frac{t_{(n+1)}}{2 \beta}+\frac{\beta}{2 t_{(n+1)}}-1\right]\right\} .
$$

Portanto usando o resuitado (3.1) a densidade preditiva para uma observação futura $t_{(n+1)}$ é dada por,

$$
f\left(t_{(n+1)} / \text { Dados }\right)=\frac{\left(\beta+t_{(n+1)}\right)(A(\beta))^{n / 2} \Gamma\left(\frac{n+1}{2}\right)}{2 \sqrt{2 \pi} \beta^{1 / 2} t_{(n+1)}^{3 / 2} \Gamma\left(\frac{n}{2}\right)\left(\frac{n+t_{(n+1)}}{2 \beta}+\frac{\beta}{2}\left[\frac{n}{R}+\frac{1}{t_{(n+1)}}\right]-(n+1)\right)^{(n+1) / 2}} .
$$

para $t_{(n+1)}>0$.

O objetivo da expressão (3.23) é fazer uma previsão para a obervação futura $T_{(n+1)}$, isto é, prever $t_{(n+1)}$ a partir da informação amostral (Dados).

Se $\beta$ é desconhecido, podemos usar o método de LAPLACE para encontrar uma densidade preditiva aproximada para uma observação futura $t_{(n+1)}$. 


\section{Capitulo 4}

\section{Análise clássica do modelo $\mathbb{L o g}$-Linear da distribuição de Birnbaum-Saunders}

\subsection{Estimadores de máxima verossimilhança para os parâmetros $\alpha$ e $\theta$.}

O modelo Log-Linear (1.19), pode ser generalizado para um número arbitrário de variáveis explanatórias.

Sejam $T_{1}, \ldots T_{n} \mathrm{n}$ variáveis aleatórias independentes com distribuição de Birnbaum-Saunders, com parâmetros de forma $\alpha_{i}$ e parâmetros de escala $\beta_{i}$. Supondo que $T_{i}$ tem distribuição dependente de $\mathrm{p}$ variáveis explanatórias, denotadas por:

$$
\underline{X}_{i}=\left(X_{i 1}, \ldots X_{i p}\right)
$$

então temos as seguintes suposições,

Suposição $4.1 \beta_{i}=\exp \left[\underline{X}_{i}^{\prime} \underline{\Theta}\right]$, para $i=1,2, \ldots n$ onde $\underline{\Theta}^{\prime}=\left(\theta_{1}, \ldots \theta_{p}\right)$ é um vetor de parâmetros desconhecidos o qual deve ser estimado.

Suposição 4.2 $O$ parâmetro de forma é independente do vetor explanatório $\underline{X}_{\mathrm{i}}$, isto é, $\alpha_{i}=\alpha$ para $i=1,2, \ldots n$. 
Birnbaum e Saunders (1969-b) mostram que se $K>0$, então $K T_{i}$ tem uma distribuiçâao de Birnbaum-Saunders com parâmetro de forma $\alpha$ e parâmetro de escala $K \beta_{i}$. Usando este fato e as suposições (4.1) e (4.2) antes mencionadas, vemos que $T_{i}$ pode ser expressa como:

$$
T_{i}=\exp \left[\underline{X}_{i} \Theta\right] \delta_{i}
$$

onde os $\delta_{i}$ tem distribuição de Birnbaum-Saunders com parâmetros de forma $\alpha$ e parâmetro de escala 1. Portanto aplicando logaritmo em (4.2), temos,

$$
Y_{i}=\ln \left(T_{i}\right)=\underline{X}_{i} \underline{\Theta}+\varepsilon_{i}
$$

que é o modelo Log-Linear em sua forma generalizada, onde $\varepsilon_{i}=\ln \left(\delta_{i}\right)$ é o erro do modelo, o qual tem uma distribuição Senh-Normal com parâmetros $\alpha, \gamma=0$ e $v=2$.

Um caso especial do modelo (4.3), para uma variável Stress $x_{i}$, para $i=$ $1,2, \ldots n$ é dado por:

$$
Y_{i}=a+b x_{i}+\varepsilon_{i}
$$

para $i=1,2, \ldots n, \operatorname{com} \underline{\Theta}^{\prime}=(a, b)$ e $\varepsilon_{i} \sim S N(\alpha, 0,2)$.

Sejam $Y_{1}, \ldots Y_{n}$ n observações independentes do modelo (4.4), $\operatorname{com} \varepsilon_{i}=y_{i}-$ $a-b x_{i}$ onde $\varepsilon_{i} \sim S N(\alpha, 0,2)$. A função densidade de probabilidade de $\varepsilon_{i}$ é dada na forma,

$$
f(\varepsilon)=\left(\frac{2}{2 \sqrt{2 \pi} \alpha}\right) \cosh \left[\frac{y_{i}-a-b x_{i}}{2}\right] \exp \left\{-\frac{2}{\alpha^{2}} \operatorname{senh}^{2}\left[\frac{y_{i}-a-b x_{i}}{2}\right]\right\}
$$

para $-\infty<a, b<\infty, \alpha>0$ e $-\infty<\varepsilon<\infty$.

Portanto a função de verossimilhança para a,b e $\alpha$ é dada por,

$$
L(a, b, \alpha)=\left(\frac{1}{2 \sqrt{2 \pi}}\right)^{n} \prod_{i=1}^{n} W_{i} \exp \left\{-\frac{1}{2} \sum_{i=1}^{n} Z_{i}^{2}\right\}
$$

Por razões explicadas no capítulo 2 , é recomendavel trabalhar com o logaritmo da função de verossimilhança. Assim o logaritmo da função de verossimilhança (da parte informativa) para a,b e $\alpha$ é,

$$
l(a, b, \alpha)=\sum_{i}^{n} \ln W_{i}-\frac{1}{2} \sum_{i}^{n} Z_{i}^{2}
$$


onde

$$
W_{i}=\frac{2}{\alpha} \cosh \left[\frac{y_{i}-a-b x_{i}}{2}\right]
$$

e

$$
Z_{i}=\frac{2}{\alpha} \operatorname{senh}\left[\frac{y_{i}-a-b x_{i}}{2}\right]
$$

Derivando (4.7) em relação a a,b e $\alpha$, obtemos as seguintes equações de verossimilhança:

$$
\begin{gathered}
\frac{\partial l(a, b, \alpha)}{\partial a}=\frac{1}{2} \sum_{i=1}^{n}\left\{Z_{i} W_{i}-\frac{Z_{i}}{W_{i}}\right\}=0 \\
\frac{\partial l(a, b, \alpha)}{\partial b}=\frac{1}{2} \sum_{i=1}^{n} x_{i}\left\{Z_{i} W_{i}-\frac{Z_{i}}{W_{i}}\right\}=0
\end{gathered}
$$

e,

$$
\frac{\partial l(a, b, \alpha)}{\partial \alpha}=-\frac{n}{\alpha}+\frac{1}{\alpha} \sum_{i=1}^{n} Z_{i}^{2}=0 .
$$

Portanto resolvendo a equação (4.10) encontramos uma expressão para o estimador de máxima verossimilhança de $\alpha^{2}$ em termos de $\widehat{a}$ e $\widehat{b}$, a qual é dada por :

$$
\widehat{\alpha}^{2}=\frac{4}{n} \sum_{i=1}^{n} \operatorname{senh}^{2}\left[\frac{y_{i}-\hat{a}-\hat{b} x_{i}}{2}\right],
$$

onde $\widehat{a}$ e $\widehat{b}$ são os estimadores de máxima verossimilhança de $a$ e $b$, os quais são obtidos numericamente.

\subsection{Inferências sobre os parâmetros $\mathrm{a}, \mathrm{b}$ e $\alpha$.}

Para n grande é usual fazer inferências sobre os parâmetros $\mathrm{a}, \mathrm{b}$ e $\alpha$, utilizando a aproximação Normal assintótica dos estimadores de máxima verossimilhança $\widehat{a}, \hat{b}$ e $\hat{\alpha}$ (ver por exemplo, Mood, Graybill e Boes, 1974). Assim consideramos,

$$
(\widehat{a}, \widehat{b}, \widehat{\alpha}) \stackrel{a}{\sim} N\left\{(a, b, \alpha) ; l_{0}^{-1}\right\}
$$


onde $I_{0}$ é a matriz de informação observada, dada por:

$$
I_{0}=\left(\begin{array}{ccr}
-\frac{1}{4} \widehat{s}_{0} & -\frac{1}{4} \widehat{s}_{1} & \frac{1}{\hat{\alpha}} \sum_{i=1}^{n} \hat{Z}_{i} \widehat{W}_{i} \\
-\frac{1}{4} \widehat{s}_{1} & -\frac{1}{4} \widehat{s}_{2} & \frac{1}{\alpha} \sum_{i=1}^{n} x_{i} \widehat{Z}_{i} \widehat{W}_{i} \\
\frac{1}{\alpha} \sum_{i=1}^{n} \widehat{Z}_{i} \widehat{W}_{i} & \frac{1}{\hat{\alpha}} \sum_{i=1}^{n} x_{i} \widehat{Z}_{i} \widehat{W}_{i} & -\frac{1}{\widehat{\alpha}^{2}}\left(3 \sum_{i=1}^{n} \hat{Z}_{i}^{2}-n\right)
\end{array}\right),
$$

onde

$$
\begin{aligned}
& \widehat{W}_{i}=\frac{2}{\hat{\alpha}} \cosh \left[\frac{y_{i}-\widehat{a}-\hat{b} x_{i}}{2}\right], \\
& \hat{Z}_{i}=\frac{2}{\hat{\alpha}} \operatorname{senh}\left[\frac{y_{i}-\widehat{a}-\hat{b} x_{i}}{2}\right],
\end{aligned}
$$

e

$$
\widehat{s}_{l}=\sum_{i=1}^{n} x_{i}^{l}\left(1-\widehat{W}_{i}^{2}-\widehat{Z}_{i}^{2}-\frac{\hat{Z}_{i}^{2}}{\widehat{W}_{i}^{2}}\right)
$$

para $l=0,1,2$. Onde os elementos de (4.13) são as segundas derivadas parciais estimadas de $l(a, b, \alpha)$ em relação a a, b e $\alpha$ a menos do sinal.

Também podemos utilizar a matriz de informação de Fisher $I(a, b, \alpha)$ em lugar de $I_{0}$ na expressão (4.12). A Matriz de Informação de Fisher, é dada por:

$$
I(a, b, \alpha)=\left(\begin{array}{ccc}
E\left\{-\frac{\partial^{2} l(a, b, \alpha)}{\partial a^{2}}\right\} & E\left\{-\frac{\partial^{2} l(a, b, \alpha)}{\partial a \partial b}\right\} & E\left\{-\frac{\partial^{2} l(a, b, \alpha)}{\partial a \partial \alpha}\right\} \\
E\left\{-\frac{\partial^{2} l(a, b, \alpha)}{\partial b \partial a}\right\} & E\left\{-\frac{\partial^{2} l(a, b, \alpha)}{\partial b^{2}}\right\} & E\left\{-\frac{\partial^{2} l(a, b, \alpha)}{\partial b \partial \alpha}\right\} \\
E\left\{-\frac{\partial^{2} l(a, b, \alpha)}{\partial \alpha \partial a}\right\} & E\left\{-\frac{\partial^{2} l(a, b, \alpha}{\partial \alpha \partial b}\right\} & E\left\{-\frac{\partial^{2} l(a, b, \alpha)}{\partial \alpha^{2}}\right\}
\end{array}\right),
$$

a qual é obtida das esperanças das segundas derivadas parciais de $l(a, b, \alpha)$ com respeito a a, b e $\alpha$ a menos do sinal.

As segundas derivadas parciais de (4.7) com respeito $a \mathrm{a}, \mathrm{b}$ e $\alpha$ são dadas respectivamente por,

$$
\frac{\partial^{2} l(a, b, \alpha)}{\partial a^{2}}=\frac{1}{4} \sum_{i=1}^{n}\left[1-Z_{i}^{2}-W_{i}^{2}-\frac{Z_{i}^{2}}{W_{i}^{2}}\right],
$$




$$
\begin{gathered}
\frac{\partial^{2} l(a, b ; \alpha)}{\partial a \partial b}=\frac{1}{4} \sum_{i=1}^{n} x_{i}\left[1-Z_{i}^{2}-W_{i}^{2}-\frac{Z_{i}^{2}}{W_{i}^{2}}\right] \\
\frac{\partial^{2} l(a, b, \alpha)}{\partial b^{2}}=\frac{1}{4} \sum_{i=1}^{n} x_{i}^{2}\left[1-Z_{i}^{2}-W_{i}^{2}-\frac{Z_{i}^{2}}{W_{i}^{2}}\right] \\
\frac{\partial^{2} l(a, b, \alpha)}{\partial a \partial \alpha}=-\frac{1}{\alpha} \sum_{i=1}^{n} Z_{i} W_{i} \\
\frac{\partial^{2} l(a, b, \alpha)}{\partial b \partial \alpha}=-\frac{1}{\alpha} \sum_{i=1}^{n} x_{i} Z_{i} W_{i} \\
\frac{\partial^{2} l(a, b, \alpha)}{\partial \alpha^{2}}=\frac{n}{\alpha^{2}}-\frac{3}{\alpha^{2}} \sum_{i=1}^{n} Z_{i}^{2} .
\end{gathered}
$$

Para calcular os elementos da matriz de informação de Fisher observe que,

$$
Z_{i}=\frac{2}{\alpha} \operatorname{senh}\left[\frac{y_{i}-a-b x_{i}}{2}\right] \stackrel{a}{\sim} N(0,1),
$$

isto é $Z_{i}$ é normalmente distribuida com média zero e variancia 1 . Portanto $Z_{i}^{2}$ tem distribuição de Qui-Quadrado com 1 grau de liberdade, a qual tem média, 1 e variancia 2.

Por outro lado por (4.7),

$$
W_{i}=\frac{2}{\alpha} \cosh \left[\frac{y_{i}-a-b x_{i}}{2}\right] .
$$

Assim utilizando o resultado (1.5) do capítulo 1, temos,

$$
W_{i}^{2}=Z_{i}^{2}+\frac{4}{\alpha^{2}} \text {. }
$$

Portanto considerando (4.20) e (4.21) temos que:

$$
\begin{gathered}
E\left\{-\frac{\partial^{2} l(a, b, \alpha)}{\partial a^{2}}\right\}=\frac{1}{4} \sum_{i=1}^{n}\left[1+\frac{4}{\alpha^{2}}+E\left(\frac{Z_{i}^{2}}{Z_{i}^{2}+4 / \alpha^{2}}\right)\right], \\
E\left\{-\frac{\partial^{2} l(a, b, \alpha)}{\partial a \partial b}\right\}=\frac{1}{4} \sum_{i=1}^{n} x_{i}\left[1+\frac{4}{\alpha^{2}}+E\left(\frac{Z_{i}^{2}}{Z_{i}^{2}+4 / \alpha^{2}}\right)\right],
\end{gathered}
$$




$$
\begin{aligned}
E\left\{-\frac{\partial^{2} l(a, b, \alpha)}{\partial b^{2}}\right\} & =\frac{1}{4} \sum_{i=1}^{n} x_{i}^{2}\left[1+\frac{4}{\alpha^{2}}+E\left(\frac{Z_{i}^{2}}{Z_{i}^{2}+4 / \alpha^{2}}\right)\right] \\
& E\left\{-\frac{\partial^{2} l(a, b, \alpha)}{\partial a \partial \alpha}\right\}=0 \\
& E\left\{-\frac{\partial^{2} l(a, b, \alpha)}{\partial b \partial \alpha}\right\}=0 \\
& E\left\{\frac{\partial^{2} l(a, b, \alpha)}{\partial \alpha^{2}}\right\}=\frac{2 n}{\alpha^{2}}
\end{aligned}
$$

Note que, como (4.25) e (4.26) são iguais a zero, $\alpha$ é ortogonal a a e b.

Definindo,

$$
C(\alpha)=1+\frac{4}{\alpha^{2}}+E\left[\frac{Z_{i}^{2}}{Z_{i}^{2}+4 / \alpha^{2}}\right]
$$

em (4.22), (4.23) e (4.24), temos que a matriz de informação de Fisher é dada por:

$$
I(a, b, \alpha)=\left(\begin{array}{lcc}
\frac{n}{4} C(\alpha) & \frac{1}{4} C(\alpha) \sum_{i=1}^{n} x_{i} & 0 \\
\frac{1}{4} C(\alpha) \sum_{i=1}^{n} x_{i} & \frac{1}{4} C(\alpha) \sum_{i=1}^{n} x_{i}^{2} & 0 \\
0 & 0 & \frac{2 n}{\alpha^{2}}
\end{array}\right)
$$

Para valores pequenos de $\alpha(0<\alpha<1)$, podemos considerar,

$$
C(\alpha) \simeq 1+\frac{4}{\alpha^{2}}
$$

(ver Rieck e Nedelman, 1991).

Isto também pode ser visto utilizando o método de LAPLACE; basta observar que:

$$
E\left[\frac{Z_{i}^{2}}{W_{i}^{2}}\right]=\frac{2}{2 \sqrt{2 \pi} \alpha} \int_{-\infty}^{\infty} f\left(y_{i}\right) \exp \left[-n h\left(y_{i}\right)\right] d y_{i}
$$

onde,

$$
f\left(y_{i}\right)=\frac{\operatorname{senh}^{2} \cdot\left[\frac{y_{i}-a-b x_{i}}{2}\right]}{\cosh ^{2}\left[\frac{y_{i}-a-b x_{i}}{2}\right]}
$$




$$
-n h\left(y_{i}\right)=-\frac{2}{\alpha^{2}} \operatorname{senh}^{2}\left[\frac{y_{i}-a-b x_{i}}{2}\right] .
$$

Portanto, considerando a aproximação de LAPLACE (ver, apêndice A), temos

$$
E\left[\frac{Z_{i}^{2}}{W_{i}^{2}}\right] \simeq 0
$$

Assim, para valores pequenos de $\alpha$, obtemos uma forma simplificada da matriz de informação de Fisher para a, b e $\alpha$, dada por:

$$
I(a, b, \alpha)=\left(\begin{array}{lcc}
\frac{n}{4}\left(1+\frac{4}{\alpha^{2}}\right) & \frac{1}{4}\left(1+\frac{4}{\alpha^{2}}\right) \sum_{i=1}^{n} x_{i} & 0 \\
\frac{1}{4}\left(1+\frac{4}{\alpha^{2}}\right) \sum_{i=1}^{n} x_{i} & \frac{1}{4}\left(1+\frac{4}{\alpha^{2}}\right) \sum_{i=1}^{n} x_{i}^{2} & 0 \\
0 & 0 & \frac{2 n}{\alpha^{2}}
\end{array}\right)
$$

Utilizando a distribuição normal assintótica dos estimadores de máxima verossimilhança $\hat{a}, \hat{b}$ e $\hat{\alpha}$, a qual é dada por (4.12), com $I(a, b, \alpha)$ dada por (4.33), é possivel calcular em forma fácil as variancias assintóticas (estimadas) dos estimadores de máxima verossimilhança $\widehat{a}, \hat{b}$ e $\hat{\alpha}$. Basta observar que o inverso da matriz de informação de Fisher é a matriz de variancia e covariancia de $\widehat{a}, \hat{b}$ e $\widehat{\alpha}$.(ver por exemplo, Kalfleisch, 1985).

Portanto, os intervalos de confiança para os parâmetros a, b e $\alpha$, considerando um nivel de confiança $100(1-\phi) \%$, são fornecidos por:

$$
\begin{aligned}
I C(a) & =\left[\widehat{a} \pm Z_{\phi / 2} \sqrt{v \hat{a} r(\widehat{a})}\right] \\
I C(b) & =\left[\widehat{b} \pm Z_{\phi / 2} \sqrt{v \hat{a} r(\widehat{b})}\right] \\
I C(\alpha) & =\left[\widehat{\alpha} \pm Z_{\phi / 2} \sqrt{v \widehat{a} r(\widehat{\alpha})}\right]
\end{aligned}
$$

onde $Z_{\phi / 2}$ é um percentil da distribuição Normal padronizada (ver por exemplo, Kalfleisch, 1985). 


\section{Capítulo 5}

\section{Análise Bayesiana do Modelo Log-Linear para a distribuição de Birnbaum-Saunders (considerando priori não-informativas).}

No capítulo 5 realizaremos uma análise Bayesiana do modeo Log-Linear da distribuição de Birnbaum-Saunders, considerando uma variável Stress $x_{i}$. Para representar - grau de conhecimento sobre os parâmetros utilizaremos densidades a priori não-informativas.

\subsection{Análise Bayesiana assumindo $\mathrm{a}, \mathrm{b}$ e $\alpha$ desco- nhecidos.}

Considerando o modelo Log-Linear (4.4) com uma variável stress $x_{i}$, utilizaremos duas densidades a priori não-informativas para representar o grau de conhecimento sobre os parâmetros $\mathrm{a}, \mathrm{b}$ e $\alpha$. A densidade a priori não-informativa de Jeffreys (ver por exemplo, Box e Tiao, 1973), dada por,

$$
P(a, b, \alpha) \propto|\operatorname{det} I(a, b, \alpha)|^{1 / 2},
$$

onde $I(a, b, \alpha)$ é a matriz de informação de Fisher para $\mathrm{a}, \mathrm{b}$ e $\alpha$. Para reduzir os calculos consideramos a matriz de informação de Fisher (4.33) em sua forma simplificada. Portanto 
a densidade a priori não informativa para a, b e $\alpha$, por (5.1) é, dada por,

$$
P(a, b, \alpha) \propto \frac{\left(4+\alpha^{2}\right)}{\alpha^{3}}
$$

onde $\alpha>0$ e $-\infty<a, b<\infty$.

Por outro lado por (4.25) e (4.26) observamos que $\alpha$ é ortogonal a a e b. Então outra densidade a priori não-informativa para a, b e $\alpha$ (ver Tibshirani, 1989) é dada por,

$$
P(a, b, \alpha) \propto\left(I_{\alpha, \alpha}(a, b, \alpha)\right)^{1 / 2} \times F(a, b),
$$

onde $I_{\alpha, \alpha}(a, b, \alpha)$ é o elemento da matriz de informação (4.33) do parâmetro de interesse $\left.\alpha\left(I_{\alpha, \alpha}(a, b, \alpha)\right)^{1 / 2} \propto \frac{1}{\alpha}\right)$, e $F(a, b)$ é uma função arbitraria positiva dos parâmetros perturbadores $a$ e $b(F(a, b)=1)$. Portanto uma densidade a priori não-informativa para $a$, $b$ e $\alpha$ por (5.3) é, dada por,

$$
P(a, b, \alpha) \propto \frac{1}{\alpha}
$$

onde $\alpha>0$ e $-\infty<a, b<\infty$, que também coincide com a priori não-informativa de Box e Tiao (1973) assumindo independência entre os parâmetros.

\subsubsection{Densidade a posteriori conjunta para a, b e $\alpha$.}

Combinando a função de verossimilhança (4.6) com as densidades a priori (5.2) e (5.4), obtemos a densidade a posteriori conjunta para a, b e $\alpha$ (ver por exemplo, Zellner, 1971) dada por,

$$
P(a, b, \alpha / D a d o s) \propto D\left(\alpha^{2}\right)\left(\prod_{i=1}^{n} W_{i}\right) \exp \left\{-\frac{1}{2} \sum_{i=1}^{n} Z_{i}^{2}\right\},
$$

para $\alpha>0$ e $-\infty<a, b<\infty$, onde $W_{i}$ e $Z_{i}$ são dados por (4.7), com

$$
D\left(\alpha^{2}\right)=\frac{\left(4+\alpha^{2}\right)}{\alpha^{n+3}} .
$$

se a densidade a priori for de Jeffreys, e $D\left(\alpha^{2}\right)=\frac{1}{\alpha^{n+1}}$, se a priori for dada em (5.4). 


\subsubsection{Densidade a posteriori marginal para $\alpha$.}

Em inferência Bayesiana, inferências são tipicamente baseadas nas densidades a posteriori marginais dos parâmetros considerados, em nosso casó $\mathrm{a}, \mathrm{b}$ e $\alpha$. As densidades a posteriori marginais para $\alpha$, a e b são obtidas por integração da densidade a posteriori conjunta (5.5) em $\alpha$, a ou b, respectivamente. Como estas integrais, em geral, não apresentam soluções analíticas explícitas, utilizamos o método de LAPLACE para aproximação de integrais (ver por exemplo, Kass, Tierney e Kadane, 1990).

A densidade a posteriori marginal para $\alpha$ considerando a densidade a priori não-informativa (5.2), pode ser escrita da seguinte forma:

$$
P(\alpha / \text { Dados }) \propto D\left(\alpha^{2}\right) \int_{-\infty}^{\infty} \int_{-\infty}^{\infty} f(a, b) \exp [-n h(a, b)] d a d b
$$

onde,

$$
f(a, b)=\prod_{i=1}^{n} \cosh \left[\frac{y_{i}-a-b x_{i}}{2}\right]
$$

e

$$
n h(a, b)=\frac{1}{2} \sum_{i=1}^{n} Z_{i}^{2}
$$

com $Z_{i}$ dado por (4.7).

Portanto, utilizando a aproximação de LAPLACE em (5.6) (ver, apêndice A) , temos que a densidade a posteriori marginal aproximada para $\alpha$, é dada por,

$$
P(\alpha / D a d o s) \propto\left(4+\alpha^{2}\right) \alpha^{-(n+1)} \exp \left\{-\frac{2 A(\widehat{a}, \widehat{b})}{\alpha^{2}}\right\}
$$

para $\alpha>0$, com

$$
A(\widehat{a}, \hat{b})=\sum_{i=1}^{n} \operatorname{senh}^{2}\left[\frac{y_{i}-\hat{a}-\hat{b} x_{i}}{2}\right]
$$

onde $\widehat{a}$ e $\widehat{b}$ são soluções das seguintes equações:

$$
\left\{\begin{array}{l}
\sum_{i=1}^{n} \operatorname{senh}\left(y_{i}-\hat{a}-\hat{b} x_{i}\right)=0 \\
\sum_{i=1}^{n} x_{i} \operatorname{senh}\left(y_{i}-\hat{a}-\hat{b} x_{i}\right)=0
\end{array}\right.
$$


Ou seja para determinar $\hat{a}$ e $\hat{b}$ que maximizem $\mathrm{nh}(\mathrm{a}, \mathrm{b})$ teremos que resolver (5.8) numéricamente.

Similarmente, considerando a densidade a priori não-informativa (5.4), e utilizando aproximação de LAPLACE e o resultado (3.1), a densidade a posteriori marginal aproximada para $\alpha$ é dada por,

$$
P(\alpha / D a d o s)=\frac{2^{n / 2} A^{\frac{n-2}{2}}(\widehat{a}, \widehat{b})}{\Gamma\left(\frac{n-2}{2}\right)} \alpha^{-(n-1)} \exp \left\{-\frac{2 A(\hat{a}, \hat{b})}{\alpha^{2}}\right\},
$$

onde $\alpha>0$; e $\hat{a}$ e $\widehat{b}$ são dados em (5.8).

A moda da densidade a posteriori marginal (5.9) é dada por

$$
\widehat{\alpha}^{*^{2}}=\frac{4 A(\widehat{a}, \widehat{b})}{n-1}
$$

onde $A(\hat{a}, \hat{b})$ é dado em (5.7). Para calcular a moda basta derivar o logaritmo de (5.9) em relação a $\alpha$, igualar a zero e resolver em $\alpha$.

\subsubsection{Densidade a posteriori marginal conjunta para a e b.}

A densidade a posteriori marginal conjunta para a e b considerando a priori não-informativa (5.2), pode ser expressa da seguinte forma,

$$
P(a, b / D a d o s) \propto \prod_{i=1}^{n} \cosh \left[\frac{y_{i}-a-b x_{i}}{2}\right] \int_{0}^{\infty} f(\alpha) \exp [-n h(\alpha)] d \alpha
$$

onde,

$$
f(\alpha)=\frac{4+\alpha^{2}}{\alpha^{3}}
$$

e

$$
n h(\alpha)=n \ln \alpha+\frac{2}{\alpha^{2}} \sum_{i=1}^{n} \operatorname{senh}^{2}\left[\frac{y_{i}-a-b x_{i}}{2}\right]
$$

Portanto, utilizando a aproximação de LAPLACE, temos:

$$
P(a, b / D a d o s) \propto\left\{\prod_{i=1}^{n} \cosh \left[\frac{y_{i}-a-b x_{i}}{2}\right]\right\}\left\{\frac{1+\frac{1}{n} \sum_{i=1}^{n} \operatorname{senh}^{2}\left[\frac{y_{i}-a-b x_{i}}{2}\right]}{\left(\sum_{i=1}^{n} \operatorname{senh}^{2}\left[\frac{y_{i}-a-b x_{i}}{2}\right]\right)^{\frac{(n+2)}{2}}}\right\},
$$


para $-\infty<a, b<\infty$

Por outro lado, a densidade a posteriori marginal conjunta para a e b considerando a densidade a priori não-informativa (5.4), pode ser escrita da seguinte forma,

$$
P(a, b / D a d o s) \propto \prod_{i=1}^{n} \cosh \left[\frac{y_{i}-a-b x_{i}}{2}\right] \int_{0}^{\infty} f(\alpha) \exp [-n h(\alpha)] d \alpha,
$$

onde,

$$
f(\alpha)=\frac{1}{\alpha}
$$

e

$$
n h(\alpha)=n \ln \alpha+\frac{2}{\alpha^{2}} \sum_{i=1}^{n} \operatorname{senh}^{2}\left[\frac{y_{i}-a-b x_{i}}{2}\right]
$$

Assim utilizando a aproximação de LAPLACE, temos que:

$$
P(a, b / D a d o s) \propto \frac{\prod_{i=1}^{n} \cosh \left[\frac{y_{i}-a-b x_{i}}{2}\right]}{\left(\sum_{i=1}^{n} \operatorname{senh}^{2}\left[\frac{y_{i}-a-b x_{i}}{2}\right]\right)^{\frac{n}{2}}}
$$

para $-\infty<a, b<\infty$

\subsection{Uma análise Bayesiana assumindo $\alpha$ conhecido.} a e b, dada por,

Assumindo $\alpha$ conhecido, de (4.6), obtemos a função de verossimilhança para

$$
L(a, b) \propto\left\{\prod_{i=1}^{n} \cosh \left[\frac{y_{i}-a-b x_{i}}{2}\right]\right\} \exp \left\{-\frac{2}{\alpha^{2}} \sum_{i=1}^{n} \operatorname{senh}^{2}\left[\frac{y_{i}-a-b x_{i}}{2}\right]\right\} .
$$

Considerar uma densidade a priori não-informativa para a e b, localmente uniforme dada por,

$$
P(a, b) \propto \text { constante, }
$$

para $-\infty<a, b<\infty$ 
Assim por (5.15) e.(5.16) a densidade a posteriori conjunta para a e b é,

$$
P(a, b / D a d o s) \propto\left\{\prod_{i=1}^{n} \cosh \left[\frac{y_{i}-a-b x_{i}}{2}\right]\right\} \exp \left\{-\frac{1}{2} \sum_{i=1}^{n} Z_{i}^{2}\right\}
$$

onde $-\infty<a, b<\infty$.

\subsubsection{Densidade a posteriori marginal para a e b.}

A densidade a posteriori marginal para a, considerando a densidade a priori não-informativa (5.16), pode ser escrita da seguinte forma:

$$
P(a / D a d o s) \propto \int_{-\infty}^{\infty} f(b) \exp [-n h(b)] d b,
$$

onde,

$$
f(b)=\prod_{i=1}^{n} \cosh \left[\frac{y_{i}-a-b x_{i}}{2}\right]
$$

e.

$$
n h(b)=\frac{2}{\alpha^{2}} \sum_{i=1}^{n} \operatorname{senh}^{2}\left[\frac{y_{i}-a-b x_{i}}{2}\right] .
$$

Portanto, utilizando a aproximação de LAPLACE em (5.18), obtemos a densidade a posteriori marginal aproximada para a, dada por,

$$
P(a / D a d o s) \propto \frac{\left\{\prod_{i=1}^{n} \cosh \left[\frac{y_{i}-a-\widehat{b} x_{i}}{2}\right]\right\}}{\left(\sum_{i=1}^{n} x_{i}^{2} \cosh \left[y_{i}-a-\widehat{b} x_{i}\right]\right)^{1 / 2}} \exp \left\{-\frac{2}{\alpha^{2}} \sum_{i=1}^{n} B Z_{i}\right\},
$$

onde $-\infty<a<\infty, B Z_{i}=\operatorname{senh}^{2}\left[\frac{y_{i}-a-\widehat{b} x_{i}}{2}\right]$ e $\hat{b}$ é dado por,

$$
\sum_{i=1}^{n} x_{i} e^{y_{i}-a} e^{-\widehat{-b} x_{i}}=\sum_{i=1}^{n} x_{i} e^{-\left(y_{i}-a\right)} e^{\widehat{b} x_{i}} .
$$

Similarmente a densidade a posteriori marginal para $b$, pode ser expressa na seguinte forma:

$$
P(b / D a d o s) \propto \int_{-\infty}^{\infty} f(a) \exp [-n h(a)] d a,
$$


onde,

$$
f(a)=\prod_{i=1}^{n} \cosh \left[\frac{y_{i}-a-b x_{i}}{2}\right]
$$

e

$$
n h(a)=\frac{2}{\alpha^{2}} \sum_{i=1}^{n} \operatorname{senh}^{2}\left[\frac{y_{i}-a-b x_{i}}{2}\right]
$$

Portanto, utilizando a aproximação de LAPLACE em (5.20), obtemos a densidade a posteriori marginal aproximada para $b$, dada por,

$$
P(b / D a d o s) \propto \frac{\left\{\prod_{i=1}^{n} \cosh \left[\frac{y_{i}-\hat{a}-b x_{i}}{2}\right]\right\}}{\left(\sum_{i=1}^{n} \cosh \left[y_{i}-\widehat{a}-b x_{i}\right]\right)^{1 / 2}} \exp \left\{-\frac{2}{\alpha^{2}} \sum_{i=1}^{n} \operatorname{senh}^{2}\left[\frac{y_{i}-\widehat{a}-b x_{i}}{2}\right]\right\},
$$

onde $-\infty<b<\infty$, e $\hat{a}$ é dado por,

$$
\widehat{a}=\frac{1}{2} \ln \left\{\frac{\sum_{i=1}^{n} e^{y_{i}-b x_{i}}}{\sum_{i=1}^{n} e^{-\left(y_{i}-b x_{i}\right)}}\right\},
$$

para cada valor de b.

\subsubsection{Densidade a posteriori para o tempo medio de vida num nivel de Stress especificado $X^{*}$.}

As industrias de materiais que trabalham com fadigas, usualmente tem interesse em inferir sobre o tempo médio de sobrevivência ou tempo médio de vida da fadiga $\lambda=E\left[Y^{*}\right]=a+b x^{*}$, considerando um nivel de Stress $x^{*}$.

dada por

Portanto, assumindo $\alpha$ conhecido, consideraramos a transformação de variáveis

$$
\lambda=a+b x^{*}
$$

$\mathrm{e} b=b$. Assim considerando a priori localmente uniforme para a e b (5.16), a densidade a posteriori conjunta para $\lambda$ e $b$ é dada por, 


$$
\begin{aligned}
P(\lambda, b / D a d o s) \propto & \left\{\prod_{i=1}^{n} \cosh \left[\frac{y_{i}-\lambda+b\left(x^{*}-x_{i}\right)}{2}\right]\right\} \times \\
& \times \exp \left\{-\frac{2}{\alpha^{2}} \sum_{i=1}^{n} \operatorname{senh}^{2}\left[\frac{y_{i}-\lambda+b\left(x^{*}-x_{i}\right)}{2}\right]\right\},
\end{aligned}
$$

onde $-\infty<\lambda<\infty$.

Por conseguinte a densidade a posteriori marginal para $\lambda$, considerando a densidade a priori não-informativa (5.16), pode ser escrita da seguinte forma:

$$
P(\lambda / D a d o s) \propto \int_{-\infty}^{\infty} f(b) \exp [-n h(b)] d b
$$

onde,

$$
f(b)=\prod_{i=1}^{n} \cosh \left[\frac{y_{i}-\lambda+b\left(x^{*}-x_{i}\right)}{2}\right]
$$

e

$$
n h(b)=\frac{2}{\alpha^{2}} \sum_{i=1}^{n} \operatorname{senh}^{2}\left[\frac{y_{i}-\lambda+b\left(x^{*}-x_{i}\right)}{2}\right]
$$

Portanto, utilizando a aproximação de LAPLACE em (5.23), obtemos a densidade a posteriori marginal aproximada para $\lambda$, dada por,

$$
\begin{aligned}
P(\lambda / \text { Dados }) \propto & \frac{\left\{\prod_{i=1}^{n} \cosh \left[\frac{y_{i}-\lambda+\hat{b}\left(x^{*}-x_{i}\right)}{2}\right]\right\}}{\left(\sum_{i=1}^{n}\left(x^{*}-x_{i}\right)^{2} \cosh \left[y_{i}-\lambda+\hat{b}\left(x^{*}-x_{i}\right)\right]\right)^{1 / 2}} \times \\
& \times \exp \left\{-\frac{2}{\alpha^{2}} \sum_{i=1}^{n} \operatorname{senh}^{2}\left[\frac{y_{i}-\lambda+\hat{b}\left(x^{*}-x_{i}\right)}{2}\right]\right\}
\end{aligned}
$$

onde $-\infty<\lambda<\infty$, e $\hat{b}$ é dado por,

$$
x^{*} \sum_{i=1}^{n} \operatorname{senh}\left[y_{i}-\lambda+\hat{b}\left(x^{*}-x_{i}\right)\right]=\sum_{i=1}^{n} x_{i} \operatorname{senh}\left[y_{i}-\lambda+\widehat{b}\left(x^{*}-x_{i}\right)\right] \text {. }
$$




\subsubsection{Densidade preditiva para uma observação futura}

Em muitas ocasiões, dada nossa informação amostral (Dados), podemos ter interesse em fazer inferências sobre uma observação que ainda não foi observada, isto é, fazer uma previsão para uma observação futura. Na análise Bayesiana a função de densidade de Probabilidade para uma observação ainda não-observada, dada nossa informação amostral, pode ser obtida e é conhecida como densidade preditiva para uma observação futura, representada por exemplo com $Y_{(n+1)}^{*}$. Assumindo $\alpha$ conhecido, a densidade preditiva para uma observação futura $Y_{(n+1)}^{*}$ (ver por exemplo, Press, 1989) é dada por,

$$
f\left(y_{(n+1)}^{*} / D a d o s\right)=\int_{-\infty}^{\infty} \int_{-\infty}^{\infty} f\left(y_{(n+1)}^{*} / a, b\right) P(a, b / D a d o s) d a d b
$$

onde $P(a, b /$ Dados $)$ é dada por (5.17), e

$$
f\left(y_{(n+1)}^{*} / a, b\right)=\frac{1}{2 \sqrt{2 \pi} \alpha} \cosh \left[H_{y^{*}}\right] \exp \left\{-\frac{2}{\alpha^{2}} \operatorname{senh}^{2}\left[H_{y^{*}}\right]\right\} .
$$

Observe que,

$$
f\left(y_{(n+1)}^{*} / D a d o s\right) \propto \int_{-\infty}^{\infty} \int_{-\infty}^{\infty} f_{x^{*}}(a, b) e^{-n h_{x^{*}}(a, b)} d a d b
$$

onde,

$$
f_{x^{*}}(a, b)=\cosh \left[H_{y^{*}}\right] \prod_{i=1}^{n} \cosh \left[H_{y}\right]
$$

e

$$
n h_{x^{*}}(a, b)=\frac{2}{\alpha^{2}}\left\{\operatorname{senh}^{2}\left[H_{y^{*}}\right]+\sum_{i=1}^{n} \operatorname{senh}^{2}\left[H_{y}\right]\right\}
$$

com

$$
H_{y^{*}}=\frac{y_{(n+1)}^{*}-a-b x^{*}}{2}
$$

e

$$
H_{y}=\frac{y_{i}-a-b x_{i}}{2} .
$$


Portanto, utilizando a aproximação de LAPLACE em (5.27) (ver, apêndice A), a densidade preditiva para uma observação futura $y_{(n+1)}^{*}$, é dada por,

$$
\begin{aligned}
f\left(y_{(n+1)}^{*} / \text { Dados }\right) \propto & \frac{\cosh \left[\frac{H_{\hat{y}^{*}}}{2}\right] \prod_{i=1}^{n} \cosh \left[\frac{H_{\hat{y}}}{2}\right]}{B\left(y_{(n+1)}^{*}\right)} \times \\
& \times \exp \left\{-\frac{2}{\alpha^{2}}\left(\operatorname{senh}^{2}\left[\frac{H_{\widehat{y}^{*}}}{2}\right]+\sum_{i=1}^{n} \operatorname{senh}^{2}\left[\frac{H_{\hat{y}}}{2}\right]\right)\right\},
\end{aligned}
$$

onde $y_{(n+1)}^{*}>0 \mathrm{e}$

$$
B\left(y_{(n+1)}^{*}\right)=\left[\left(\cosh \left[H_{\widehat{y}^{*}}\right]+v_{0}\right)\left(\left(x^{*}\right)^{2} \cosh \left[H_{\hat{y}^{*}}\right]+v_{2}\right)-\left(x^{*} \cosh \left[H_{\hat{y}^{*}}\right]+v_{1}\right)^{2}\right]^{1 / 2},
$$

e

$$
v_{l}=\sum_{i=1}^{n} x_{i}^{l} \cosh \left(H_{\widehat{y}}\right), l=0,1,2
$$

com

$$
H_{\widehat{y}^{*}}=y_{(n+1)}^{*}-\widehat{a}-\hat{b} x *
$$

e

$$
H_{\widehat{y}}=y_{i}-\widehat{a}-\widehat{b} x_{i}
$$

A densidade preditiva (5.28) pode ser utilizada para obter uma previsão pontual, por exemplo a média ou a moda, ou para construir um intervalo de credibilidade da previsão. 


\section{Capítulo 6}

\section{Análise Bayesiana do Modelo Log-Linear para a distribuição de Birnbaum-Saunders (considerando densidades a priori informativas).}

Neste capítulo apresentaremos uma análise Bayesiana do modelo Log-Linear da distribuição de Birnbaum-Saunders, considerando uma variável Stress $x_{i}$. Para representar o grau de conhecimento sobre os parâmetros utilizaremos densidades a priori informativas.

\subsection{Análise Bayesiana assumindo a, b e $\alpha$ desco- nhecidos.}

Usualmente os projetos de engenharia de materiais são processos evolucionários, devido à grandes modificações e inovações necessarias para se adequar as novas exigências na área industrial. Assim a informação de projetos anteriores pode ser utilizada como uma tentativa para melhorar os resultados de um novo projeto, isto é, utilizando informação a priori.

Utilizando o modelo Log-Linear da distribuição de Birnbaum-Saunders com uma variavel Stress $x_{i}$, consideraremos densidades a priori informativas para representar 
o grau de conhecimento sobre os parâmetros $a, b$ e $\alpha$. Como a priori conjunta para $a, b$ e $\alpha$ pode ser escrita por,

$$
P\left(a, b, \alpha / a_{1}, b_{1}, \sigma_{1}, \sigma_{2}, \rho, c_{1}, d_{1}\right)=P\left(a, b / \alpha, a_{1}, b_{1}, \sigma_{1}, \sigma_{2}, \rho\right) P\left(\alpha / c_{1}, d_{1}\right),
$$

para $\alpha>0$ e $-\infty<a, b<\infty$, consideramos uma priori informativa para $a, b$ e $\alpha$ dada pelo produto de uma distribuição normal bivariada com médias e variancias conhecidas com uma distribuição gama com parâmetros conhecidos. Estas densidades a priori são dadas por,

$$
P\left(a, b / a_{1}, b_{1}, \sigma_{1}, \sigma_{2}, \rho\right)=P N
$$

onde

$$
P N=\frac{1}{2 \pi \sigma_{1} \sigma_{2} \sqrt{1-\rho^{2}}} \exp \{N O R\}
$$

com

$$
\begin{gathered}
\text { NOR }=\frac{-1}{2\left(1-\rho^{2}\right)}\left[(N O 1)^{2}-2 \rho(N O 1)(N O 2)+(N O 2)^{2}\right] \\
N O 1=\frac{a-a_{1}}{\sigma 1}
\end{gathered}
$$

e

$$
N O 2 \doteq \frac{b-b_{1}}{\sigma 2}
$$

para $-\infty<a, b<\infty$, $\mathrm{e}$

onde

$$
P\left(\alpha / c_{1}, d_{1}\right)=P G
$$

$$
P G=\frac{d_{1}^{c_{1}}}{\Gamma\left(c_{1}\right)} \alpha^{\left(c_{1}-1\right)} \exp \left\{-d_{1} \alpha\right\}
$$

para $\alpha>0$, onde (6.2) é uma distribuição normal bivariada com $a_{1}, b_{1}, \sigma_{1}, \sigma_{2}$ e $\rho$ conhecidos e (6.3) é uma gama com $c_{1}$ e $d_{1}$ conhecidos.

A escolha subjetiva dessas densidadès a priori foi realizada apartir de algumas considerações empíricas dos métodos estatísticos para resistência, tempo de vida e limite de fadiga dos materiais (ver por exemplo, Souza 1982; e ver Birnbaum e Saunders, 1958). 


\subsubsection{Densidade a posteriori conjunta para $a, b$ e $\alpha$.}

Do produto da função de verossimilhançà (4.6) com a densidade a priori informativa (6.1) obtemos a densidade a posteriori conjunta para a, b e $\alpha$ (ver por exemplo, Zellner, 1971) dada por,

$$
P(a, b, \alpha / P C) \propto \frac{(P N)(P G)}{\alpha^{n}}\left(\prod_{i=1}^{n} A W_{i}\right) \exp \left\{-\frac{1}{2} \sum_{i=1}^{n} Z_{i}^{2}\right\},
$$

onde $Z_{i}$ é dado em (4.7), com $P C=\left(\right.$ Dados, $\left.a_{1}, b_{1}, \sigma_{1}, \sigma_{2}, c_{1}, d_{1}, \rho\right) \mathrm{e}$

$$
A W_{i}=\cosh \left[\frac{y_{i}-a-b x_{i}}{2}\right]
$$

para $\alpha>0$ e $-\infty<a, b<\infty$.

\subsubsection{Densidades a posteriori marginais para $\alpha, a$ e $b$.}

As densidades a posteriori marginais para $\alpha$, a e b são obtidas por integração da densidade a posteriori conjunta (6.4) em $\alpha$, a ou b, respectivamente. Assim a densidade a posteriori marginal para $\alpha$ considerando a densidade a priori informativa (6.1), pode ser escrita da seguinte forma:

$$
P(\alpha / P C) \propto \frac{P G}{\alpha^{n}} \int_{-\infty}^{\infty} \int_{-\infty}^{\infty} f(a, b) \exp [-n h(a, b)] d a d b
$$

onde,

$$
f(a, b)=(P N) \prod_{i=1}^{n} \cosh \left[\frac{y_{i}-a-b x_{i}}{2 \cdot}\right]
$$

e

$$
n h(a, b)=\frac{1}{2} \sum_{i=1}^{n} Z_{i}^{2}
$$

Utilizando a aproximação de LAPLACE em (6.5), encontramos a densidade a posteriori marginal aproximada para $\alpha$, dada por,

$$
P(\alpha / P C) \propto \alpha^{-\left(\left(n-c_{1}\right)-1\right)} \exp \left\{-\frac{2 A(\hat{a}, \hat{b})}{\alpha^{2}}-d_{1} \alpha\right\}
$$


para $\alpha>0$, com

$$
A(\widehat{a}, \hat{b})=\sum_{i=1}^{n} \operatorname{senh}^{2}\left[\frac{y_{i}-\widehat{a}-\hat{b} x_{i}}{2}\right]
$$

onde $\widehat{a}$ e $\widehat{b}$ são soluções das seguintes equações:

$$
\left\{\begin{array}{l}
\sum_{i=1}^{n} \operatorname{senh}\left(y_{i}-\widehat{a}-\hat{b} x_{i}\right)=0 \\
\sum_{i=1}^{n} x_{i} \operatorname{senh}\left(y_{i}-\widehat{a}-\hat{b} x_{i}\right)=0 .
\end{array}\right.
$$

Assim, para determinar $\widehat{a}$ e $\hat{b}$ que maximizem $\operatorname{nh}(\mathrm{a}, \mathrm{b})$ teremos que resolver (6.7), usando um método numérico.

A densidade a posteriori marginal conjunta para a e b considerando a priori informativa (6.1), pode ser expressa da seguinte forma,

$$
P(a, b / P C) \propto P N \prod_{i=1}^{n} \cosh \left[\frac{y_{i}-a-b x_{i}}{2}\right] \int_{0}^{\infty} f(\alpha) \exp [-n h(\alpha)] d \alpha
$$

onde,

$$
f(\alpha)=\left(c_{1}+1\right) \ln \alpha-d_{1} \alpha
$$

$\mathbf{e}$

$$
n h(\alpha)=n \ln \alpha+\frac{2}{\alpha^{2}} \sum_{i=1}^{n} \operatorname{senh}^{2}\left[\frac{y_{i}-a-b x_{i}}{2}\right]
$$

Portanto, utilizando a aproximação de LAPLACE, temos:

$$
P(a, b / P C) \propto\left\{\frac{P N \prod_{i=1}^{n} \cosh \left[\frac{y_{i}-a-b x_{i}}{2}\right]}{\left(\sum_{i=1}^{n} \operatorname{senh}^{2}\left[\frac{y_{i}-a-b x_{i}}{2}\right]\right)^{\frac{\left(n-c_{1}-2\right)}{2}}}\right\} \exp \left\{-d_{1}\left[\frac{4}{n} \sum_{i=1}^{n} D Z_{i}\right]^{\frac{1}{2}}\right\},
$$

para $-\infty<a, b<\infty, \operatorname{com} D Z_{i}=\operatorname{senh}^{2}\left[\frac{y_{i}-a-\widehat{\delta}_{i}}{2}\right]$. 


\subsection{Uma análise Bayesiana assumindo $\alpha$ conhecido.} a e b, dada por,

Assumindo $\alpha$ conhecido, de (4.6), obtemos a função de verossimilhança para

$$
L(a, b) \propto\left\{\prod_{i=1}^{n} \cosh \left[\frac{y_{i}-a-b x_{i}}{2}\right]\right\} \exp \left\{-\frac{2}{\alpha^{2}} \sum_{i=1}^{n} \operatorname{senh}^{2}\left[\frac{y_{i}-a-b x_{i}}{2}\right]\right\} .
$$

De (6.10) e (6.2), a densidade a posteriori conjunta para a e b, é dada por,

$$
P(a, b / P C 1) \propto\left\{\prod_{i=1}^{n} \cosh \left[\frac{y_{i}-a-b x_{i}}{2}\right]\right\} \exp \left\{-\frac{1}{2} \sum_{i=1}^{n} Z_{i}^{2}\right\} \times P N
$$

para $-\infty<a, b<\infty$, onde $P C 1=\left(\right.$ Dados $\left., a_{1}, b_{1}, \sigma_{1}, \sigma_{2}, \rho\right)$.

\subsubsection{Densidade a posteriori marginal para $a$ e $b$.}

A densidade a posteriori marginal para a, considerando a densidade a priori informativa (6.2), pode ser escrita da seguinte forma:

$$
P(a / P C 1) \propto \int_{-\infty}^{\infty} f(b) \exp [-n h(b)] d b
$$

onde,

$$
f(b)=P N \prod_{i=1}^{n} \cosh \left[\frac{y_{i}-a-b x_{i}}{2}\right]
$$

e

$$
n h(b)=\frac{2}{\alpha^{2}} \sum_{i=1}^{n} \operatorname{senh}^{2}\left[\frac{y_{i}-a-b x_{i}}{2}\right] .
$$

Portanto, utilizando a aproximação de LAPLACE em (6.12), obtemos a densidade a posteriori marginal aproximada para a, dada por,

$$
P(a / P C 1) \propto \widehat{B P N} \frac{\left\{\prod_{i=1}^{n} \cosh \left[\frac{y_{i}-a-\widehat{b} x_{i}}{2}\right]\right\}}{\left(\sum_{i=1}^{n} x_{i}^{2} \cosh \left[y_{i}-a-\widehat{b} x_{i}\right]\right)^{1 / 2}} \exp \left\{-\frac{2}{\alpha^{2}} \sum_{i=1}^{n} B Z_{i}\right\}
$$


onde $-\infty<a<\infty, B Z_{i}=\operatorname{senh}^{2}\left[\frac{y_{i}-a-\widehat{b} x_{i}}{2}\right]$ e $\hat{b} \dot{\mathrm{e}}$ dado por

$$
\sum_{i=1}^{n} x_{i} e^{y_{i}-a} e^{-\widehat{b} x_{i}}=\sum_{i=1}^{n} x_{i} e^{-\left(y_{i}-a\right)} e^{\hat{b} x_{i}}
$$

com

$$
\widehat{B P N}=\frac{1}{2 \pi \sigma_{1} \sigma_{2} \sqrt{1-\rho^{2}}} \exp \{N \widehat{O R} B\}
$$

onde

$$
\begin{gathered}
N \widehat{O R} B=\frac{-1}{2\left(1-\rho^{2}\right)}\left[(N O 1)^{2}-2 \rho(N O 1)(N \widehat{O 2} B)+(N \widehat{O 2} B)^{2}\right] \\
N O 1=\frac{a-a_{1}}{\sigma 1}
\end{gathered}
$$

e

$$
\widehat{N O 2} B=\frac{\widehat{b}-b_{1}}{\sigma 2}
$$

Similarmente a densidade a posteriori marginal para $b$, pode ser expressa na seguinte forma:

$$
P(b / P C 1) \propto \int_{-\infty}^{\infty} f(a) \exp [-n h(a)] d a,
$$

onde,

$$
f(a)=P N \prod_{i=1}^{n} \cosh \left[\frac{y_{i}-a-b x_{i}}{2}\right]
$$

e

$$
n h(a)=\frac{2}{\alpha^{2}} \sum_{i=1}^{n} \operatorname{senh}^{2}\left[\frac{y_{i}-a-b x_{i}}{2}\right]
$$


Portanto, utilizando a aproximação de LAPLACE em (6.14), obtemos a densidade a posteriori marginal aproximada para $b$, dada por,

$$
P(b / P C 1) \propto \widehat{A P N} \frac{\left\{\prod_{i=1}^{n} \cosh \left[\frac{y_{i}-\widehat{a}-b x_{i}}{2}\right]\right\}}{\left(\sum_{i=1}^{n} \cosh \left[y_{i}-\widehat{a}-b x_{i}\right]\right)^{1 / 2}} \exp \left\{-\frac{2}{\alpha^{2}} \sum_{i=1}^{n} A Z_{i}\right\}
$$

onde $-\infty<b<\infty, A Z_{i}=\operatorname{senh}^{2}\left[\frac{y_{i}-\hat{a}-b x_{i}}{2}\right]$ e $\widehat{a}$ é dado por,

$$
\widehat{a}=\frac{1}{2} \ln \left\{\frac{\sum_{i=1}^{n} e^{y_{i}-b x_{i}}}{\sum_{i=1}^{n} e^{-\left(y_{i}-b x_{i}\right)}}\right\},
$$

para cada valor de b. Com

$$
\widehat{A P N}=\frac{1}{2 \pi \sigma_{1} \sigma_{2} \sqrt{1-\rho^{2}}} \exp \{N \widehat{O R} A\}
$$

onde

$$
\begin{gathered}
N \widehat{O R} A=\frac{-1}{2\left(1-\rho^{2}\right)}\left[(N \widehat{O 1} A)^{2}-2 \rho(N \widehat{O 1} A)(N O 2)+(N O 2)^{2}\right], \\
N \widehat{O 1} A=\frac{\widehat{a}-a_{1}}{\sigma 1},
\end{gathered}
$$

e

$$
N O 2=\frac{b-b_{1}}{\sigma 2}
$$

\subsubsection{Densidade preditiva para uma observação futura}

Dada a informação amostral, podemos fazer uma previsão para uma observação não-observada. Na análise Bayesiana a função de densidade de probabilidade para uma observação ainda não-observada, dada nossa informação amostral é conhecida como densidade preditiva para uma observação futura. Assumindo $\alpha$ conhecido, a densidade preditiva para uma observação futura $Y_{(n+1)}^{* *}$ (ver por exèmplo, Press, 1989) é dada por,

$$
f\left(y_{(n+1)}^{* *} / D a d o s\right)=\int_{-\infty}^{\infty} \int_{-\infty}^{\infty} f\left(y_{(n+1)}^{* *} / a, b\right) P(a, b / P C 1) d a d b
$$


onde $P(a, b / P C 1)$ é dada por (6.11), e

$$
f\left(y_{(n+1)}^{* *} / a, b\right)=\frac{1}{2 \sqrt{2 \pi} \alpha} \cosh \left[H_{y^{* *}}\right] \exp \left\{-\frac{2}{\alpha^{2}} \operatorname{senh}^{2}\left[H_{y^{* *}}\right]\right\} .
$$

Observe que,

$$
f\left(y_{(n+1)}^{* *} / D a d o s\right) \propto \int_{-\infty}^{\infty} \int_{-\infty}^{\infty} f_{x^{* *}}(a, b) e^{-n h_{x^{* *}(a, b)}} d a d b
$$

onde,

$$
f_{x^{* *}}(a, b)=\cosh \left[H_{y^{* *}}\right] \prod_{i=1}^{n} \cosh \left[H_{y}\right] \times P N
$$

e

$$
n h_{x * *}(a, b)=\frac{2}{\alpha^{2}}\left\{\operatorname{senh}^{2}\left[H_{y^{*}}\right]+\sum_{i=1}^{n} \operatorname{senh}^{2}\left[H_{y}\right]\right\}
$$

com

$$
H_{y^{* *}}=\frac{y_{(n+1)}^{* *}-a-b x^{*}}{2}
$$

e

$$
H_{y}=\frac{y_{i}-a-b x_{i}}{2}
$$

Portanto, utilizando a aproximação de LAPLACE em (6.18) (ver, apêndice A), a densidade preditiva para uma observação futura $Y_{(n+1)}^{* *}$, é dada por,

$$
\begin{aligned}
f\left(y_{(n+1)}^{* *} / D a d o s\right) \propto & \frac{\cosh \left[\frac{H_{\hat{y}^{* *}}}{2}\right] \prod_{i=1}^{n} \cosh \left[\frac{H_{\widehat{y}}}{2}\right]}{B\left(y_{(n+1)}^{* *}\right)}(\widehat{P N}) \times \\
& \times \exp \left\{-\frac{2}{\alpha^{2}}\left(\operatorname{senh}^{2}\left[\frac{H_{\widehat{y}} \cdot}{2}\right]+\sum_{i=1}^{n} \operatorname{senh}^{2}\left[\frac{H_{\widehat{y}}}{2}\right]\right)\right\},
\end{aligned}
$$




$$
\begin{aligned}
& \text { para } y_{(n+1)}^{* *}>0 \\
& B\left(y_{(n+1)}^{* *}\right)=\left[\left(\cosh \left[H_{\widehat{y}^{* *}}\right]+v_{0}\right)\left(\left(x^{*}\right)^{2} \cosh \left[H_{\widehat{y}^{* *}}\right]+v_{2}\right)-\left(x^{*} \cosh \left[H_{\widehat{y}^{*}}\right]+v_{1}\right)^{2}\right]^{1 / 2}
\end{aligned}
$$

e

$$
v_{l}=\sum_{i=1}^{n} x_{i}^{l} \cosh \left(H_{\hat{y}}\right), l=0,1,2
$$

com

$$
H_{\hat{y}^{* *}}=y_{(n+1)}^{*}-\widehat{a}-\hat{b} x *
$$

e

$$
H_{\widehat{y}}=y_{i}-\widehat{a}-\widehat{b} x_{i} .
$$

Onde

$$
\widehat{P N}=\frac{1}{2 \pi \sigma_{1} \sigma_{2} \sqrt{1-\rho^{2}}} \exp \{\widehat{N O} R\}
$$

com

$$
\begin{aligned}
& \widehat{N O R}=\frac{-1}{2\left(1-\rho^{2}\right)}\left[(\widehat{N O} 1)^{2}-2 \rho(\widehat{N O} 1)(\widehat{N O} 2)+(\widehat{N O} 2)^{2}\right] \\
& \widehat{N O 1}=\frac{\widehat{a}-a_{1}}{\sigma 1},
\end{aligned}
$$

e

$$
\widehat{N O 2}=\frac{\widehat{b}-b_{1}}{\sigma 2}
$$

A expressão (6.19) pode ser utilizada para obter estimativas pontuais ou estimativas por intervalos da previsão. 


\section{Capitulo 7}

\section{Análise da Variancia (ANOVA).}

No capítulo 7, realizaremos um estudo sobre planejamentos de experimentos de dois fatores (classificação de dois critérios), sob um modelo Log-Linear para a distribuição de Birnbaum-Saunders. Na prática geralmente nos referimos à duas classificações ou fatores em um experimento como tratamentos e blocos, mas nada impede, obviamente, que designemos por fator 1 e fator2, etc. (ver por exemplo, Guttman, 1982).

O objetivo de muitos experimentos é determinar o efeito de mais de um fator em uma variável resposta Y. Por exemplo, podemos estar interessados em determinar o efeito das variávejs pressão e temperatura, no tempo de vida de um material.

O motivo deste estudo é dado pela falta de adequabilidade da distribuição normal em análise de experimentos na área de confiabilidade e testes de sobrevivencia industriais. A distribuição de Birnbaum-Saunders pode. ser de grande utilidade nessas aplicações (ver por exemplo, Fries e Bhattacharyya, 1983).

\subsection{Caso geral do modelo.}

\subsubsection{Estimadores de máxima verossimilhança.}

Supondo que temos uma variável tratamento $\mathrm{A}$ com I niveis e uma variável bloco B com J níveis, onde se admite que haja um valor experimental correspondente a cada tratamento e bloco, para o $\mathrm{j}$-ésimo tratamento e o $\mathrm{j}$-ésimo bloco, denotamos este 
valor por $y_{i j k}$, para $i=1, \ldots, l$, para $j=1, \ldots, j$, para $k=1, \ldots, n$, onde $\mathrm{k}$ é o número de repetições ou réplicas em cada $\mathrm{i}$-ésimo tratamento e $\mathrm{j}$-ésimo bloco. Geralmente os resultados de um experimento com dois fatores são apresentados em uma tabela de $I \times J$ celas contendo n unidades cada uma (ver por exemplo, Guttman, 1982).

O modelo Log-Linear para a distribuição de Birnbaum-Saunders (aditivo) em sua forma geral de um experimento com dois fatores pode ser escrito por,

$$
y_{i j k}=\eta+\tau_{i}+u_{j}+\epsilon_{i j k}
$$

$\operatorname{com} \sum_{i=1}^{I} \tau_{i}=0$ e $\sum_{j=1}^{J} u_{j}=0$, para $-\infty<y_{i j k}<\infty$.

Aqui $y_{i j k}=\ln$ (tempo de sobrevivencia), e $\eta, \underline{I}$ e $\underline{U}$ são os parâmetros do modelo (7.1), com $\underline{I}^{\prime}=\left(\tau_{1}, \ldots, \tau_{I}\right)$ e $\underline{U}^{\prime}=\left(u_{1}, \ldots, u_{J}\right)$, onde $\eta$ é a média geral, $\tau_{i}$ é devido aos diferentes tratamentos (efeito do i-ésimo tratamento), $u_{j}$ é devido aos diferentes blocos (efeito do j-ésimo bloco) e $\epsilon_{i j k}$ são os erros com uma distribuição Senh-Normal com parâmetros $\alpha, \gamma=0$ e $v=2$ (ver densidade (1.25)).

Sejam $y_{111}, \ldots, y_{I J k} \mathrm{n}$ observações independentes do modelo (7.1), com $\epsilon_{i j k}=$ $y_{i j k}-\eta-\tau_{i}-u_{j}$ onde $\epsilon_{i j k} \stackrel{a}{\sim} S N(\alpha, 0,2)$. Então a função de verossimilhança para $\alpha, \eta$, $\underline{I}$ e $\underline{U}$ é dada por,

$$
L(\alpha, \eta, \underline{\tau}, \underline{U})=\left(\frac{1}{2 \sqrt{2 \pi}}\right)^{I J n} \prod_{i=1}^{I} \prod_{j=1}^{J} \prod_{k=1}^{n} W_{i j k} \exp \left\{-\frac{1}{2} \sum_{i=1}^{I} \sum_{j=1}^{J} \sum_{i=1}^{n} Z_{i j k}^{2}\right\}
$$

Por motivos explicados anteriormente, é recomendavel trabalhar com o logaritmo da função de verossimilhança. Assim o logaritmo da função de verossimilhança (da parte informativa) para $\alpha, \eta, \tau$ e $\underline{U}$ é dado por,

$$
l(\alpha, \eta, \underline{\tau}, \underline{U})=\sum_{i=1}^{I} \sum_{j=1}^{J} \sum_{k=1}^{n} \ln W_{i j k}-\frac{1}{2} \sum_{i=1}^{I} \sum_{j=1}^{J} \sum_{k=1}^{n} Z_{i j k}^{2}
$$

onde

$$
\begin{aligned}
& W_{i j k}=\frac{2}{\alpha} \cosh \left[\frac{y_{i j k}-\eta-\tau_{i}-u_{j}}{2}\right], \\
& Z_{i j k}=\frac{2}{\alpha} \operatorname{senh}\left[\frac{y_{i j k}-\eta-\tau_{i}-u_{j}}{2}\right] .
\end{aligned}
$$


Podemos éscrever (7.1) na forma matricial para facilitar os cálculos, como

$$
\underline{y}=P \underline{M}+\underline{\epsilon}
$$

onde

$$
\begin{aligned}
& \underline{\epsilon}=\left(\epsilon_{111}, \ldots, \epsilon_{I J k}\right) \\
& \underline{y}=\left(y_{111}, \ldots, y_{I J k}\right)
\end{aligned}
$$

e

$$
\underline{M}=\left(\eta, \tau_{1}, \ldots, \tau_{I}, u_{1}, \ldots, u_{J}\right)
$$

com

$$
P=\left(\begin{array}{c|cccc|cccc}
1 & 1 & 0 & \cdots & 0 & 1 & 0 & \cdots & 0 \\
1 & 1 & 0 & \cdots & 0 & 0 & 1 & \cdots & 0 \\
. & . & . & \cdots & . & . & . & \cdots & . \\
. & . & . & \cdots & . & . & . & \cdots & . \\
1 & . & . & \cdots & . & . & . & \cdots & . \\
\hline 1 & 0 & 1 & \cdots & 0 & 1 & 0 & \cdots & 0 \\
1 & 0 & 1 & \cdots & 0 & 0 & 1 & \cdots & 0 \\
. & . & . & \cdots & . & . & . & \cdots & . \\
. & . & . & \cdots & . & . & . & \cdots & . \\
. & . & . & \cdots & . & . & . & \cdots & . \\
1 & 0 & 1 & \cdots & 0 & 0 & 0 & \cdots & 1 \\
\hline & & & \vdots & & & & \vdots & \\
\hline 1 & 0 & 0 & \cdots & 1 & 1 & 0 & \cdots & 0 \\
1 & 0 & 0 & \cdots & 1 & 0 & 1 & \cdots & 0 \\
. & . & . & \cdots & . & . & . & \cdots & . \\
. & . & . & \cdots & . & . & . & \cdots & . \\
. & . & . & \cdots & . & . & . & \cdots & . \\
1 & 0 & 0 & \cdots & 1 & 0 & 0 & \cdots & 1
\end{array}\right) .
$$

Observe que o número total de observações é $I \times J$, e $\mathrm{P}$ é uma matriz de ordem $I J \times(I+J+1)$. Para termos P inversivel podemos utilizar o fato de que $\sum_{i=1}^{I} \tau_{i}=$ $\sum_{j=1}^{J} u_{j}=0$, para eliminar os últimos componentes de $\underline{\tau}$ e $\underline{U}$, e definimos os novos parâmetros, pelos vetores,

$$
\underline{\psi}^{\prime}=\left(\eta, \tau_{1}, \ldots, \tau_{I-1}, u_{1}, \ldots, u_{J-1}\right),
$$




$$
\underline{Q}^{\prime}=\left(\alpha, \underline{\psi}^{\prime}\right)
$$

Desta maneira, para todo $(i, j)$ podemos identificar o $(I+J+1)$ vetor $\underline{x}_{i j}$ formado pelos elementos $-1,0$ e 1 , tal que,

$$
\eta+\tau_{i}+u_{j}=\underline{\psi}^{\prime} \underline{x}_{i j}
$$

para $1 \leq i \leq I$ e $1 \leq j \leq J$. Por exemplo, o primeiro elemento do vetor $\underline{x}_{I J}$ é 1 e todos os outros elementos são -1 , como mostramos a seguir,

$$
\underline{x}_{I J}=(1,-1, \cdots,-1,-1, \cdots,-1) \text {. }
$$

Portanto, a nova matriz $\mathrm{P}$ denotada por $X$ será de ordem $I J \times(I+J-1)$ com posto igual a $(I+J-1)$, e dada por,

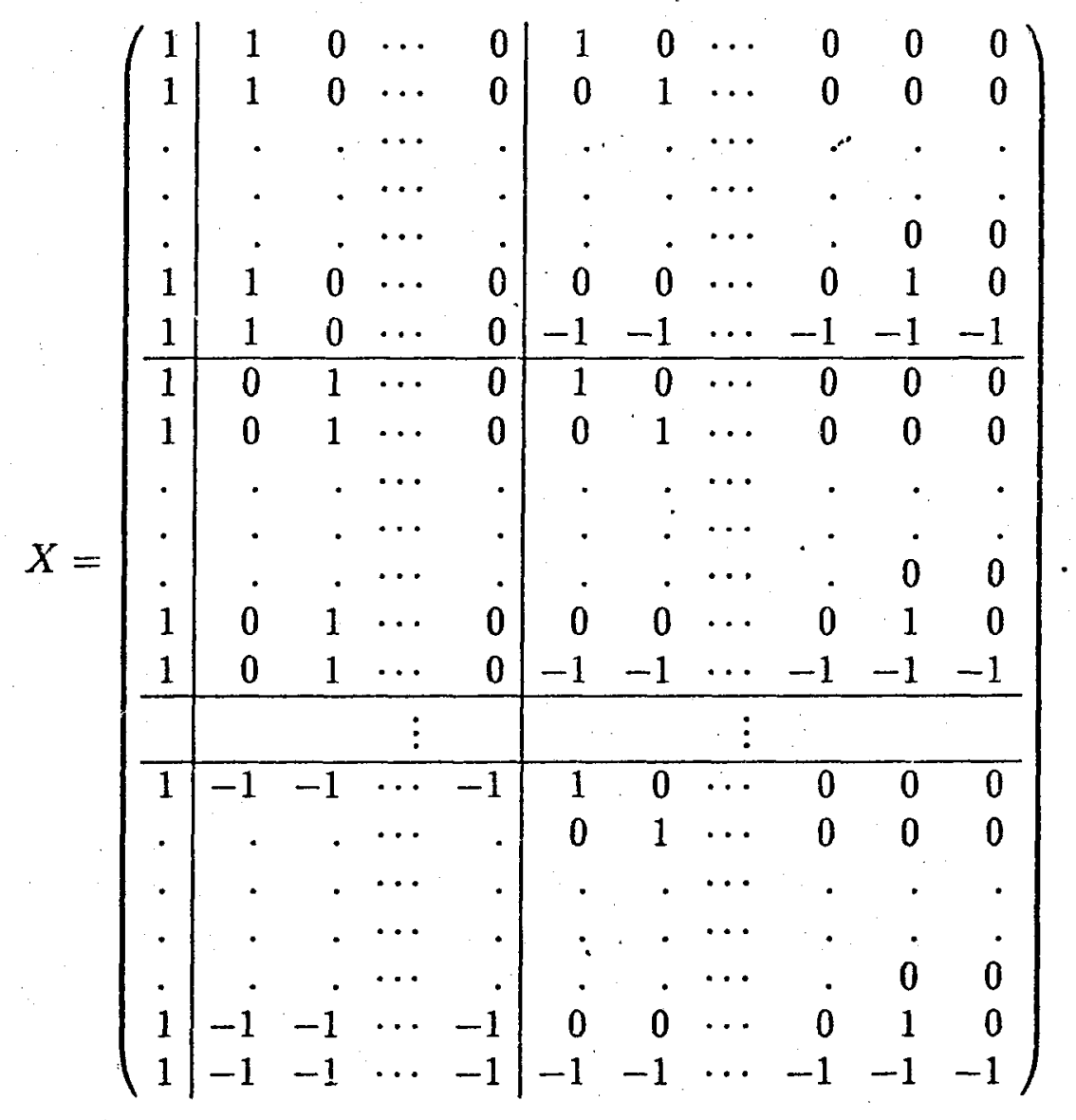


ou seja,

$$
X^{\prime}=\left(\underline{x}_{11}, \underline{x}_{12}, \cdots, \underline{x}_{I J}\right)
$$

Com esta notação, o logaritmo da função de verossimilhança (da parte informativa) da expressão (7.2) é dado por,

$$
l\left(\alpha, \underline{\psi}^{i}\right)=\sum_{i=1}^{I} \sum_{j=1}^{J} \sum_{k=1}^{n} \ln W_{i j k}-\frac{1}{2} \sum_{i=1}^{I} \sum_{j=1}^{J} \sum_{k=1}^{n} Z_{i j k}^{2}
$$

onde

$$
W_{i j k}=\frac{2}{\alpha} \cosh \left[\frac{y_{i j k}-\underline{\psi}^{\prime} \underline{x}_{i j}}{2}\right] \text {, }
$$

e

$$
Z_{i j k}=\frac{2}{\alpha} \operatorname{senh}\left[\frac{y_{i j k}-\underline{\psi}^{\prime} \underline{x}_{i j}}{2}\right] \text {. }
$$

Derivando (7.9) em relação a $\alpha$ e $\underline{\psi}^{\prime}$, obtemos as seguintes equações de verossimilhança:

$$
\begin{gathered}
\frac{\partial l\left(\alpha, \underline{\psi}^{\prime}\right)}{\partial \alpha}=-\frac{I J n}{\alpha}+\frac{1}{\alpha} \sum_{i=1}^{I} \sum_{j=1}^{J} \sum_{k=1}^{n} Z_{i j k}^{2}=0 . \\
\frac{\partial l\left(\alpha, \underline{\psi}^{\prime}\right)}{\partial \underline{\psi}^{\prime}}=\frac{1}{2} \sum_{i=1}^{I} \sum_{j=1}^{J} \sum_{k=1}^{n}\left\{Z_{i j k} W_{i j k}-\frac{Z_{i j k}}{W_{i j k}}\right\}=0
\end{gathered}
$$

Resolvendo a equação (7.10) encontramos uma expressão para o estimador de máxima verossimilhança de $\alpha^{2}$ em termos de $\underline{\hat{\psi}}^{\prime}$, dada por,

$$
\widehat{\alpha}^{2}=\frac{4}{I J n} \sum_{i=1}^{I} \sum_{j=1}^{J} \sum_{k=1}^{n} \operatorname{senh}^{2}\left[\frac{y_{i j k}-\widehat{\psi}^{\prime} \underline{x}_{i j}}{2}\right],
$$

onde $\widehat{\psi}^{\prime}$ é um estimador de máxima verossimilhança de $\underline{\psi}^{\prime}$, o qual é obtido numericamente.

Observe que para n grande podemos fazer inferências sobre os parâmetros $\alpha$ e $\underline{\psi}^{\prime}$, utilizando a aproximação Normal assintótica dos estimadores de máxima verossimilhança $\hat{\alpha}$ e $\widehat{\psi}^{\prime}$. Assim consideramos,

$$
\left(\hat{\alpha}, \hat{\psi}^{\prime}\right) \stackrel{a}{\sim} N\left\{\left(\alpha, \underline{\psi}^{\prime}\right) ; I_{0}^{-1}\right\},
$$

onde $I_{0}$ é a matriz de informação observada. 


\subsubsection{Estimadores de mínimos quadrados.}

Considerar o modelo do tipo,

$$
\bar{y}_{i j}=\eta+\tau_{i}+u_{j}+\epsilon_{i j k}^{*},
$$

para $i=1,2, \ldots, I, j=1,2, \ldots, J$ e $k=1,2, \ldots, n$, onde $\tau_{i}=-\tau_{1}-\ldots-\tau_{I-1}$, $u_{j}=-u_{1}-\ldots-u_{J-1}, \bar{y}_{i j}=\sum_{k=1}^{n} y_{i j} / k$ e $\epsilon_{i j k}^{*}$ são os erros do modelo (7.14) com distribuiçõ normal. Este modelo, também pode-se expressar da seguinte forma:

$$
\underline{y}=X \underline{\psi}+\underline{\epsilon}^{*},
$$

onde

$$
\underline{y}^{\prime}=\left(\bar{y}_{11}, \ldots, \bar{y}_{1 J}, \bar{y}_{21}, \ldots, \bar{y}_{2 J}, \ldots, \bar{y}_{I 1}, \ldots, \bar{y}_{I J}\right),
$$

e

$$
\underline{\epsilon}^{*}=\left(\epsilon_{11}^{*}, \ldots, \epsilon_{1 J}^{*}, \epsilon_{21}^{*}, \ldots, \epsilon_{2 J}^{*}, \ldots, \epsilon_{I 1}^{*}, \ldots, \epsilon_{I J}^{*}\right),
$$

e X representa a matriz (7.S) $\operatorname{com} \underline{\psi}$ dado por (7.6).

Para estimar os parâmetros $\left(\hat{\psi}^{* *}\right)$ do modelo acima, utilizaremos o método de mínimos quadrados. O método de mínimos quadrados consiste em escolher coeficientes $(\underline{\psi})$ de maneira que a soma dos quadrados dos desvios entre os valores observados $\bar{y}_{i j}$ e os valores estimados $\hat{\bar{y}}_{i j}$ seja mínima.

$\mathrm{Na}$ apresentação que segue são omitidas as demonstrações. Para um tratamento formal de modelos lineares, inclusive os de regressão, pode-se ver as notas de Carvalho e Dachs (1982) e os livros de Scheffé (1959) e Searle (1971), entre outros.

Do método de mínimos quadrados, obtemos as seguintes equações (equações normais) dadas por,

$$
\left(X^{\prime} X\right) \hat{\psi}^{* *}=X^{\prime} \underline{y} .
$$

Essas equações podem ser resolvidas facilmente, fornecendo as soluções,

$$
\underline{\psi}^{* *}=\left(X^{\prime} X\right)^{-1} X^{\prime} \underline{y} \text {. }
$$

Os valores ajustados são dados por,

$$
\underline{\hat{y}}=X \underline{\hat{\psi}}^{* *}
$$


Com vetor de erros,

$$
\underline{\hat{\epsilon}}^{*}=\underline{y}-\underline{\hat{y}}
$$

A variancia estimada de $\underline{\psi}$ é,

$$
\operatorname{Var}\left(\underline{\hat{\psi}}^{* *}\right)=\left(X^{\prime} X\right)^{-1} s_{R}^{2}
$$

onde $s_{R}^{2}$ (estimador da $\left.\operatorname{var}\left(\epsilon_{i j}^{*}\right)\right)$ é a soma de quadrados de $(7.19)$ divido por $((I \times J)-$ $(I+J-1))$, isto é

$$
s_{R}^{2}=\frac{\sum_{i=1}^{I} \sum_{j=1}^{J}\left(\bar{y}_{i j}-\hat{\bar{y}}_{i j}\right)^{2}}{((I \times J)-(I+J-1))}
$$

\subsubsection{Estimadores Bayesianos.}

Para uma análise Bayesiana do modelo (7.1) com a notação (7.7) consideraremos uma priori não-informativa para representar o grau de conhecimento sobre os parâmetros $\alpha$ e $\underline{\psi}$. Uma priori não-informativa para $\alpha$ e $\underline{\psi}$, assumindo a independência entre $\alpha$ e $\underline{\psi}$ (ver por exemplo, Box e Tiao, 1973) é dada por,

$$
P(\alpha, \underline{\psi}) \propto \frac{1}{\alpha}
$$

para $\alpha>0,-\infty<\eta, \tau_{i}, u_{j}<\infty, \operatorname{com} i=1,2, \ldots,(I-1), j=1,2, \ldots,(J-1) \mathrm{e}$ $k=1,2, \ldots, n$.

Combinando a função de verossimilhança (7.2) com a densidade a priori (7.22), a densidade a posteriori conjunta para $\alpha$ e $\psi$ é dada por (ver por exemplo, Zellner, 1971),

$$
P(\alpha, \underline{\Psi} / \text { Dados }) \propto \prod_{i=1}^{I} \prod_{j=1}^{J} \prod_{k=1}^{n} W_{i j k} \exp \left\{-\frac{1}{2} \sum_{i=1}^{I} \sum_{j=1}^{J} \sum_{n=1}^{n} Z_{i j k}^{2}\right\} .
$$

para $\alpha>0,-\infty<\eta, \tau_{i}, u_{j}<\infty, \operatorname{com} i=1,2, \ldots, I-1, j=1,2, \ldots, J-1$ e $k=$ $1,2, \ldots, n$, onde $\dot{W}_{i j k}$ e $Z_{i j k}$ são dados por (7.9).

A densidade a posteriori marginal para $\underline{\psi}$ é obtida integrando-se a densidade a posteriori conjunta (7.23) em relação a $\alpha$, a qual pode ser expressa da seguinte forma:

$$
P(\underline{\psi} / \text { Dados }) \propto \prod_{i=1}^{I} \prod_{j=1}^{J} \prod_{k=1}^{n} \cosh \left[\frac{y_{i j k}-\underline{\psi}^{\prime} \underline{x_{i j}}}{2}\right] \int_{0}^{\infty} f(\alpha) d \alpha
$$


onde,

$$
f(\alpha)=\alpha^{-(I J n+1)} \exp \left[\frac{-A(\underline{\psi})}{\alpha^{2}}\right]
$$

e

$$
A(\underline{\psi})=2 \sum_{i=1}^{I} \sum_{j=1}^{J} \sum_{k=1}^{n} \operatorname{senh}^{2}\left[\frac{y_{i j k}-\underline{\psi}^{\prime} \underline{x}_{i j}}{2}\right]
$$

A integral dada na expressão (7.24) pode ser calculada na forma exata, utilizando o resultado (3.1). Portanto usando o resultado (3.1) temos que:

$$
P(\underline{\psi} / D a d o s) \propto \frac{\prod_{i=1}^{I} \prod_{j=1}^{J} \prod_{k=1}^{n} \cosh \left[\frac{y_{i j k}-\underline{\psi}^{\prime} \underline{x}_{i j}}{2}\right]}{\left(\sum_{i=1}^{I} \sum_{j=1}^{J} \sum_{k=1}^{n} \operatorname{senh}^{2}\left[\frac{y_{i j k}-\underline{\underline{\psi}}^{\prime} \underline{\underline{x}}_{i j}}{2}\right]\right)^{\frac{I J \underline{n}}{2}}},
$$

para $-\infty<\eta, \tau_{i}, u_{j}<\infty, i=1,2, \ldots, I-1$ e $j=1,2, \ldots, J-1$.

\subsection{Um caso especial do modelo.}

\subsubsection{Estimadores de mínimos quadrados.}

Supondo que temos uma variável tratamento A com 2 níveis $(I=2)$ e uma variável bloco $\mathrm{B}$ com 2 niveis $(J=2)$, os resultados de um experimento com dois fatores são apresentados em uma tábua de $2 \times 2$ celas contendo $n$ unidades, como se mostra na tabela 7.1.

Tabela 7.1 Apresentação tabular dos resultados dos resultados de um experimento com dois fatores.

\begin{tabular}{c|c|c|c}
\hline \multirow{2}{*}{} & \multicolumn{3}{|c}{ Tratamento. } \\
\cline { 2 - 3 } & 1 & 2 \\
\hline Bloco. & 1 & $y_{111}, \ldots, y_{11 n}$ & $y_{211}, \ldots, y_{21 n}$ \\
\cline { 2 - 3 } & 2 & $y_{121}, \ldots, y_{12 n}$ & $y_{221}, \ldots, y_{22 n}$ \\
\hline
\end{tabular}


De (7.14) temos que,

$$
\bar{y}_{i j}=\eta+r_{i}+u_{j}+\epsilon_{i j k}^{*}
$$

para $i=1,2$ e $j=1,2$, onde,

$$
\left\{\begin{array}{l}
\tau_{1}+\tau_{2}=0 \\
u_{1}+u_{2}=0
\end{array}\right.
$$

isto implica que,

$$
\left\{\begin{array}{l}
\tau_{2}=-\tau_{1} \\
u_{2}=-u_{1}
\end{array}\right.
$$

$(7.15))$

$\mathrm{O}$ modelo (7.26) pode ser expresso matricialmente da seguinte forma (ver

$$
\left[\begin{array}{l}
\bar{y}_{11} \\
\bar{y}_{12} \\
\bar{y}_{21} \\
\bar{y}_{22}
\end{array}\right]=\left[\begin{array}{ccc}
1 & 1 & 1 \\
1 & 1 & -1 \\
1 & -1 & 1 \\
1 & -1 & -1
\end{array}\right] \times\left[\begin{array}{c}
\eta \\
\tau_{1} \\
u_{1}
\end{array}\right]+\left[\begin{array}{c}
\epsilon_{11}^{*} \\
\epsilon_{12}^{* *} \\
\epsilon_{21}^{*} \\
\epsilon_{22}^{*}
\end{array}\right]
$$

Para resolver as equações normais (7.16), temos que calcular as matrizes $\left(X^{\prime} X\right),\left(X^{\prime} X\right)^{-1}$ e $X^{\prime} \underline{y}$, as quais são dadas por,

$$
\begin{gathered}
X^{\prime} X=\left(\begin{array}{ccc}
4 & 0 & 0 \\
0 & 4 & 0 \\
0 & 0 & 4
\end{array}\right), \\
\left(X^{\prime} X\right)^{-1}=\left(\begin{array}{ccc}
\frac{1}{4} & 0 & 0 \\
0 & \frac{1}{4} & 0 \\
0 & 0 & \frac{1}{4}
\end{array}\right),
\end{gathered}
$$




$$
X^{\prime} \underline{\underline{y}}=\left(\begin{array}{l}
\sum_{i=1}^{2} \sum_{j=1}^{2} \bar{y}_{i j} . \\
\sum_{j=1}^{2} \bar{y}_{1 j}-\sum_{j=1}^{2} \bar{y}_{2 j} \\
\bar{y}_{11}-\bar{y}_{12}-\bar{y}_{21}-\bar{y}_{22}
\end{array}\right) .
$$

Portanto utilizando (7.17) temos que os EMQ (estimadores de mínimos quadrados), para $\eta, \tau_{1}$ e $u_{1}$ são, dados por,

$$
\left\{\begin{array}{l}
\hat{\eta}^{* *}=\frac{1}{4} \sum_{i=1}^{2} \sum_{j=1}^{2} \bar{y}_{i j} \\
\widehat{\tau}_{1}^{* *}=\frac{1}{4} \sum_{j=1}^{2} \bar{y}_{1 j}-\frac{1}{4} \sum_{j=1}^{2} \bar{y}_{2 j} \\
\widehat{u}_{1}^{* *}=\frac{1}{4}\left(\bar{y}_{11}-\bar{y}_{12}-\bar{y}_{21}-\bar{y}_{22}\right) .
\end{array}\right.
$$

onde

A variancia estimada de $\underline{\psi}$ pode ser calçulada utilizando a expressão (7.20),

$$
s_{R}^{2}=\sum_{i=1}^{2} \sum_{j=1}^{2}\left(\bar{y}_{i j}-\hat{\bar{y}}_{i j}\right)^{2}
$$

observe que $I=2$ e $J=2$, por conseguinte $((I \times J-(I+J-1))=((2 \times 2)-(2+2-1))=$ $4-3=1$.

\subsubsection{Estimadores de máxima verossimilhança.}

Usualmente nosso objetivo é obter inferências a respeito dos parâmetros. Um estimador muito utilizado devido a suas boas propriedades é dado pelo estimador de máxima veiossimilhança. Por motivos explicados em capítulos anteriores, é recomendavel trabalhar com o logaritmo da função de verossimilhança. Assim o logaritmo da função de verossimilhança (da parte informativa) para $\underline{\psi}$ e $\alpha$ (por (7.9)) é,

$$
l\left(\underline{\psi}^{\prime}, \alpha\right)=\sum_{i=1}^{2} \sum_{j=1}^{2} \sum_{k=1}^{n} \ln W_{i j k}-\frac{1}{2} \sum_{i=1}^{2} \sum_{j=1}^{2} \sum_{k=1}^{n} Z_{i j k}^{2}
$$

onde

$$
W_{i j k}=\frac{2}{\alpha} \cosh \left[\frac{y_{i j k}-\underline{\psi}^{\prime} \underline{x}_{i j}}{2}\right]
$$




$$
Z_{i j k}=\frac{2}{\alpha} \operatorname{senh}\left[\frac{y_{i j k}-\underline{\psi}^{\prime} \underline{x}_{i j}}{2}\right] .
$$

As primeiras derivadas de (7.35) em relação a $\alpha$ e $\underline{\psi}^{\prime}$ são dadas por,

$$
\begin{gathered}
\frac{\partial l\left(\alpha, \underline{\left.\psi^{\prime}\right)}\right.}{\partial \alpha}=-\frac{4 n}{\alpha}+\frac{1}{\alpha} \sum_{j=1}^{2} \sum_{j=1}^{2} \sum_{k=1}^{n} Z_{i j k}^{2}=0 . \\
\frac{\partial l\left(\alpha, \underline{\psi}^{\prime}\right)}{\partial \eta}=\frac{1}{2} \sum_{i=1}^{2} \sum_{j=1}^{2} \sum_{k=1}^{n}\left\{Z_{i j k} W_{i j k}-\frac{Z_{i j k}}{W_{i j k}}\right\}=0 \\
\frac{\partial l\left(\alpha, \underline{\psi}^{\prime}\right)}{\partial \tau_{1}}=\frac{1}{2} \sum_{i=1}^{2} \sum_{j=1}^{2} \sum_{k=1}^{n}(-1)^{i}\left\{Z_{i j k} W_{i j k}-\frac{Z_{i j k}}{W_{i j k}}\right\}=0 \\
\frac{\partial l\left(\alpha, \underline{\psi}^{\prime}\right)}{\partial u_{1}}=\frac{1}{2} \sum_{i=1}^{2} \sum_{j=1}^{2} \sum_{k=1}^{n}(-1)^{j}\left\{Z_{i j k} W_{i j k}-\frac{Z_{i j k}}{W_{i j k}}\right\}=0 .
\end{gathered}
$$

Portanto resolvendo a equação (7.36) encontramos uma expressão para o estimador de máxima verossimilhança de $\alpha^{2}$ em termos de $\widehat{\underline{\psi}}^{\prime}\left(\widehat{\eta}, \widehat{\tau}_{1}, \widehat{u}_{1}\right)$, a qual é dada por:

$$
\widehat{\alpha}^{2}=\frac{1}{n} \sum_{i=1}^{2} \sum_{j=1}^{2} \sum_{k=1}^{n} \operatorname{senh}^{2}\left[\frac{y_{i j k}-\widehat{\psi}^{\prime} \underline{x}_{i j}}{2}\right],
$$

onde $\hat{\eta}, \widehat{\tau}_{1}, \widehat{u}_{1}$ são estimadores de máxima verossimilhança de $\eta, \tau_{1}$ e $u_{1}$ os quais são obtidos numericamente, utilizando (7.37), (7.38) e (7.39). iterativo.

Nota: Podemos utilizar os EMQ como estimadores iniciais para o método

\subsubsection{Inferências sobre os parâmetros $\alpha, \eta, \tau_{1}$ e $u_{1}$.}

Para $\mathrm{n}$ grande podemos fazer inferências sobre os parâmetros $\psi^{\prime}$ e $\alpha$, utilizando a aproximação Normal assintótica dos estimadores de máxima verossimilhança $\hat{\eta}, \widehat{\tau}_{1}, \widehat{u}_{1}$ e $\hat{\alpha}$. Assim consideramos,

$$
\left(\widehat{\eta}, \widehat{\tau}_{1}, \widehat{u}_{1}, \widehat{\alpha}\right) \stackrel{a}{\sim} N\left\{\left(\eta, \tau_{1}, u_{1}, \alpha\right) ; I_{0}^{-1}\right\}
$$


onde $I_{0}$ é a matriz de informação observada, dada por:

$$
I_{0}=\left(\begin{array}{llll}
-\frac{1}{4} \widehat{Q}_{0} & \frac{1}{4} \hat{Q}_{i} & \frac{1}{4} \hat{Q}_{j} & \frac{1}{\alpha} Q \widehat{W} Z_{0} \\
\frac{1}{4} \widehat{Q}_{i} & -\frac{1}{4} \widehat{Q}_{0} & -\frac{1}{4} \hat{Q}_{i+j} & -\frac{1}{\alpha} Q \widehat{W} Z_{i} \\
\frac{1}{4} \widehat{Q}_{j} & -\frac{1}{4} \hat{Q}_{i+j} & -\frac{1}{4} \hat{Q}_{0} & -\frac{1}{\alpha} Q \widehat{W} Z_{j} \\
\frac{1}{\alpha} Q \widehat{W} Z_{0} & -\frac{1}{\alpha} Q \widehat{W} Z_{i} & -\frac{1}{\alpha} Q \widehat{W} Z_{j} & -\frac{1}{\alpha^{2}} \widehat{Q Z}
\end{array}\right),
$$

onde

$$
\widehat{W}_{i j k}=\cosh ^{2}\left[\frac{y_{i j k}-\widehat{\underline{\psi}}^{\prime} \underline{x}_{i j}}{2}\right]
$$

e

$$
\hat{Z}_{i j k}=\operatorname{senh}^{2}\left[\frac{y_{i j k}-\widehat{\underline{\psi}}^{\prime} \underline{x}_{i j}}{2}\right],
$$

com

$$
\begin{gathered}
\hat{Q}_{l}=\sum_{i=1}^{2} \sum_{j=1}^{2} \sum_{k=1}^{n}(-1)^{l}\left[1-\widehat{Z}_{i j k}^{2}-\widehat{W}_{i j k}^{2}-\frac{\widehat{Z}_{i j k}^{2}}{\widehat{W}_{i j k}^{2}}\right] \\
\widehat{Q W} Z_{l}=\sum_{i=1}^{2} \sum_{j=1}^{2} \sum_{k=1}^{n}(-1)^{l} \widehat{W}_{i j k} \widehat{Z}_{i j k} \\
\widehat{Q Z}=3 \sum_{i=1}^{2} \sum_{j=1}^{2} \sum_{k=1}^{n} \widehat{Z}_{i j k}^{2}-4 n,
\end{gathered}
$$

para $l=0, i, j, i+j$, onde $\widehat{\psi}^{\prime}$ é um estimador de máxima verossimilhança de $\underline{\psi}^{\prime}$, o qual é obtido numericamente, para $i=1,2 ; j=1,2$ e $k=1, \ldots, n$.

Os elementos de (7.42) são as segundas derivadas parciais estimadas de (7.35) em relação a $\eta, \tau_{1}, u_{1}$ e $\alpha$ a menos do sinal, dadas por,

$$
\frac{\partial^{2} l\left(\eta, \tau_{1}, u_{1}, \alpha\right)}{\partial \eta^{2}}=\frac{1}{4} \sum_{i=1}^{2} \sum_{j=1}^{2} \sum_{k=1}^{n}\left[1-Z_{i j k}^{2}-W_{i j k}^{2}-\frac{Z_{i j k}^{2}}{W_{i j k}^{2}}\right],
$$




$$
\begin{gathered}
\frac{\partial^{2} l\left(\eta, \tau_{1}, u_{1}, \alpha\right)}{\partial \eta \partial \tau_{1}}=-\frac{1}{4} \sum_{i=1}^{2} \sum_{j=1}^{2} \sum_{k=1}^{n}(-1)^{i}\left[1-Z_{i j k}^{2}-W_{i j k}^{2}-\frac{Z_{i j k}^{2}}{W_{i j k}^{2}}\right] \\
\frac{\partial^{2} l\left(\eta, \tau_{1}, u_{1}, \alpha\right)}{\partial \eta \partial u_{1}}=-\frac{1}{4} \sum_{i=1}^{2} \sum_{j=1}^{2} \sum_{k=1}^{n}(-1)^{j}\left[1-Z_{i j k}^{2}-W_{i j k}^{2}-\frac{Z_{i j k}^{2}}{W_{i j k}^{2}}\right] \\
\frac{\partial^{2} l\left(\eta, \tau_{1}, u_{1}, \alpha\right)}{\partial \eta \partial \alpha}=-\frac{1}{\alpha} \sum_{i=1}^{2} \sum_{j=1}^{2} \sum_{k=1}^{n} W_{i j k} Z_{i j k} \\
\frac{\partial^{2} l\left(\eta, \tau_{1}, u_{1}, \alpha\right)}{\partial \tau_{1}^{2}}=\frac{1}{4} \sum_{i=1}^{2} \sum_{j=1}^{2} \sum_{k=1}^{n}\left[1-Z_{i j k}^{2}-W_{i j k}^{2}-\frac{Z_{i j k}^{2}}{W_{i j k}^{2}}\right] \\
\frac{\partial^{2} l\left(\eta, \tau_{1}, u_{1}, \alpha\right)}{\partial \tau_{1} \partial u_{1}}=\frac{1}{4} \sum_{i=1}^{2} \sum_{j=1}^{2} \sum_{k=1}^{n}(-1)^{i+j}\left[1-Z_{i j k}^{2}-W_{i j k}^{2}-\frac{Z_{i j k}^{2}}{W_{i j k}^{2}}\right] \\
\frac{\partial^{2} l\left(\eta, \tau_{1}, u_{1}, \alpha\right)}{\partial \tau_{1} \partial \alpha}=\frac{1}{\alpha} \sum_{i=1}^{2} \sum_{j=1}^{2} \sum_{k=1}^{n}(-1)^{i} W_{i j k} Z_{i j k}, \\
\frac{\partial^{2} l\left(\eta, \tau_{1}, u_{1}, \alpha\right)}{\partial u_{1}^{2}}=\frac{1}{4} \sum_{i=1}^{2} \sum_{j=1}^{2} \sum_{k=1}^{n}\left[1-Z_{i j k}^{2}-W_{i j k}^{2}-\frac{Z_{i j k}^{2}}{W_{i j k}^{2}}\right] \\
\frac{\partial^{2} l\left(\eta, \tau_{1}, u_{1}, \alpha\right)}{\partial u_{1} \partial \alpha}=\frac{1}{\alpha} \sum_{i=1}^{2} \sum_{j=1}^{2} \sum_{k=1}^{n}(-1)^{j} W_{i j k} Z_{i j k}, \\
\frac{\partial^{2} l\left(\eta, \tau_{1}, u_{1}, \alpha\right)}{\partial \alpha^{2}}=\frac{4 n}{\alpha^{2}}-\frac{3}{\alpha^{2}} \sum_{i=1}^{2} \sum_{j=1}^{2} \sum_{k=1}^{n} Z_{i j k}^{2} .
\end{gathered}
$$

\subsubsection{Estimadores Bayesianos.}

para $\underline{\psi} \dot{e}$,

Da expressão (7.25) nos temos que a densidade a posteriori marginal conjunta

$$
\begin{aligned}
& P(\underline{\psi} / \text { Dados }) \propto \frac{\prod_{i=1}^{2} \prod_{j=1}^{2} \prod_{k=1}^{n} \cosh \left[\frac{\left.y_{i j k}-\underline{\psi}^{\prime} \underline{x}_{i j}\right]}{2}\right]}{\left(\sum_{i=1}^{2} \sum_{j=1}^{2} \sum_{k=1}^{n} \operatorname{senh}^{2}\left[\frac{y_{i j k}-\underline{\psi}^{\prime} \underline{x}_{i j}}{2}\right]\right)^{2 n}}, \\
& \text { para }-\infty<\eta, \tau_{1}, u_{1}<\infty, I=J=2 .
\end{aligned}
$$

Observar que (7.53), pode ser expressa da seguinte forma, 


$$
P\left(\eta, \tau_{1}, u_{1} / D a d o s\right) \propto \frac{\prod_{i=1}^{2} \prod_{j=1}^{2} \prod_{k=1}^{n} M_{i j k}}{\left(\sum_{i=1}^{2} \sum_{j=1}^{2} \sum_{k=1}^{n} N_{i j k}^{2}\right)^{2 n}},
$$

para $-\infty<\eta, \tau_{1}, u_{1}<\infty$, onde

$$
M_{i j k}=\cosh \left[\frac{y_{i j k}-\underline{\psi}^{\prime} \underline{x}_{i j}}{2}\right],
$$

e

$$
N_{i j k}=\operatorname{senh}\left[\frac{y_{i j k}-\underline{\psi}^{\prime} \underline{x}_{i j}}{2}\right] \text {. }
$$

Para calcular a moda basta derivar o logaritmo de (7.54) em relação a $\eta, \tau_{1} \mathrm{e}$ $u_{1}$, igualar a zero e resolver. Então,

$$
\ln P\left(\eta, \tau_{1}, u_{1}\right) \propto \sum_{i=1}^{2} \sum_{j=1}^{2} \sum_{k=1}^{n} \ln M_{i j k}-2 n \times \ln \left(\sum_{i=1}^{2} \sum_{j=1}^{2} \sum_{k=1}^{n} N_{i j k}^{2}\right) .
$$

Portanto,

$$
\begin{aligned}
& \frac{\partial l\left(\eta, \tau_{1}, u_{1}\right)}{\partial \eta}=-\frac{1}{2} \sum_{i=1}^{2} \sum_{j=1}^{2} \sum_{k=1}^{n} \frac{N_{i j k}}{M_{i j k}}+n \times \frac{\sum_{i=1}^{2} \sum_{j=1}^{2} \sum_{k=1}^{n} 2 N_{i j k} M_{i j k}}{\sum_{i=1}^{2} \sum_{j=1}^{2} \sum_{k=1}^{n} N_{i j k}^{2}}, \\
& \frac{\partial l\left(\eta, \tau_{1}, u_{1}\right)}{\partial \tau_{1}}=\frac{1}{2} \sum_{i=1}^{2} \sum_{j=1}^{2} \sum_{k=1}^{n}(-1)^{i} \frac{N_{i j k}}{M_{i j k}}-n \times \frac{\sum_{i=1}^{2} \sum_{j=1}^{2} \sum_{k=1}^{n}(-1)^{i} 2 N_{i j k} M_{i j k}}{\sum_{i=1}^{2} \sum_{j=1}^{2} \sum_{k=1}^{n} N_{i j k}^{2}}, \\
& \frac{\partial l\left(\eta, \tau_{1}, u_{1}\right)}{\partial u_{1}}=\frac{1}{2} \sum_{i=1}^{2} \sum_{j=1}^{2} \sum_{k=1}^{n}(-1)^{j} \frac{N_{i j k}}{M_{i j k}}-n \times \frac{\sum_{i=1}^{2} \sum_{j=1}^{2} \sum_{k=1}^{n}(-1)^{j} 2 N_{i j k} M_{i j k}}{\sum_{i=1}^{2} \sum_{j=1}^{2} \sum_{k=1}^{n} N_{i j k}^{2}} .
\end{aligned}
$$

Assim, igualando (7.56), (7.57) e (7.58) a zero encontramos a moda da a posteriori marginal para $\underline{\hat{\psi}}^{*}$, dada por,

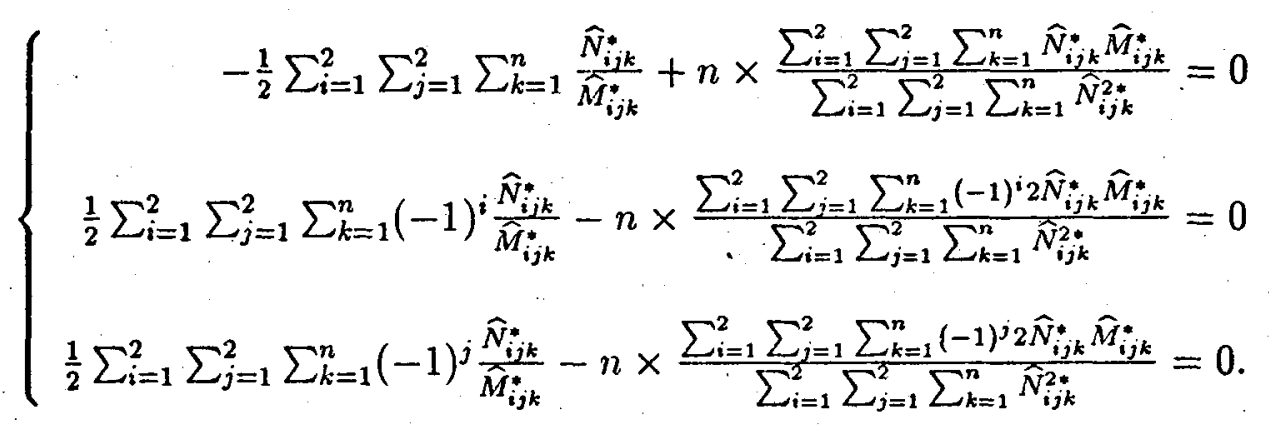


Com

$$
\widehat{M}_{i j k}^{*}=\cosh \left[\frac{y_{i j k}-\widehat{\hat{\psi}}^{*} \underline{x}_{i j}}{2}\right] \text {, }
$$

e

$$
\widehat{N}_{i j k}^{*}=\operatorname{senh}\left[\frac{y_{i j k}-\widehat{\psi}^{*} \underline{x}_{i j}}{2}\right],
$$

onde $\widehat{\psi}^{*}$ é um estimador bayesiano de $\underline{\psi}^{\prime}$, o qual é obtido numericamente, para $i=1,2$; $j=1, \overline{2}$ e $k=1, \ldots, n$. 


\section{Capítulo 8}

\section{Três exemplos de aplicação}

Neste capítulo, apresentamos três exemplos de aplicação dos procedimentos obtidos nos capítulos anteriores. Assim, realizaremos uma análise clássica e Bayesiana para o modelo de Birnbaum-Saunders (exemplo 1), para o modelo Log-Linear da distribuição de Birnbaum-Saunders com uma variável stress (exemplo 2) e para um planejamento de experimentos (exemplo 3).

\subsection{Exemplo 1.}

\subsubsection{Descrição dos dados.}

$\mathrm{Na}$ tabela 8.1 , temos tempos de sobrevivência em ciclos $\times 10^{-3}$ para 101 amostras (láminas) em stress máximo (stress máximo ou valor máximo da tensão, genericamente é a resistência suportada por um material á fadiga, para um dado número de ciclos, sem romper) por ciclos de 31000 PSI (libras/polegadas quadradas). Este conjunto de dados foi também considerado por Birnbaum e Saunders (1969b).

Os dados em estudo são tempos de fadiga de amostras de componentes de aluminio 6061-T6, com cortes do tipo paralelo com cisalhamento por flexão rotativa e oscilações de 18 ciclos por segundo. 
Tabela 8.1 Tempos de sobrevivência em ciclos $\times 10^{-3}$ para 101. componentes em stress máximo por ciclos de 31000 polegadas quadradas.

\begin{tabular}{rrrrrrrr}
\hline 70 & 90 & 96 & 97 & 99 & 100 & 103 & 104 \\
104 & 105 & 107 & 108 & 108 & 108 & 109 & 109 \\
112 & 112 & 113 & 114 & 114 & 114 & 116 & 119 \\
120 & 120 & 120 & 121 & 121 & 123 & 124 & 124 \\
124 & 124 & 124 & 128 & 128 & 129 & 129 & 130 \\
130 & 130 & 131 & 131 & 131 & 131 & 131 & 132 \\
132 & 132 & 133 & 134 & 134 & 134 & 134 & 134 \\
136 & 136 & 137 & 138 & 138 & 138 & 139 & 139 \\
141 & 141 & 142 & 142 & 142 & 142 & 142 & 142 \\
144 & 144 & 145 & 146 & 148 & 148 & 149 & 151 \\
151 & 152 & 155 & 156 & 157 & 157 & 157 & 157 \\
158 & 159 & 162 & 163 & 163 & 164 & 166 & 166 \\
168 & 170 & 174 & 196 & 212 & & & \\
\hline
\end{tabular}

\subsubsection{Verificação gráfica da adequabilidade do modelo.}

Um procedimento gráfico pode ser utilizado para verificar a adequabilidade da distribuição de Birnbaum-Saunders. Para tal propósito considere a função de distribuição acumulada $F(\hat{\alpha}, \widehat{\beta})$ dada por (1.11), onde $Z$ expressa por (1.12) é normalmente distribuida com média zero e variancia 1 , e $\widehat{\alpha}$ e $\widehat{\beta}$ são os estimadores de máxima verossimilhança de $\alpha$ e $\beta$ (ver, Birnbaum e Saunders, 1969b). Por conseguinte temos que verificar se os dados seguem uma distribuição normal. Uma das ferramentas mais apropriadas para avaliar a suposição de normalidade para os dados de forma satisfatória são os chamados gráficos probabilísticos normais.

O gráfico probabilístico normal consiste de uma figura na qual, se os valores forem "exatamente normais", os pontos correspondentes deveriam ficar exatamente sobre uma reta. É claro que mesmo em amostras de uma normal, devido ás flutações, os pontos oscilam em torno da reta. Para entender o processo de construção desse gráfico considerese a densidade normal $(0,1)$ e sua distribuição cumulativa dada por (1.9). Se os dados observados são de uma normal $(0,1)$ então sua distribuição cumulativa empírica devería estar próxima da cumulativa normal. Desta maneira obtemos a tabela 8.11 (ver apêndice B). Na figura 8.1 (utilizando os valores da tabela 8.11), estão representados os 101 valores $\left(t_{i}\right)$, sua distribuição cumulativa empírica $(1 / \mathrm{i})$ e a cumulativa $F(\widehat{\alpha}, \widehat{\beta})$. 


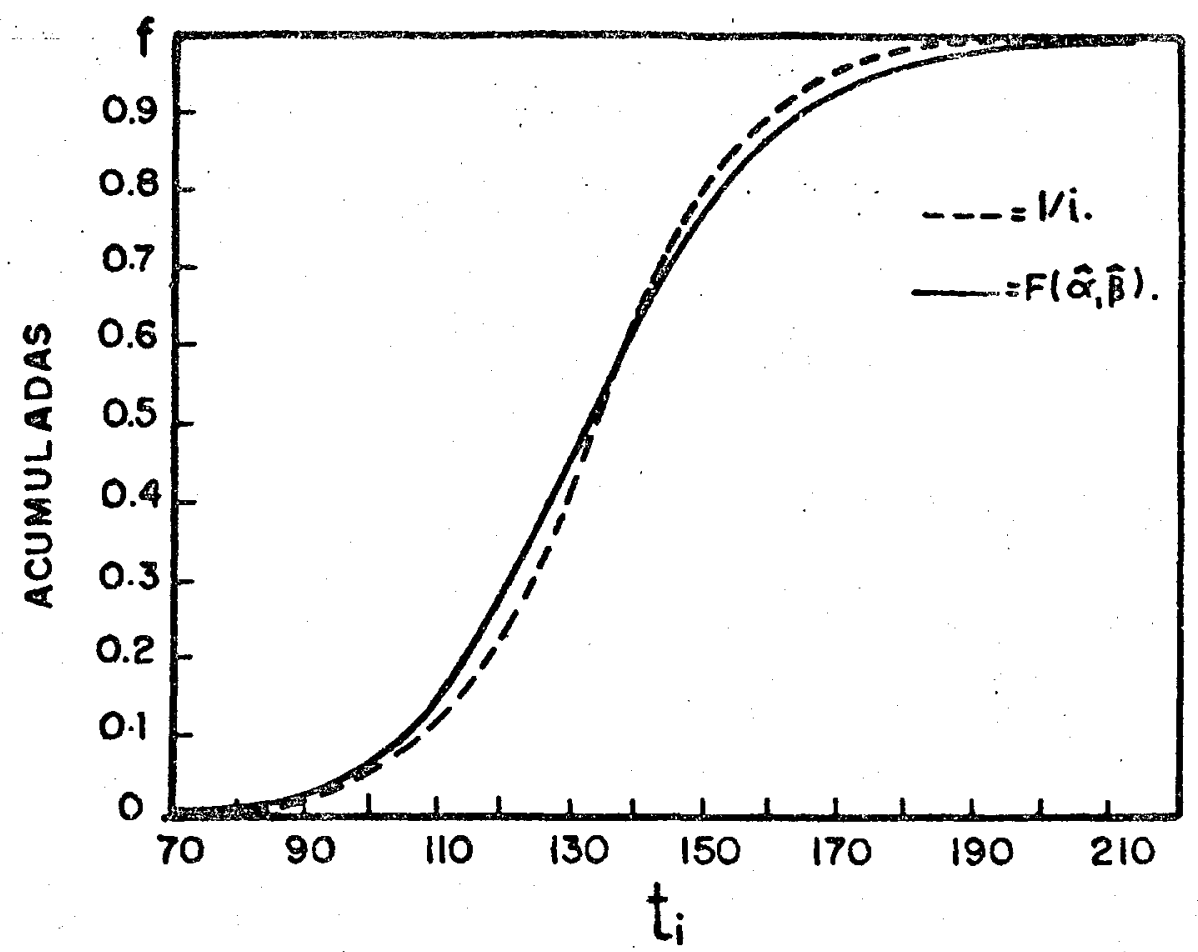

Figura 8.1 Comparação gráfica da distribuição empírica acumulada e a distribuição $F(\hat{\alpha}, \widehat{\beta})$, para os dados da tabela 8.1 .

Da comparação gráfica (na figura 8.1), podemos concluir que os dados da tabela 8.1 são adequados para a distribuição de Birnbaum-Saunders.

Como é muito difícil, visualmente, perceber afastamentos sistemáticos, ou excepcionalmente aberrantes, principalmente nas caudas da distribuição, é recomendavel colocar num grafico os valores de $t_{i}$ versus os $t_{i}$ padronizados (NSCORES), para $1 \leq$ $i \leq n$, isto é o grafico dos pares de valores $\left(t_{i}, \mathrm{NSCORES}\right)$. Se esses valores são realmente provenientes de uma amostra normal, os pontos correspondentes devem ficar praticamente sobre uma reta. Para calcular os NSCORES utilizar $F\left(t_{i}\right)=(i-3 / 8) /(n+1 / 4)$, para $1 \leq i \leq n$, e ver seu respectivo valor de $Z$, na tabela da normal (NSCORES). Na figura 8.2, utilizando os valores da tabela 8.11 construimos o gráfico dos NSCORES versus $t_{i}$.

Da figura 8.2 podemos observar à existência de uma relação linear aproximada entre NSCORES e os $t_{i}$. Isto implica na adequabilidade do modelo da distribuição de Birnbaum-Saunders para os dados da tabela 8.1. Observe a presença de 3 pontos um pouco afastados da reta, os quais não tinham sido detectados no gráfico da figura 8.1, porem não considerados problemáticos. 


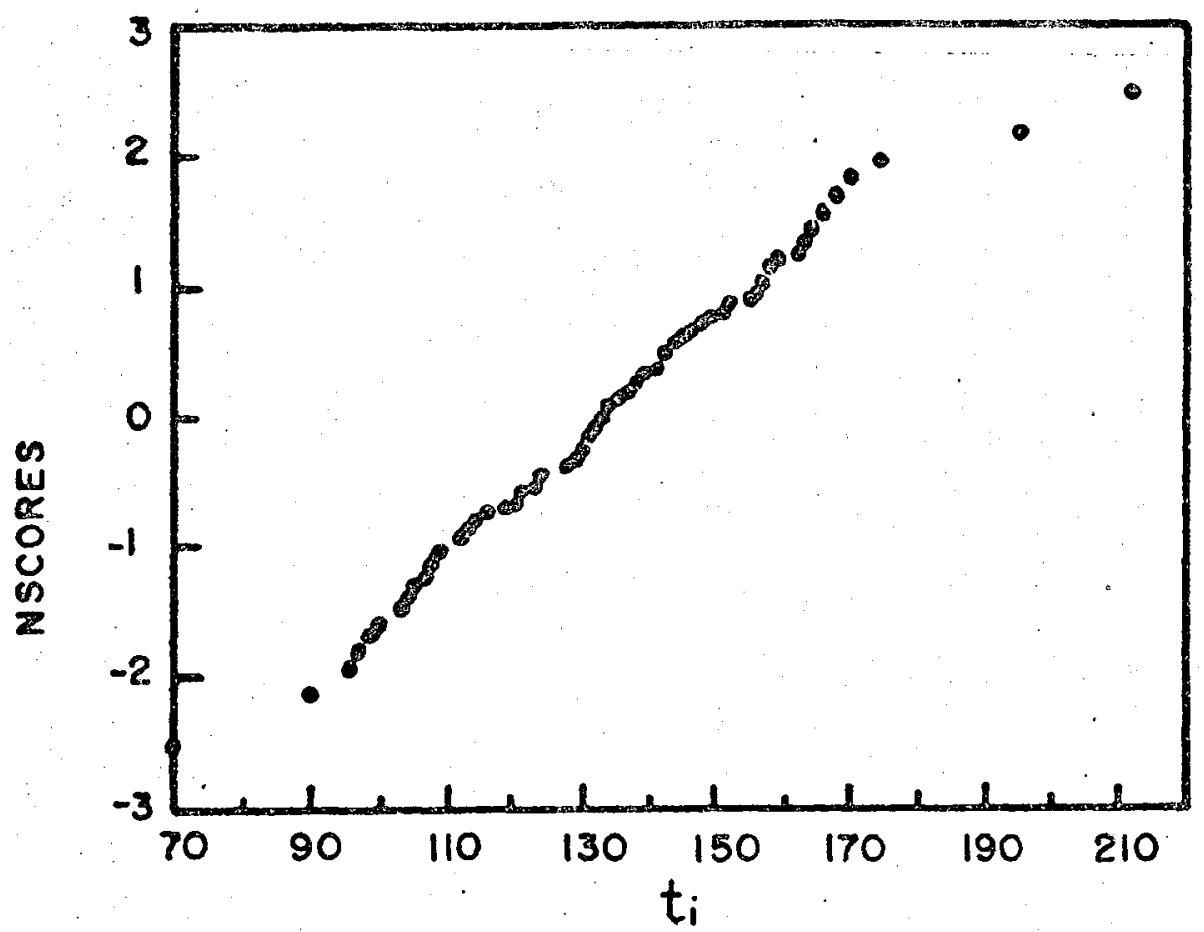

Figura 8.2 Gráfico dos NSCORES versus ciclos $t_{i}$.

\subsubsection{Análise clássica do problema.}

Utilizando a metodología obtida no capítulo 2 reaiizaremos uma análise clássica, dos dados da tabela 8.1. Nesta tabela temos $n=101, S=133.73$ e $R=129.93$ (S e $\mathrm{R}$ são dados em (2.1)). Os estimadores de máxima verossimilhança para $\alpha$ e $\beta$ obtidos numéricamente são dados por $\widehat{\alpha}=0.1704$ e $\widehat{\beta}=131.82$, isto empregando as expressões (2.7) e (2.10).

Considerando a aproximação Normal assintótica dos estimadores de máxima verossimilhança $\hat{\alpha}$ e $\widehat{\beta}$ dada em (2.11), e utilizando a matriz de informação de Fisher em sua forma simplificada (2.21), os intervalos de confiança $90 \%$ para $\alpha$ e $\beta$ são $(0.1506 ; 0.1902)$ e $(128.14 ; 135.49)$ respectivamente. Utilizando integração numérica para calcular $E(t+\beta)^{-2}$ na informação de Fisher (2.17), o intervalo de confiança $90 \%$ para $\beta$ é $(128.15 ; 135.49)$.

Observe que: $E(t+\beta)^{-2}=0.0000145$ (utilizando integração numérica (regra de SIMPSON)), e $E(t+\beta)^{-2} \simeq 0.0000144$ (utilizando a aproximação de LAPLACE).

$O$ estimador de máxima verossimilhança para o tempo médio de vida $\lambda^{*}=$ $\beta\left(1+\frac{\alpha^{2}}{2}\right)$ considerando os estimadores de máxima verossimilhança $\widehat{\alpha}$ e $\widehat{\beta}$, é dado por $\widehat{\lambda}^{*}=$ 
133.73 e um intervalo de confiança aproximado a $90 \%$ para $\lambda^{*}$ (ver, 2.26) é $(129.97 ; 137.48)$.

\subsubsection{Análise Bayesiana do exemplo 1 , assumindo $\alpha$ e $\beta$ des- conhecidos.}

Em inferência Bayesiana, as inferencias sobre os parâmetros de interesse são realizadas utilizando a suas densidades a posteriori. Assim considerando os dados da tabela 8.1, construimos (ver figura 8.3) os gráficos das densidades a posteriori marginais para $\alpha(3.7)$ e (3.8), considerando as priori não informativas (3.2) e (3.4), obtidas pelo método de aproximação de LAPLACE. As modas destas densidades a posteriori marginais são dadas por $\hat{\alpha}^{*} \simeq 0.1696$ e $\hat{\alpha}^{*} \simeq 0.1704$, respectivamente.

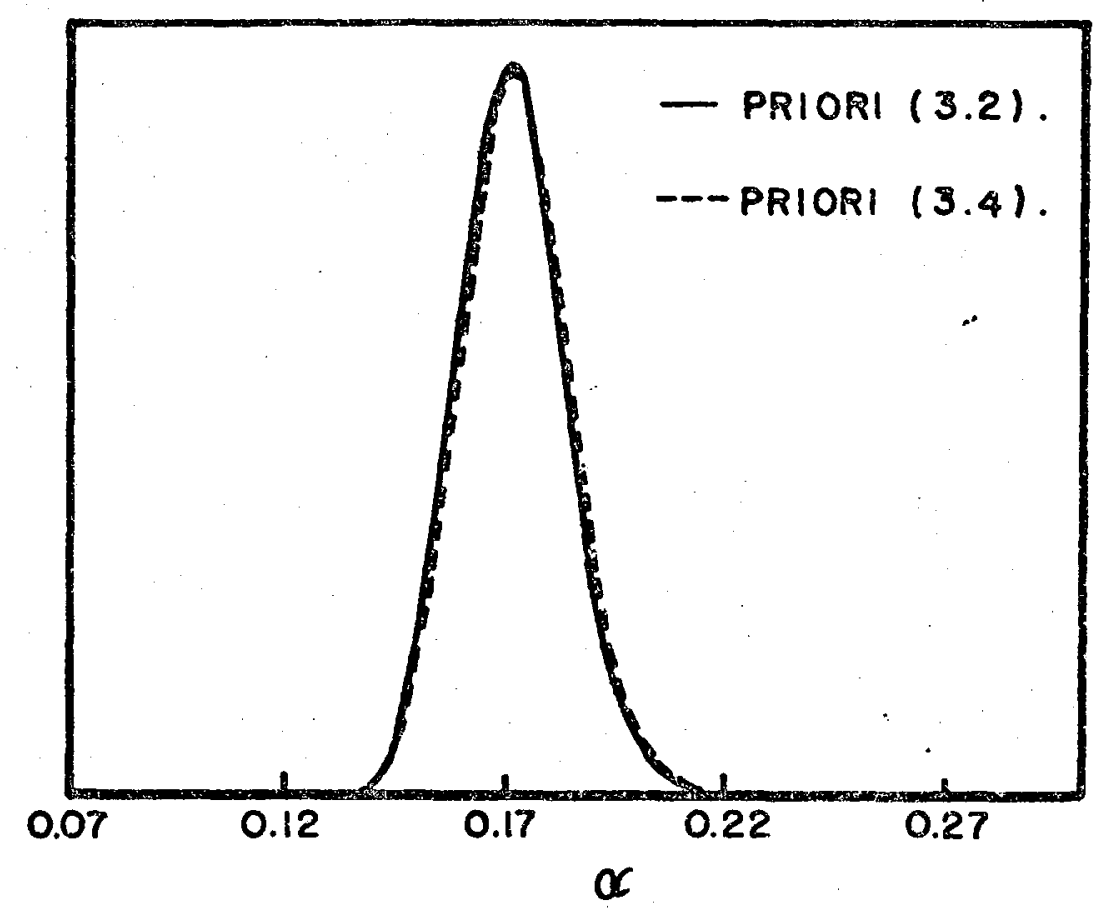

Figura 8.3 Gráfico da densidade a posteriori marginal para $\alpha$.

Similarmente construimos (ver figura 8.4) os gráficos das densidades a posteriori marginais para $\beta(3.10)$ e (3.13), ou seja considerando as priori não informativas (3.2) e (3.4), obtidas pelo método de aproximação de LAPLACE. As modas destas densidades a posteriori marginais são dadas por $\widehat{\beta}^{*} \simeq 131.7786$ e $\widehat{\beta}^{*} \simeq 131.7782$, respectivamente. 
Para calcular a moda de ambos parâmetros, considerando as duas priori nãoinformativas utilizamos métodos numéricos. Empregando programas computacionais simples e de fácil solução em qualquer microcomputador.

Para a construção dos intervalos de credibilidade aproximados para $\alpha$ e $\beta$ utilizamos métodos numéricos, isto é, achando as áreas aproximadas abaixo das curvas nas figuras 8.3 e 8.4, ou seja determinando numericamente os pontos $\alpha_{0}, \alpha_{1}, \beta_{0}$ e $\beta_{1}$ tais que, $P\left(\alpha_{0} \leq \alpha \leq \alpha_{1}\right)=0.90$ e $P\left(\beta_{0} \leq \beta \leq \beta_{1}\right)=0.90$, respectivamente. Desta maneira os intervalos de credibilidade $90 \%$ aproximados para $\alpha$ são dados por $(0.1528 ; 0.1916)$ e $(0.1535 ; 0.1939)$ considerando as priori (3.2) e (3.4), respectivamente, e para $\beta$ são dados por $(128.15 ; 135.56)$ e $(128.15 ; 135.60)$.

Utilizando a aproximação Normal e considerando a priori (3.4) os intervalos de credibilidade para $\alpha$ e $\beta$ são dados por $(0.1506 ; 0.1902)$ e $(128.61 ; 135.39)$, respectivamente.

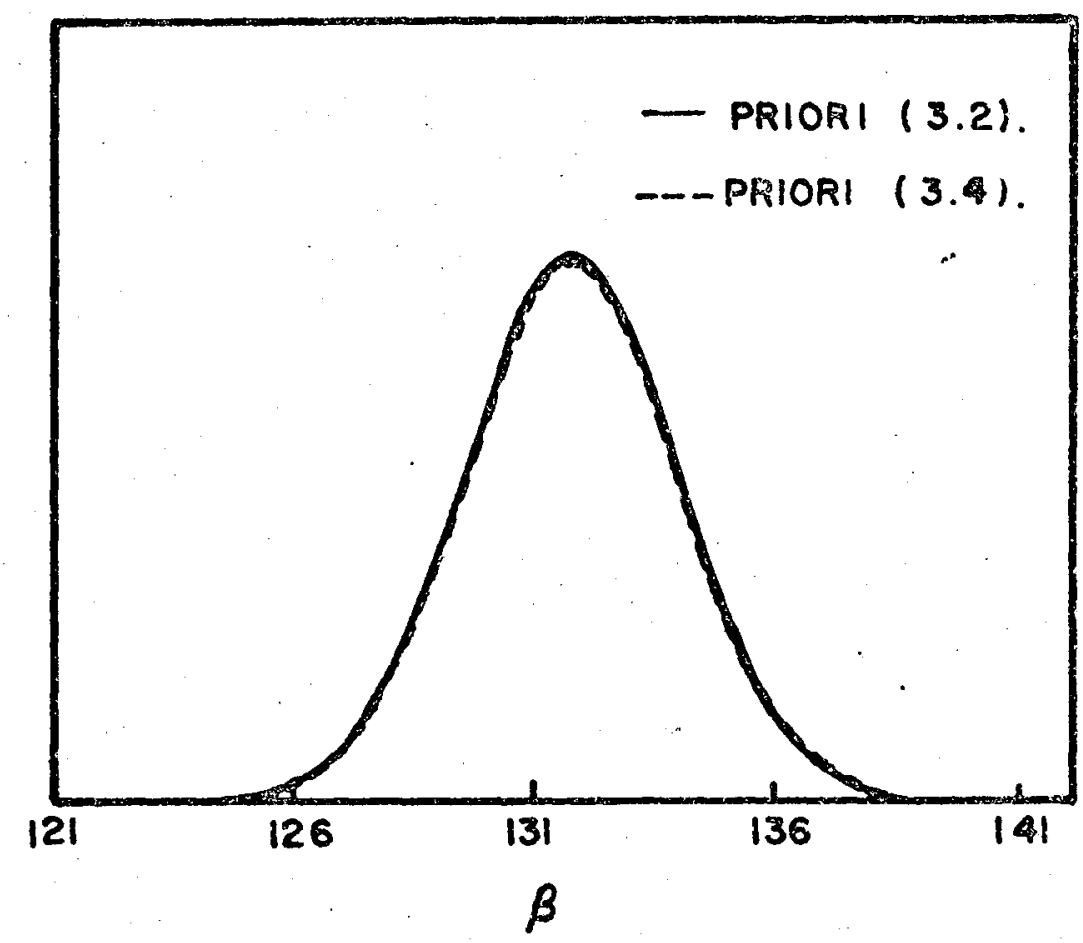

Figura 8.4 Gráfico da densidade a posteriori marginal para $\beta$. 


\subsubsection{Comparação entre as estimativas pontuais e as estimati- vas por intervalo.}

Na tabela 8.2 temos os estimadores de máxima verossimilhança e as modas das densidades a posteriori para os parâmetros $\alpha$ e $\beta$.

Tabela 8.2 Estimadores de máxima verossimilhança e modas a posteriori para os parâmetros $\alpha$ e $\beta$.

\begin{tabular}{c|l|r}
\hline Parâmetro. & $\begin{array}{l}\text { Estimador de máxima } \\
\text { verossimilhança. }\end{array}$ & $\begin{array}{r}\text { Moda a Posteriori } \\
\text { (Aproximada) }\end{array}$ \\
\hline$\alpha$ & $\widehat{\alpha}=0.1704$ & $\widehat{\alpha}^{*} \simeq 0.1696$ (Priori 3.2). \\
& & $\widehat{\alpha}^{*} \simeq 0.1704$ (Priori 3.4) \\
\hline$\beta$ & $\widehat{\beta}=131.8200$ & $\widehat{\beta}^{*} \simeq 131.7786$ (Priori 3.2) \\
& & $\widehat{\beta}^{*} \simeq 131.7782$ (Priori 3.4) \\
\hline
\end{tabular}

Da tabela 8.2 podemos observar que os valores das estimativas pontuais para todos os parâmetros são muito próximos. Portanto, neste exemplo poderíamos utilizar as densidades a posteriori marginais considerando a priori de TIBSHIRANI para simplificar os cálculos. e $\beta$.

$\mathrm{Na}$ tabela 8.3 temos os intervalos de confiança e de credibilidade $90 \%$ para $\alpha$

Tabela 8.3 Intervalos de confiança e de credibilidade $90 \%$ para os parâmetros $\alpha$ e $\beta$.

\begin{tabular}{l|l|l|l}
\hline Tipo de intervalo. & Procedimento & Parâmetro $\alpha$ & Parâmetro $\beta$. \\
\hline $\begin{array}{l}\text { Normalidade assintótica } \\
\text { (Intervalo de confiança) }\end{array}$ & $\begin{array}{l}\text { LAPLACE) } \\
\text { (SIMPSON) }\end{array}$ & $(0.1506 ; 0.1902)$ & $(128.14 ; 135.49)$ \\
\hline $\begin{array}{l}\text { Densidade a posteriori } \\
\text { (intervalo de }\end{array}$ & $\begin{array}{l}\text { Integração } \\
\text { numerica. }\end{array}$ & $(0.1528 ; 0.1916)^{*}$ & $(128.15 ; 135.49)$ \\
\cline { 2 - 4 } $\begin{array}{l}\text { credibilidade, } \\
\text { aproximado). }\end{array}$ & $\begin{array}{l}\text { Aproximação } \\
\text { Normal. }\end{array}$ & $(0.1535 ; 0.1939)^{* *}$ & $(128.15 ; 135.56)^{*}$ \\
\hline
\end{tabular}

NOTA: ${ }^{*}$ representa a priori $(3.2) \mathrm{e}^{* *}$ representa a priori $(3.4)$. 
Da tabela 8.3 podemos concluir que os intervalos obtidos para ambos parâmetros são aproximadamente os mesmos considerando os dois enfoques. Mas pela simplicidade e pela precisão é preferível utilizar as densidades a posteriori marginais (3.8) e (3.13).

\subsubsection{Análise Bayesiana assumindo $\beta$ conhecido $(\beta=132)$.}

Novamente considerando os dados da tabela 8.1 e assumindo $\beta=132$, construimos (ver figura 8.5) o gráfico da densidade a posteriori para $\alpha$ (3.16). A moda desta densidade a posteriori é dada por $\hat{\alpha}^{*} \simeq 0.1696$.

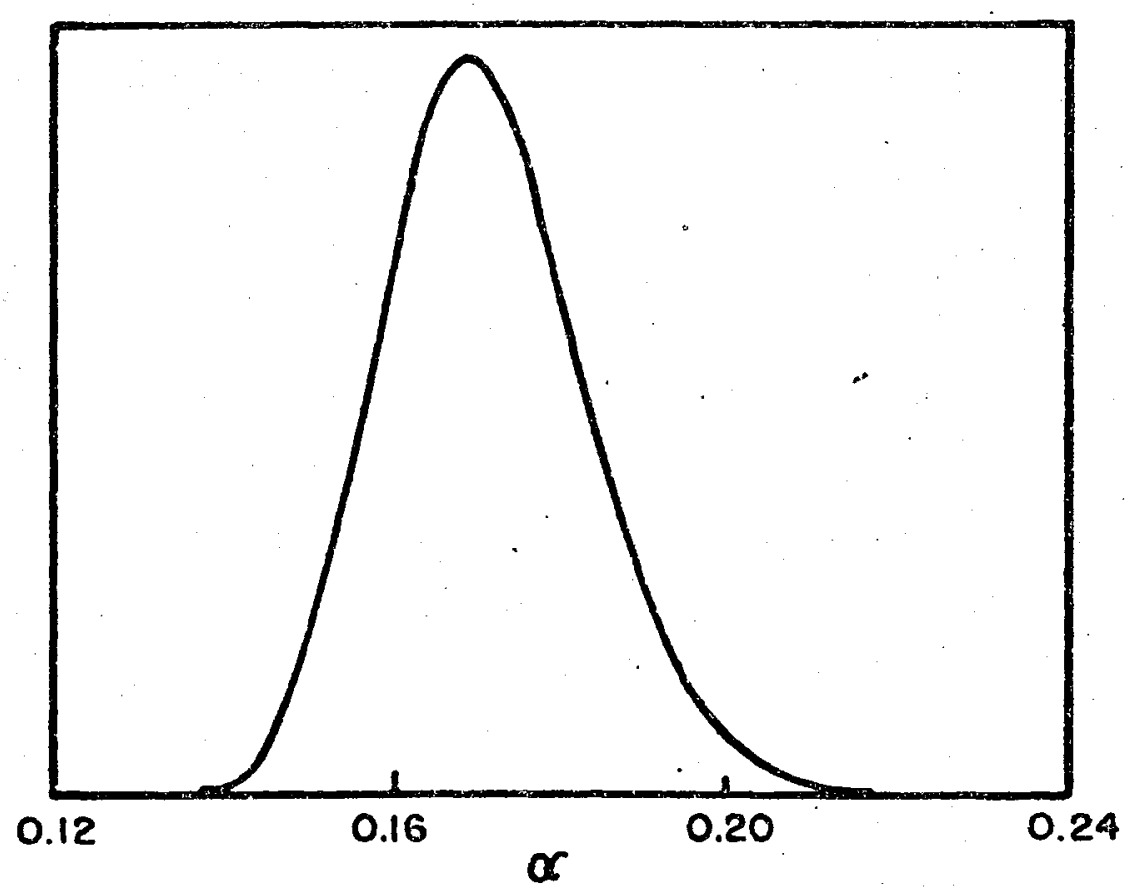

Figura 8.5 Gráfico da densidade a posteriori marginal para $\alpha \operatorname{com} \beta=132$.

Também assumindo $\beta=132$, construimos (ver figura 8.6) o gráfico da densidade a posteriori para o tempo médio de vida da fadiga $\xi$ (3.18). Utilizando a expressão (3.19) a moda desta densidade é dada por $\hat{\xi}^{*}=133.8793$, e utilizando a expressão (3.20) um intervalo de credibilidade para $\xi$ é dado por $(133.55 ; 134.50)$.

Assumindo $\beta=132$ construimos (ver figura 8.7) o gráfico da densidade preditiva para a observação futura $t_{102}$ (3.23). A moda da densidade preditiva é dada por 
$\hat{t}_{102}^{*} \simeq 128.23$. A expressão (3.23) pode ser utilizada para testes de controle de qualidade com a distribuição de Birnbaum-Saunders.

Tabela 8.4 Alguns valores de $P(\alpha /$ dados $)$ considerando o método de LAPLACE e a regra de SIMPSON (Priori (3.4)).

\begin{tabular}{ccc}
\hline \multicolumn{3}{c}{$P(\alpha /$ dados $)$} \\
\hline$\alpha$ & SIMPSON & LAPLACE \\
\hline 0.12 & 0.0000 & 0.0000 \\
0.13 & 0.0044 & 0.0040 \\
0.14 & 0.3793 & 0.3551 \\
0.15 & 5.5000 & 5.2815 \\
0.16 & 21.7127 & 21.2906 \\
0.17 & 32.9546 & 32.8815 \\
0.18 & 24.6058 & 24.9143 \\
0.19 & 10.8118 & 11.0852 \\
0.20 & 3.1907 & 3.3068 \\
0.21 & 0.6980 & 0.7307 \\
0.22 & 0.1220 & 0.1286 \\
0.23 & 0.0180 & 0.0191 \\
0.24 & 0.0024 & 0.0025 \\
0.25 & 0.0003 & 0.0003 \\
0.26 & 0.0000 & 0.0000 \\
\hline
\end{tabular}

NOTA: regra de SIMPSON (ver por exemplo, Zellner, 1971).

$\mathrm{Na}$ tabela 8.4 temos alguns valores de $P(\alpha /$ dados $)$ considerando o método de LAPLACE e a regra de SIMPSON assumindo a priori (3.4). Observamos boa precisão para a densidade a posteriori aproximada pelo método de LAPLACE. 


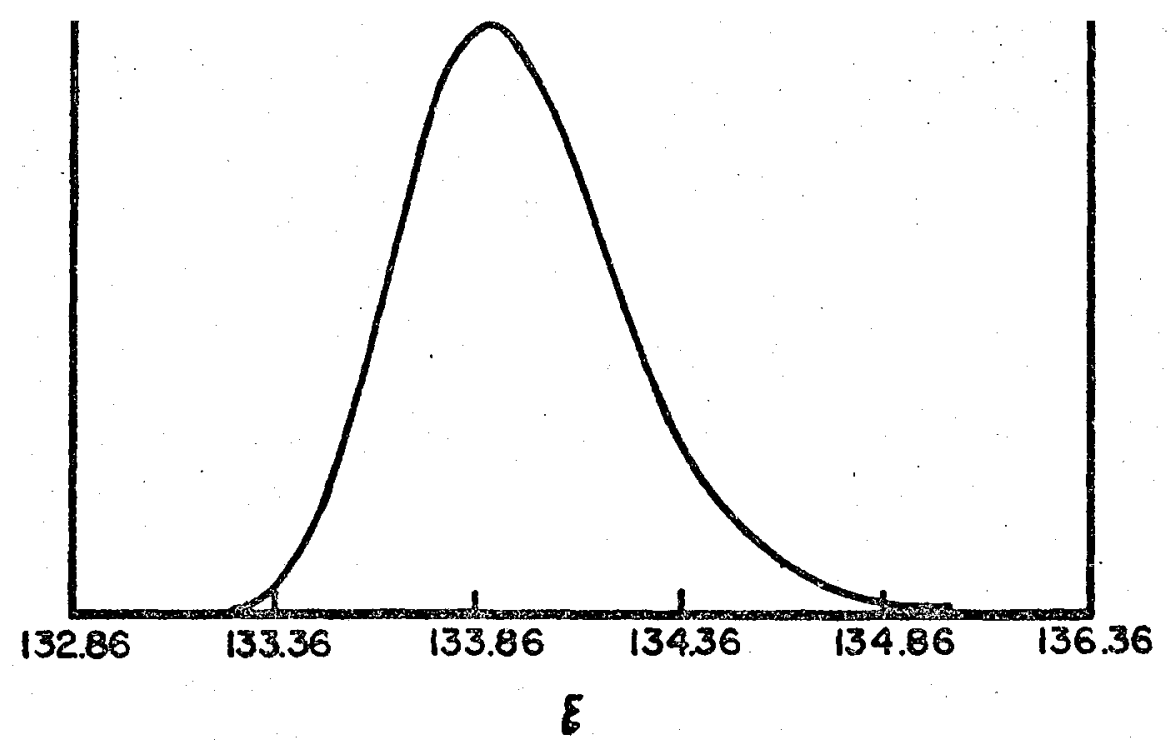

Figura 8.6 Gráfico da densidade a posteriori para $\xi \operatorname{com} \beta=132$.

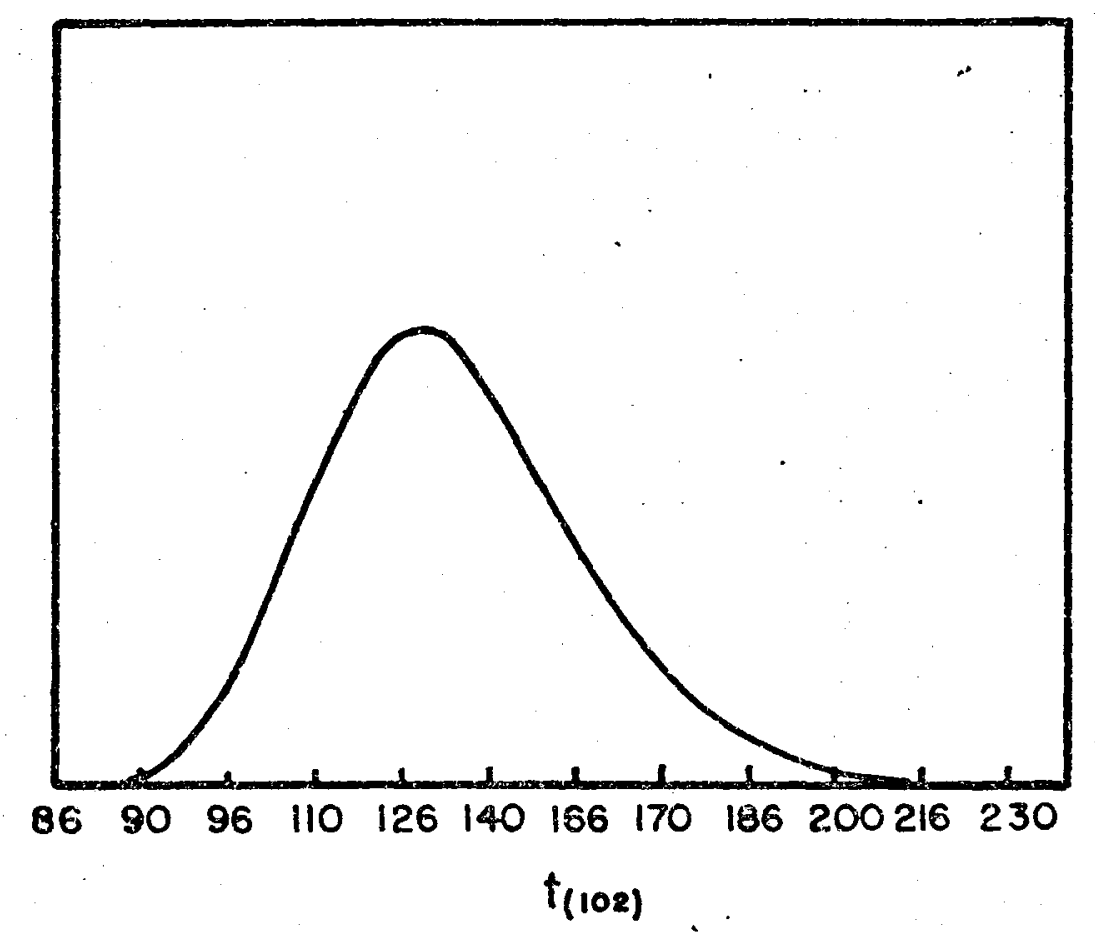

Figura 8.7 Gráfico da densidade preditiva para $t_{(102)} \operatorname{com} \beta=132$. a e b. 


\subsection{Exemplo 2}

\subsubsection{Descrição dos dados.}

$\mathrm{Na}$ tábela 8.5, apresentamos um conjunto de dados utilizados por Brown e Miller (1978). Os valores de $W_{c}$ (tensão por ciclo) foram calculados por Bhargava (1984). Estes dados representam resultados de teste de fadiga em aço com composição de $1 \%$ de $C_{r}-M_{0}-V$, onde corpos de prova em forma cilíndrica foram submetidos a torções $\mathrm{e}$ cargas axial combinadas sob uma amplitude constante de ciclos até sua falha.

A partir de considerações empíricas, consideramos o modelo $\ln (N)=a+b x$, onde $x=\ln \left(W_{c}\right)$, (ver (1.17)), e $W_{c}$ é a tensão por ciclo; $\mathrm{N}$ é o número de ciclos até a falha de cada corpo de prova. Este conjunto de dados também foi considerado e analizado por Rieck e Nedelman, 1991.

Tabela 8.5 Dados de fadiga com dois eixos.

\begin{tabular}{rrrrrrrr}
\hline$W_{c}$ & $\mathrm{~N}$ & $W_{c}$ & $\mathrm{~N}$ & $W_{c}$ & $\mathrm{~N}$ & $W_{c}$ & $\mathrm{~N}$ \\
\hline 11.5 & 3280 & 24.0 & 804 & 40.1 & 750 & 60.3 & 283 \\
13.0 & 5046 & 24.6 & 1093 & 40.1 & 316 & 60.5 & 212 \\
14.3 & 1563 & 25.2 & 1125 & 43.0 & 456 & 62.1 & 327 \\
15.6 & 4707 & 25.5 & 884 & 44.1 & 552 & 62.8 & 373 \\
16.0 & 977 & 26.3 & 1300 & 46.5 & 355 & 66.5 & 125 \\
17.3 & 2834 & 27.9 & 852 & 47.3 & 242 & 67.0 & 187 \\
19.3 & 2266 & 28.3 & 580 & 48.7 & 190 & 67.1 & 135 \\
21.1 & 2208 & 28.4 & 1066 & 52.9 & 127 & 67.9 & 245 \\
21.5 & 1040 & 28.6 & 1114 & 56.6 & 185 & 68.8 & 137 \\
22.6 & 700 & 30.9 & 386 & 59.9 & 255 & 75.4 & 200 \\
22.6 & 1583 & 31.9 & 745 & 60.2 & 195 & 100.5 & 190 \\
24.0 & 482 & 34.5 & 736 & & & & \\
\hline
\end{tabular}

\subsubsection{Verificação gráfica da adequabilidade do modelo.}

Para verificar a adequabilidade do modelo Log-Linear da distribuição de Birnbaum-Saunders utilizaremos um procedimento gráfico. Considerando a expressão (1.17) que relaciona o logaritmo do número de ciclos para a amostra falhar com o logaritmo da 
tensão por ciclo (ver; figura 8.8), ou seja construindo o gráfico de $\ln (N)$ versus $\ln \left(W_{c}\right)$, podemos verificar a existência ou nẫo de uma rclação linear aproximada. Caso exista, dizemos que o modelo Log-Linear para a distribuição de Birnbaum-Saunders (4.4) é válido para a amostra em estudo.

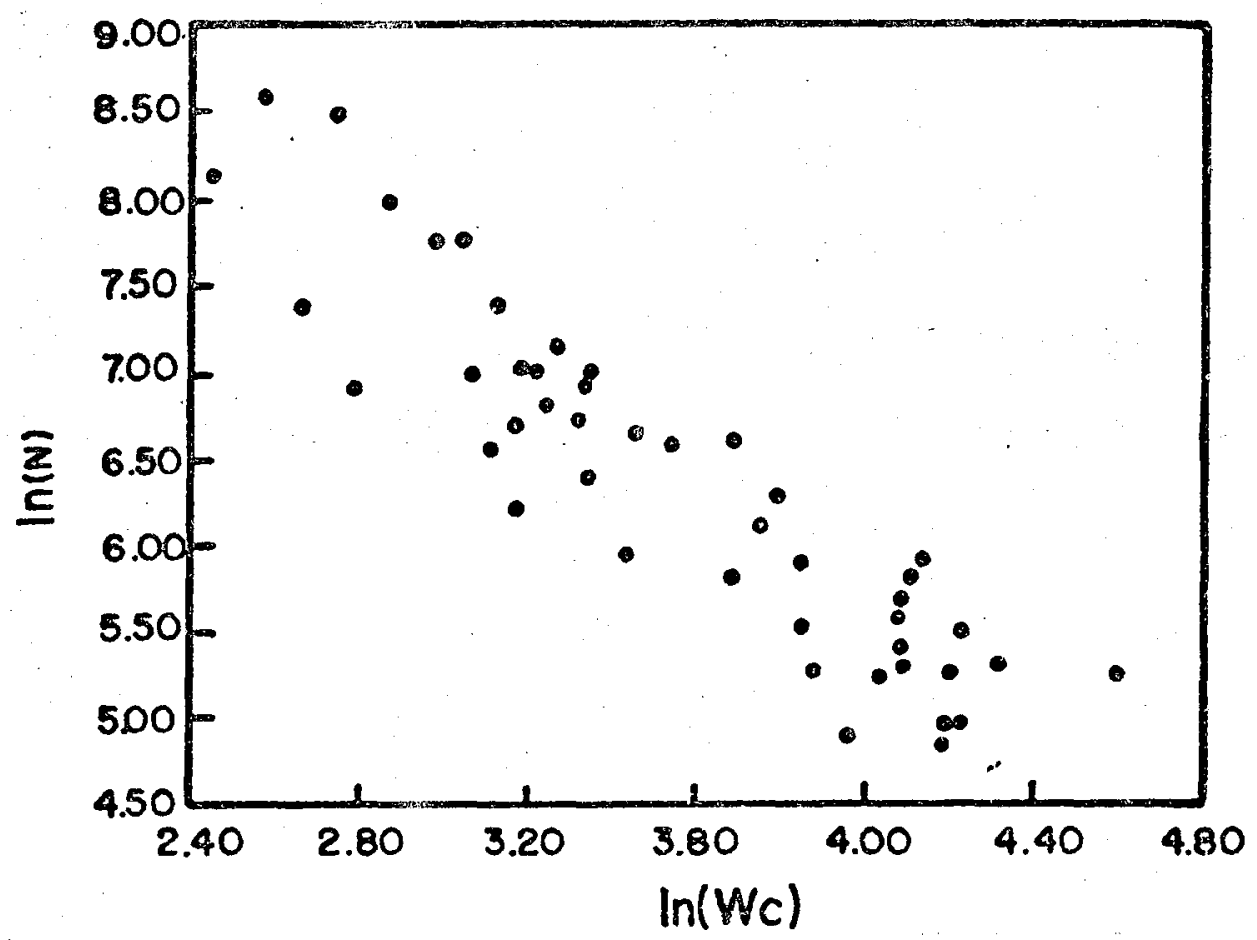

Figura 8.8 Gráfico de $\ln (N)$ versus $\ln \left(W_{c}\right)$.

Da figura 8.8, podemos concluir que existe uma relação linear aproximada. Isto implica que o modelo Log-Linear da distribuição de Birnbaum-Saunders com uma variável Stress (4.4) é adequado para o conjunto de dados da tabela 8.5.

\subsubsection{Análise clássica do problema.}

Nesta seção, utilizando a metodología obtida no capítulo 4 efetuaremos uma análise clássica (para os dados da tabela 8.5), baseada na aproximação normal assintótica dos estimadores de máxima verossimilhança dos parânietros envolvidos. Os estimadores de máxima verossimilhança para $\mathrm{a}, \mathrm{b}$ e $\alpha$ obțidos numericamente são dados por $\widehat{a}=$ $12.28(0.3890), \widehat{b}=-1.671(0.1083)$ e $\hat{\alpha}=0.41(0.0427)$. Os valores entre parenteses são os respectivos desvios padrões estimados dos parâmetros considerando a matriz de informação observada (4.13). 
Considerando a aproximação normal assintótica dos estimadores de máxima verossimilhança $\widehat{a}, \hat{b}$ e $\hat{\alpha}$ dada em (4.12) podemos construir intervalos de confiança 90 $\%$ para a,b e $\alpha$. Assim utilizando a matriz de informação observada, os intervalos de confiança $90 \%$ para a, b e $\alpha$ são $(11.52 ; 13.04),(-1.88 ;-1.48)$ e $(0.33 ; 0.49)$ respectivamente. Considerando a matriz de informação de Fisher em sua forma simplificada (4.33) os intervalos de confiança $90 \%$ são dados por $(11.63 ; 12.93),(-1.85 ;-1.49)$ e $(0.34 ; 0.48)$.

\subsubsection{Análise Bayesiana do problema, assumindo $\mathrm{a}$, $\mathrm{b}$ e $\alpha$ des- conhecidos.}

Nos capítulos 3 e 5 vimos que em inferência Bayesiana, as inferências sobre os parâmetros de interesse podem ser realizadas utilizando a suas densidades a posteriori marginais. Portanto considerando os dados da tabela 8.5, construimos (ver figura 8.9) os gráficos das densidades a posteriori marginais para $\alpha(5.7)$ e (5.9), obtidas pelo método de aproximação de LAPLACE. As modas destas densidades a posteriori aproximadas são dadas por 0.406 e 0.415 , respectivamente. Observe que os resultados são muito próximos, isto é, na prática podemos utilizar a forma simplificada da marginal (5.9).

Para calcular a moda de ambos parâmetros, considerando as duas prioris nãoinformativas utilizamos métodos numéricos, empregando programas computacionais de solução simples em qualqựr microcomputador.

Para a construaão dos intervalos de credibilidade aproximados para $\alpha$, utilizamos métodos numéricos achando as áreas aproximadas abaixo das curvas na figura 8.9, ou seja determinando num rricamente os pontos $\alpha_{0}, \alpha_{1}$, tais que, $P\left(\alpha_{0} \leq \alpha \leq \alpha_{1}\right)=0.90$. Desta maneira os interval ss de credibilidade $90 \%$ aproximados para $\alpha$ são dados por $(0.352 ; 0.498)$ e $(0.358 ; 0.51 \phi)$, respectivamente.

Na figura 8.10, fpresentamos o gráfico dos contornos da densidade a posteriori marginal conjunta para a e b dada na expressão (5.12). A moda desta densidade a posteriori marginal conjun a aproximada é dada por $\widehat{a}^{*} \simeq 12.28 \mathrm{e} \widehat{b}^{*} \simeq-1.671$. Intervalos de credibilidade $90 \%$ aprołimados para a e b, são dados por $(11.65 ; 12.91)$ e $(-1.85 ;-1.50)$, respectivamente.

Do gráfico dos fontornos, podemos observar uma boa forma elíptica, isto é, boa normalidade para a densidade a posteriori conjunta de a e b, com uma correlação negativa. 


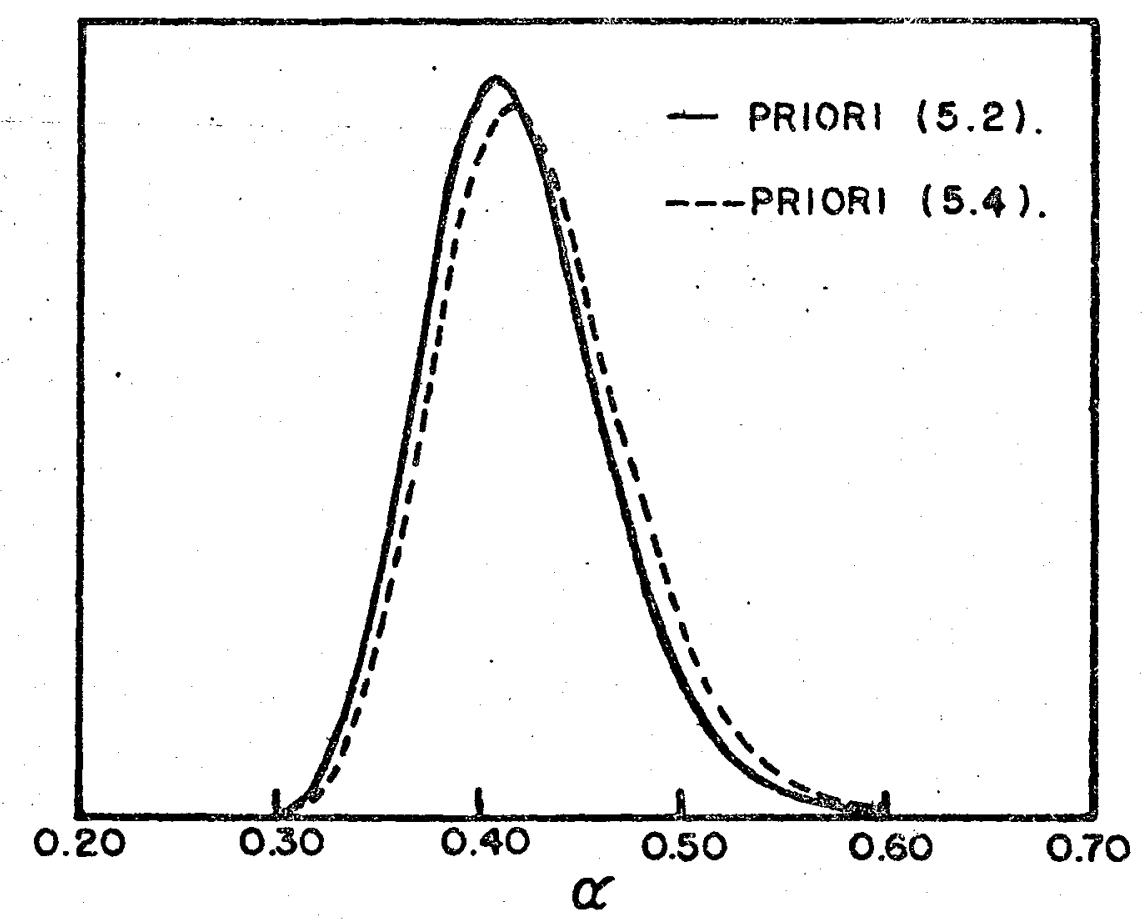

Figura 8.9 Gráfico da densidade a posteriori marginal para $\alpha$.

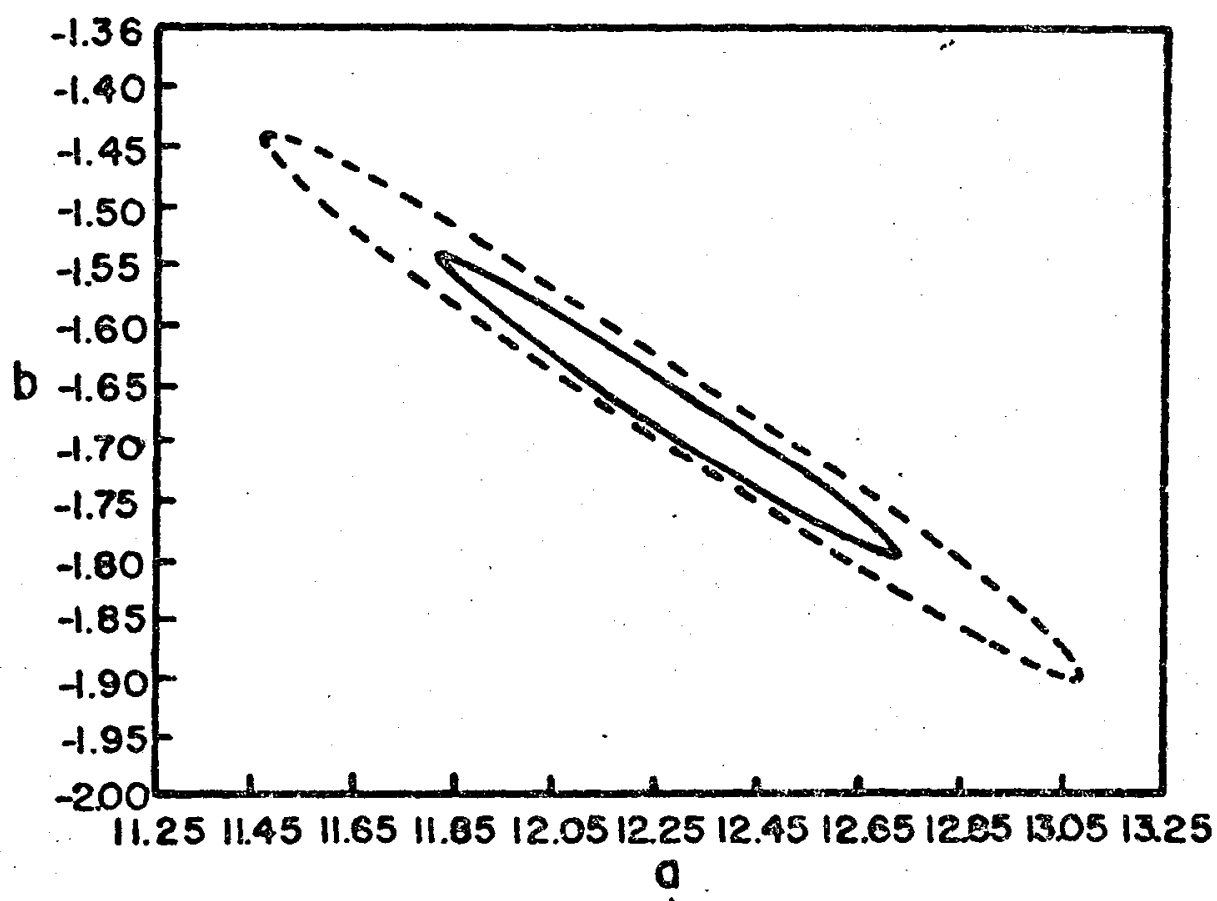

Figura 8.10 Gráfico dos contornos para a densidade a posteriori conjunto de a c b. 


\subsubsection{Comparação entre as estimativas pontuais e as estimati- vas por intervalo.}

Na tabela 8.6 temos os estimadores de máxima verossimilhança e as modas das densidades a posteriori para os parâmetros $a, b$ e $\alpha$.

Tabela 8.6 Estimadores de máxima verossimilhança e modas a posteriori para os parâmetros $a, b$ e $\alpha$.

\begin{tabular}{c|l|r}
\hline Parâmetro. & $\begin{array}{l}\text { Estimador de máxima } \\
\text { verossimilhança. }\end{array}$ & $\begin{array}{r}\text { Moda a Posteriori } \\
\text { (Aproximada). }\end{array}$ \\
\hline$a$ & $\widehat{a}=12.28$ & $\widehat{a}^{*} \simeq 12.28$ (Priori 5.2). \\
\hline$b$ & $\widehat{b}=-1.671$ & $\widehat{b}^{*} \simeq-1.671$ (Priori 5.2). \\
\hline$\alpha$ & $\widehat{\alpha}=0.41$ & $\widehat{\alpha}^{*} \simeq 0.406$ (Priori 5.2). \\
& & $\hat{\alpha}^{*} \simeq 0.415$ (Priori 5.4). \\
\hline
\end{tabular}

Da tabela 8.6 podemos observar que os valores das estimativas pontuais para todos os parâmetros são muito próximos. Portanto neste exeníplo, poderíamos utilizar as densidades a posteriori marginais para simplificar os cálculos. $b$ e $\alpha$.

$\mathrm{Na}$ tabela 8.7 temos os intervalos de confiança e de credibilidade $90 \%$ para $a$,

Tabela 8.7 Intervalos de confiança $e$ de credibilidade $90 \%$ para os parâmetros $a, b$ e $\alpha$.

\begin{tabular}{l|l|l|l}
\hline Tipo de intervalo. & Parâmetro $a$ & Parâmetro $b$ & Parâmetro $\alpha$. \\
\hline $\begin{array}{l}\text { Normalidade assintótica } \\
\text { (Intervalo de confiança) }\end{array}$ & $(11.52 ; 13.04) \mathrm{O}$ & $(-1.88 ;-1.48) \mathrm{O}$ & $(0.33 ; 0.49) \mathrm{O}$ \\
\hline $\begin{array}{l}\text { Densidade a posteriori } \\
\text { (intervalo de credibilidade } \\
\text { aproximado). }\end{array}$ & $(11.63 ; 12.93) \mathrm{R}$ & $(-1.85 ;-1.49) \mathrm{R}$ & $(0.34 ; 0.48) \mathrm{R}$ \\
\hline
\end{tabular}

NOTA (a): * representa a priori (5.2) $\mathrm{e}^{* *}$ representa a priori (5.4).

NOTA (b): O representa a matriz (4.13) e $\mathrm{R}$ representa a matriz (4.33). 


\subsubsection{Análise Bayesiana assumindo $\alpha$ conhecido $(\alpha=0.40)$.}

Considerando os dados da tabela 8.5 e assumindo $\alpha=0.40$, construimos (ver figura 8.11) o gráfico da densidade a posteriori marginal aproximada para a (5.19), obtido por integração numérica e pela aproximação de LAPLACE. Um intervalo de credibilidade aproximado a $90 \%$ para a considerando $\alpha=0.40$, é dado por $(11.66 ; 12.91)$. A moda desta densidade a posteriori marginal para a é dada por $\widehat{a}^{*} \simeq 12.28$.

Também assumindo $\alpha=0.40$, construimos (ver figura 8.12) o gráfico da densidade a posteriori marginal aproximada para b (5.21), obtido por integração numérica e pela aproximação de LAPLACE. Um intervalo de credibilidade aproximado a $90 \%$ para $\mathrm{b}$ considerando $\alpha=0.40$, é dado por $(-1.85 ;-1.50)$. A moda desta densidade a posteriori marginal para $\mathrm{b}$ é dada por $\widehat{b}^{*} \simeq-1.671$. Observe a boa aproximação de LAPLACE (ver tabela 8.8).

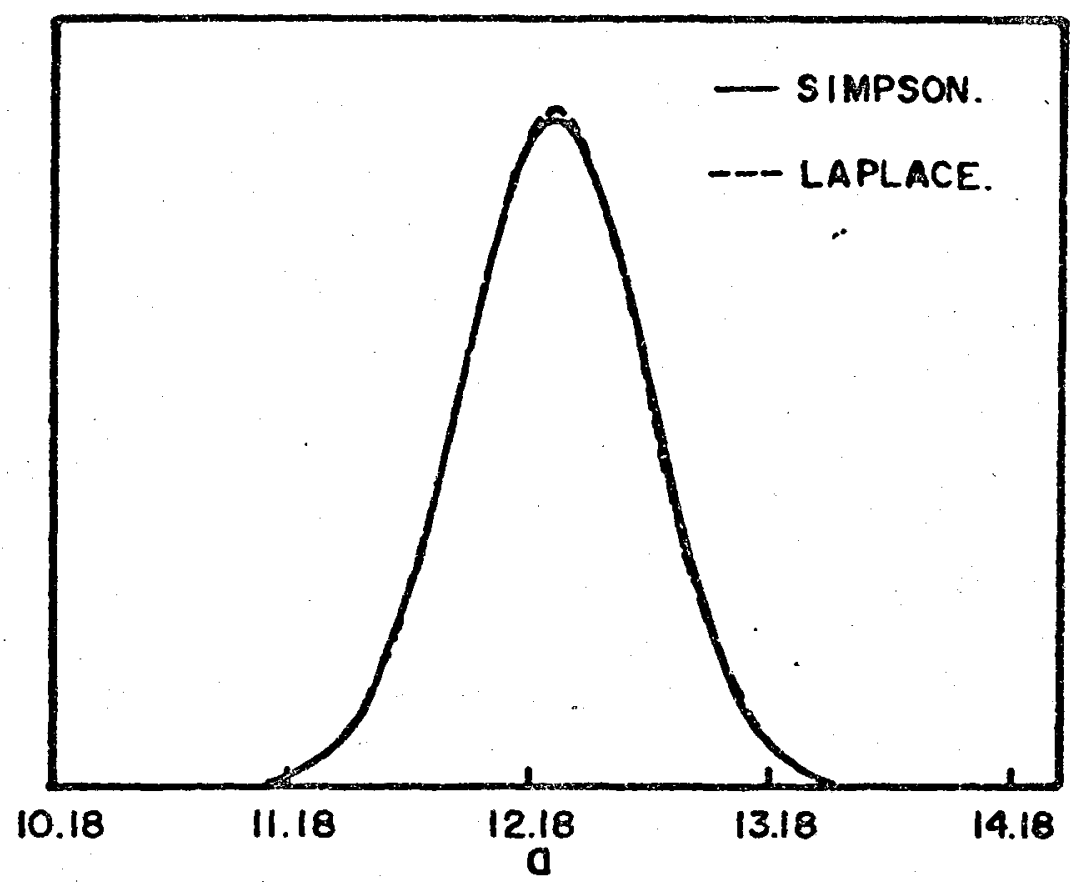

Figura 8.11 Gráfico da densidade a posteriori marginal para a com $\alpha=0.40$. 


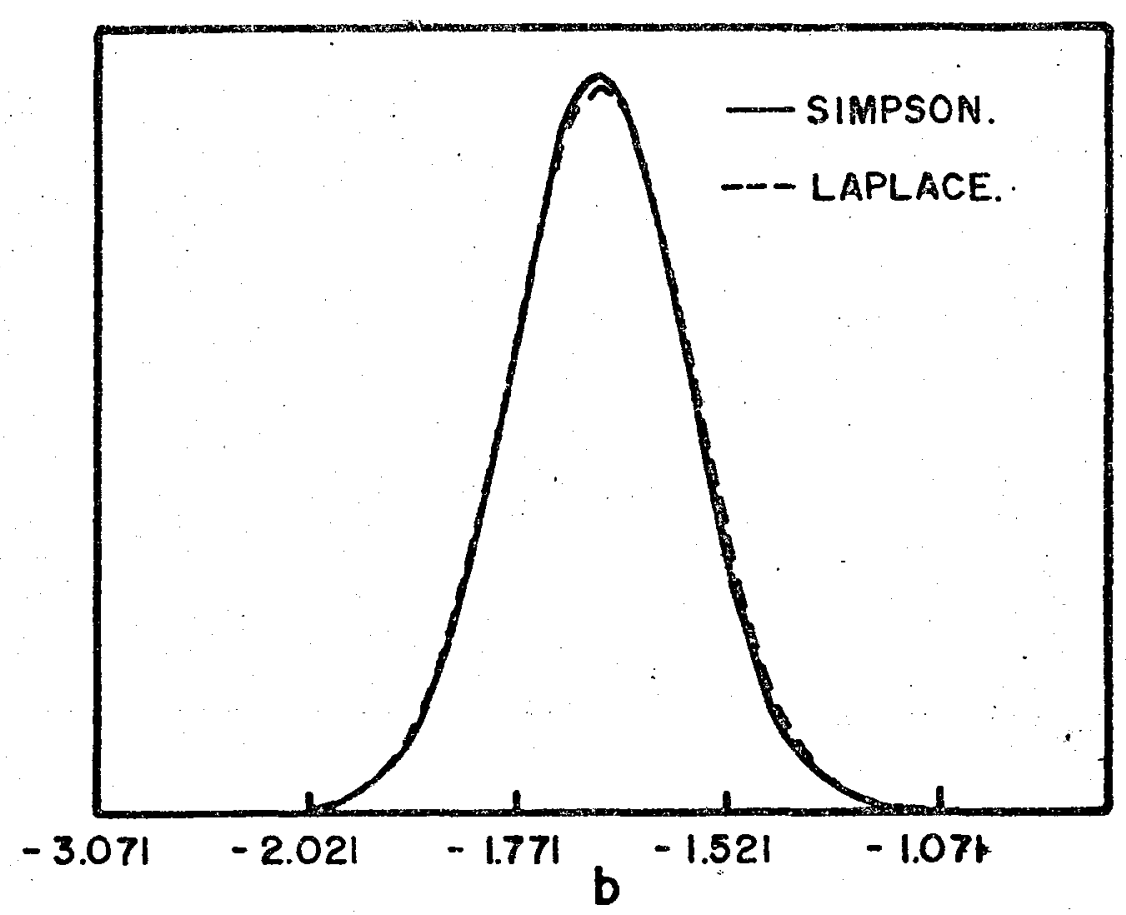

Figura 8.12 Gráfico da densidade a posteriori para $b$ com $\alpha=0.40$.

De igual forma assumindo $\alpha=0.40$, construimos (ver figura 8.13) o gráfico da densidade a posteriori para o tempo médio de vida $\lambda(5.24)$, considerando $W_{c}=20$, $W_{c}=40$ e $W_{c}=120$. As modas aproximadas para o tempo médio de vida $\lambda$, dos valores $W_{c}$ acima considerados são dadas por $7.27,6.12$ e 4.28 , e os intervalos de credibilidade a $90 \%$ aproximados são dados por $(7.14 ; 7.40),(6.00 ; 6.19)$ e $(4.00 ; 4.47)$, respectivamente.

Finalmente, também assumindo $\alpha=0.40$ construimos (ver figura 8.14) o gráfico da densidade preditiva para a observação futura $y_{(n+1)}=\ln (N)$, considerando $W_{c}=20, W_{c}=40 \mathrm{e} W_{c}=120$. As modas aproximadas para a observação futura $y_{(n+1)}=\ln (N)$, dos valores $W_{c}$ acima considerados são dadas por $7.27,6.16$ e 4.38, e os intervalos de credibilidade a $90 \%$ aproximados são dados por $(7.20 ; 7.35),(6.09 ; 6.21)$ e $(4.19 ; 4.48)$, respectivamente. Desta maneira, a expressão $(5.28)$ pode ser utilizada para realizar previsões e formular testes de controle de qualidade na área de engenharia de materiais.

É importante salientar que é necessário ter muito cuidado em se fazer previsões utilizando os valores da variável $W_{c}$ muito adiante da situação experimental, pois podemos ter boa aproximação, somente dentro da região experimental. 


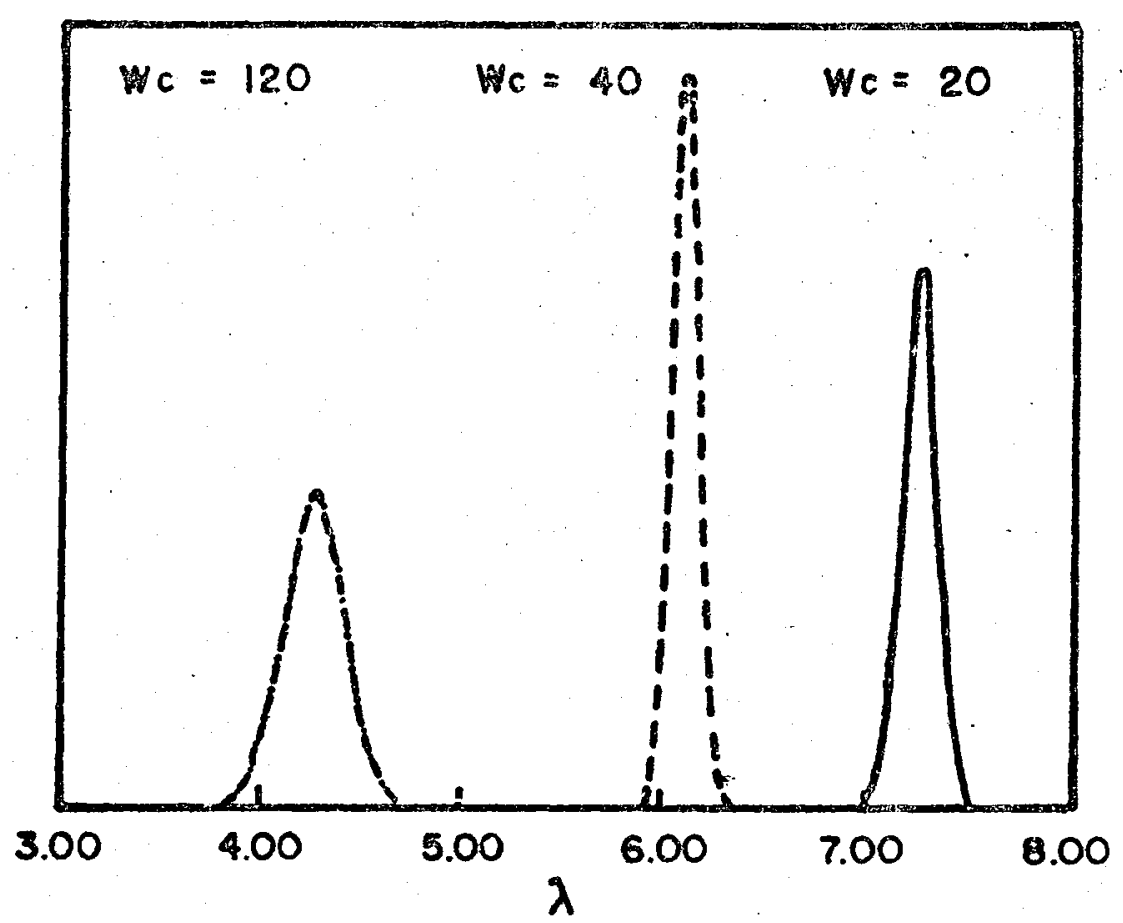

Figura 8.13 Gráfico da densidade a posteriori märinal para o tempo medio de vida $\lambda$, considerando $W_{c}=20, W_{c}=40$ e $W_{c}=120$, com $\alpha=0.40$.

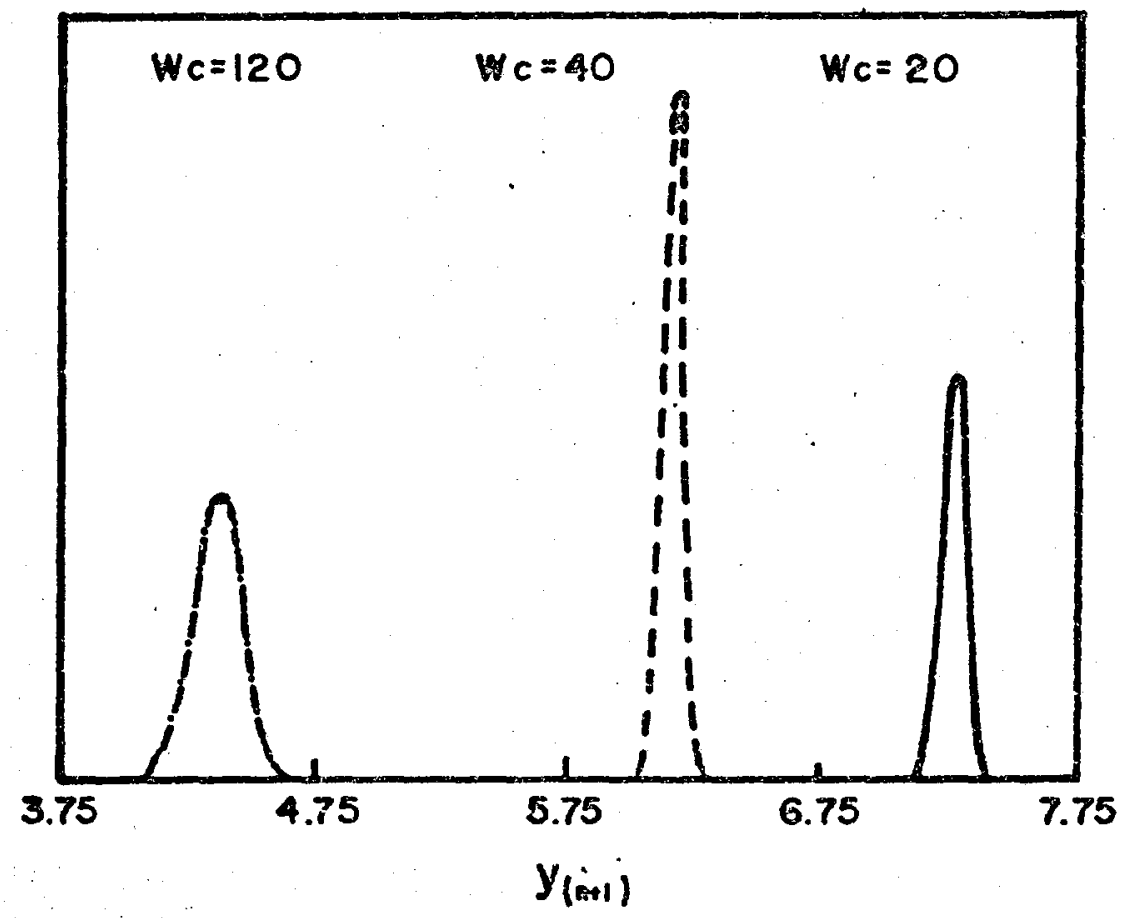

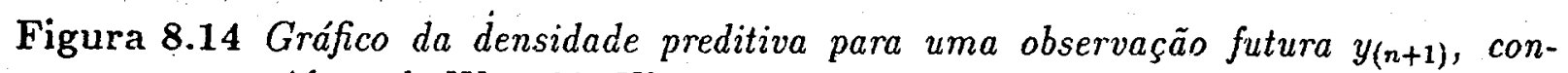
siderando $W_{c}=20, W_{c}=40$ e $W_{c}=120$, com $\alpha=0.40$. 
Tabela 8.8 Alguns valores de $P(a /$ dados $)$ e $P(b /$ dados $)$ considerando o método de $L A$ PLACE e a regra de SIMPSON (para $\alpha=0.40$ ).

\begin{tabular}{ccc|ccc}
\hline & P(a/dados) & \multicolumn{3}{c}{ P(b/dados) } \\
\hline $\mathrm{a}$ & SIMPSON & LAPLACE & $\mathrm{b}$ & SIMPSON & LAPLACE \\
\hline 10.48 & 0.0000 & 0.0000 & -2.191 & 0.0000 & 0.0000 \\
10.68 & 0.0001 & 0.0001 & -2.151 & 0.0001 & 0.0001 \\
10.88 & 0.0011 & 0.0011 & -2.091 & 0.0012 & 0.0016 \\
11.08 & 0.0069 & 0.0070 & -2.031 & 0.0104 & 0.0125 \\
11.28 & 0.0322 & 0.0316 & -1.971 & 0.0639 & 0.0715 \\
11.48 & 0.1134 & 0.1154 & -1.911 & 0.2791 & 0.2960 \\
11.68 & 0.3012 & 0.2941 & -1.851 & 0.8750 & .0 .8921 \\
11.88 & 0.6044 & 0.6148 & -1.791 & 1.9743 & 1.9610 \\
12.08 & 0.9174 & 0.8928 & -1.731 & 3.2148 & 3.1523 \\
12.28 & 1.0538 & 1.0720 & -1.671 & 3.7840 & 3.7115 \\
12.48 & 0.9161 & 0.8899 & -1.611 & 3.2234 & 3.2039 \\
12.68 & 0.6024 & 0.6128 & -1.551 & 1.9878 & 2.0284 \\
12.88 & 0.2993 & 0.2905 & -1.491 & 0.8871 & 0.9416 \\
13.08 & 0.1121 & 0.1141 & -1.431 & 0.2862 & 0.3201 \\
13.28 & 0.0316 & 0.0307 & -1.371 & 0.0667 & 0.0796 \\
13.48 & 0.0067 & 0.0068 & -1.311 & 0.0112 & 0.0144 \\
13.68 & 0.0011 & 0.0010 & -1.251 & 0.0013 & 0.0019 \\
13.88 & 0.0001 & 0.0001 & -1.191 & 0.0001 & 0.0002 \\
14.08 & 0.0000 & 0.0000 & -1.131 & 0.0000 & 0.0000 \\
\hline
\end{tabular}

NOTA: regra de SIMPSON (ver por exemplo, Zellner, 1971).

$\mathrm{Na}$ tabela 8.8 temos alguns valores de $P(a /$ dados $)$ e de $P(b /$ dados $)$ considerando o método de LAPLACE e a regra de SIMPSON (para $\alpha=0.40$ ). Observamos boa precisão para a densidade a posteriori aproximada pelo método de LAPLACE. 


\subsubsection{Análise Bayesiana considerando densidades a priori in- formativas e assumindo $\alpha$ conhecido $(\alpha=0.40)$.}

Na prática usualmente os engenheiros tem opinião a priori sob os parâmetros do modelo. Isto implica que quanto maior a precisão a priori mais fechada será a distribuição a posteriori dos parâmetros, e também em melhores inferências, que podem ser obtidas pelos métodos bayesianos. Como mostramos a seguir, considerando os dados da tabela 8.5 e assumindo $\alpha=0.40$ (ver figuras 8.15 e 8.16 , nas quais PI representa uma densidade a priori informativa e PNI representa uma densidade a priori não informativa).

Na figura 8.15 temos o gráfico da densidade a posteriori marginal aproximada para a (5.19) e (6.13). Para a densidade (6.13), consideramos diferentes valores para $a_{1}, b_{1}, \sigma_{1}, \sigma_{2}$ e $\rho$, a densidade (5.19) será representada com uma linha tracejada e a densidade (6.13) será representada com uma linha continua nos tres casos considerados. Similarmente na figura 8.16 temos o gráfico da densidade a posteriori marginal aproximada para $\mathrm{b}(5.21)$ e (6.15). Para a densidade (6.15), também consideramos diferentes valores para $a_{1}, b_{1}, \sigma_{1}, \sigma_{2}$ e $\rho$, igualmente, representaremos a densidade (5.21) com uma linha tracejada e a densidade (6.15) com uma linha continua.

Os valores considerados para $a_{1}, b_{1}, \sigma_{1}, \sigma_{2}$ e $\rho$, utilizando as densidades (6.13) e (6.15) são dados na tabela 8.9 , em ambos casos representaremos estas densidades por $P_{1}, P_{2}$ e $P_{3}$.

Tabela 8.9 Valores considerados para $a_{1}, b_{1}, \sigma_{1}, \sigma_{2}$ e $\rho$.

\begin{tabular}{c|c|c|c|c|c}
\hline Posteriori & $a_{1}$ & $b_{1}$ & $\sigma_{1}$ & $\sigma_{2}$ & $\rho$ \\
\hline$P_{1}$ & 12.00 & -1.600 & 0.40 & 0.10 & -0.860 \\
\hline$P_{2}$ & 12.28 & -1.671 & 0.40 & 0.10 & -0.988 \\
\hline$P_{3}$ & 12.50 & -1.750 & 0.40 & 0.10 & -0.960 \\
\hline
\end{tabular}

Estudos semelhantes podem ser realizados para as densidades (6.6) e (6.19). 


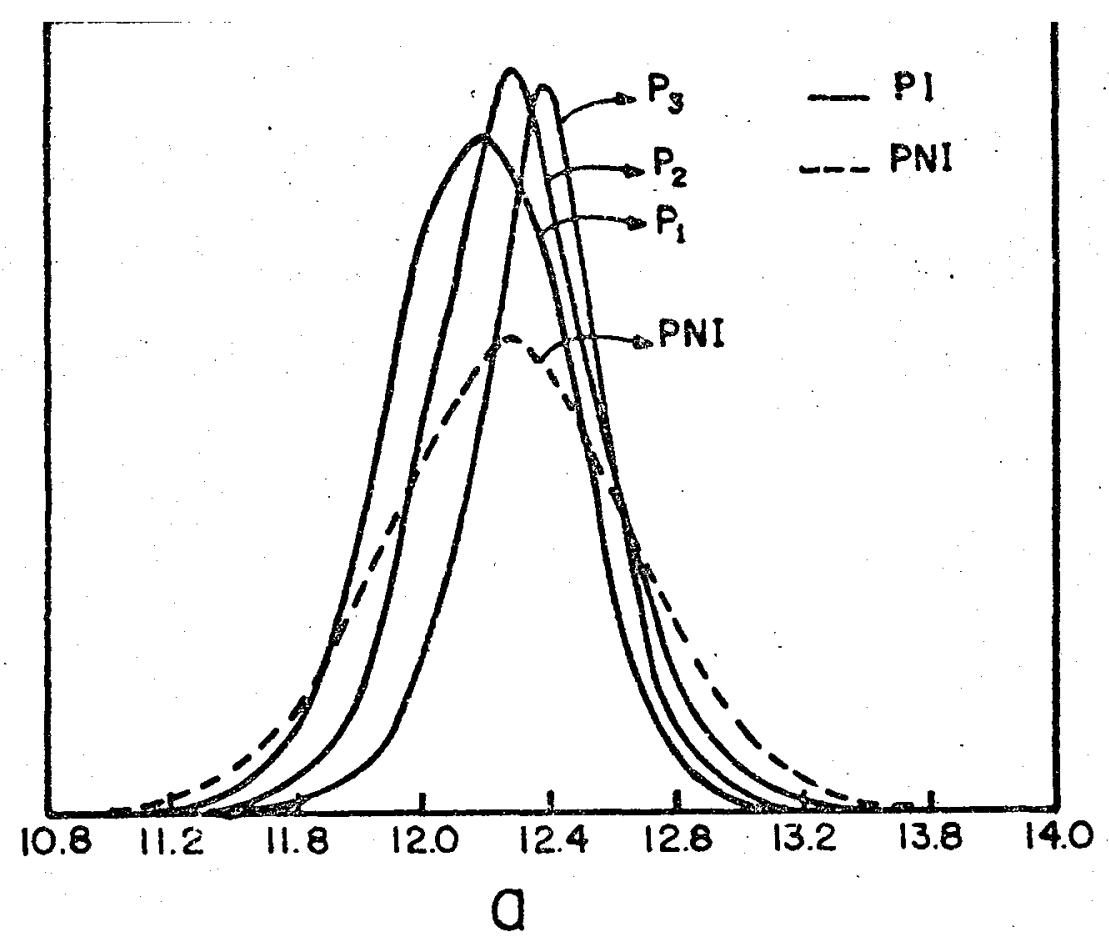

Figura 8.15 Gráfico da densidade a posteriori marginal aproximada para a (5.19) e (6.13).

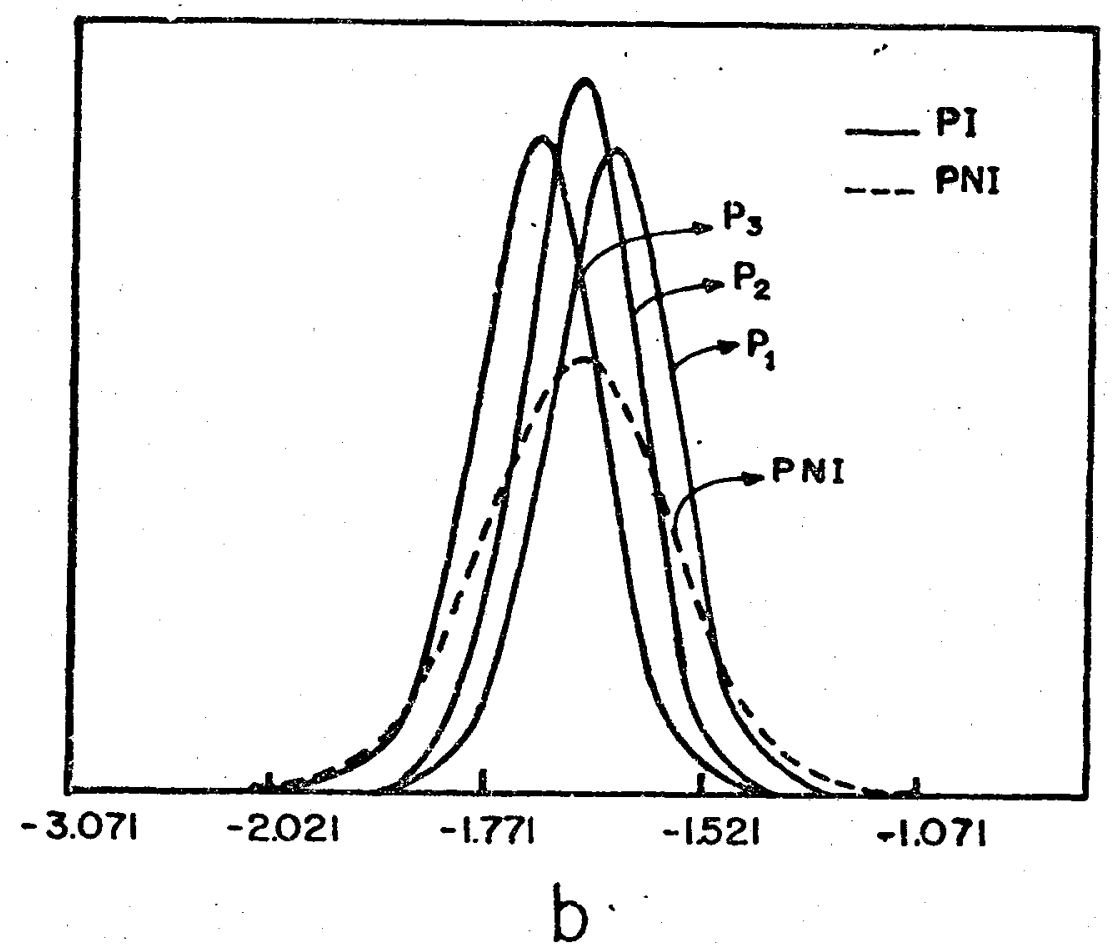

Figura 8.16 Gráfico da densidade a posterirori marginal aproximada para $b$ (5.21) $e$ (6.15). 


\subsection{Exemplo 3}

\subsubsection{Descrição dos dados.}

$\mathrm{Na}$ tabela 8.10 , temos tempos de falha (horas) de dois tipos de materiais isolantes (I e II), testados sob duas temperaturas diferentes. As temperaturas utilizadas foram 200 e 250 graus centígrados. Este conjunto de dados encontra-se no livro de Nelson, 1990.

Tabela 8.10 Tempos de falhas (horas) de dois materiais isolantes.

\begin{tabular}{|c|c|c|c|}
\hline & & $\frac{\text { Mater }}{\mathrm{I}}$ & II \\
\hline \multirow{10}{*}{ Temperatura. } & \multirow{5}{*}{200} & 1176 & 2520 \\
\hline & & 1512 & 2856 \\
\hline & & 1512 & 3192 \\
\hline & & 1512 & 3192 \\
\hline & & 3528 & $3258^{\circ}$ \\
\hline & \multirow{5}{*}{250} & 204 & 300 \\
\hline & & 228 & 324 \\
\hline & & 252 & 372 \\
\hline & & 300 & 372 \\
\hline & & 324 & 444 \\
\hline
\end{tabular}

\subsubsection{Verificação gráfica da adequabilidade do modelo.}

Para verificar a adequabilidade do modelo (7.26) utilizamos um procedimento gráfico. Considerando as expressões (7.18), (7.19) e (7.21), isto é, fazemos o grafico dos erros "padronizados". versus os valores ajustados, estes valores encontram-se na tabela 8.12 , do apêndice c. Os erros "padronizados" se calculam com a seguinte fórmula $E R R O=$ $\widehat{\epsilon}_{i}^{*} /\left(s_{R} \sqrt{1-c_{i}}\right)$, onde $\widehat{\epsilon}_{i}^{*}$ e $s_{R}$ são dados pelas expressões $(7.19)$ e $(7.21)$ e $c_{i}=X^{\prime}\left(X^{\prime} X\right)^{-1} X$, para $i=1, \ldots, 20$. 


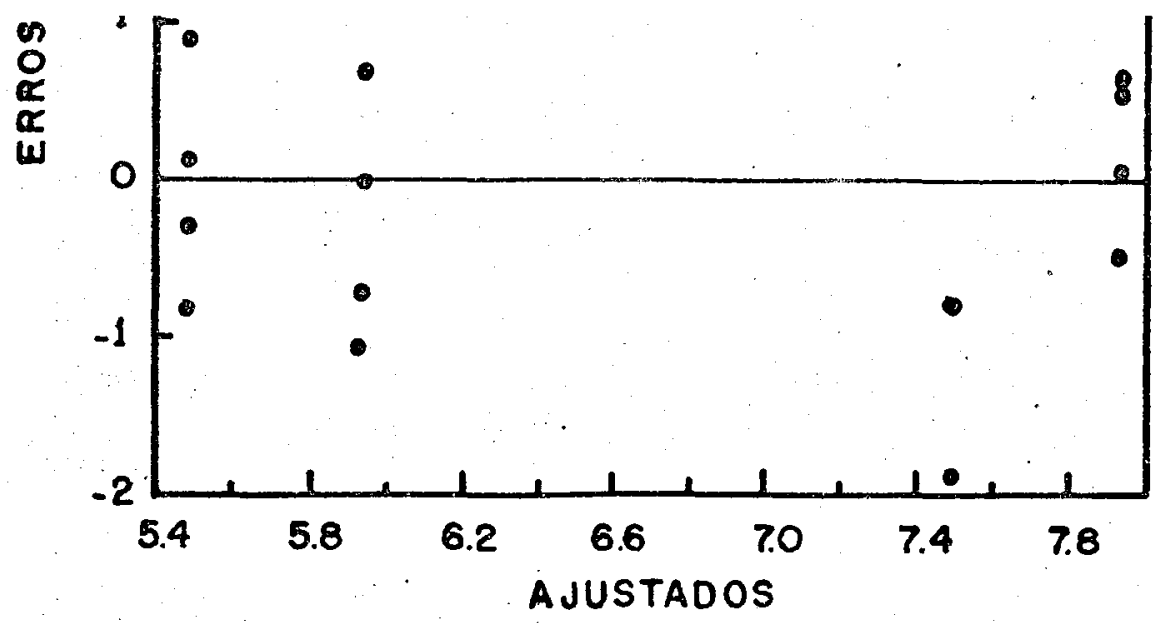

Figura 8.17 Gráfico dos erros "padronizados" versus valores ajustados.

No gráfico (8.17) podemos concluir que o modelo (7.26) é adequado para os dados da tabela 8.10. Cabe notar a presença de uma observação um pouco afastada, a qual merreceria uma análise mais minuiciosa, mas isto foge dos objetivos de nosso trabalho. Outras formas de analisar os erros do modelo (7.26) podem ser encontradas, por exemplo no livro de Price e Chatterjee, 1977.

\subsubsection{Análise clássica do problema.}

Utilizando a metodologia obtida no capítulo 7 realizamos uma análise clássica, dos dados da tabela 8.10. Nesta tabela temos os materiais I e II e duas temperaturas 200 e 250 graus centigrados, com 5 réplicas. Os EMQ para $\eta, \tau_{1}$ e $u_{1}$ são dados por $\widehat{\eta}^{* *}=6.7198(0.055 \mathrm{~S}), \widehat{\tau}_{1}^{* *}=1.0019(0.0558)$ e $\widehat{u}_{1}^{* *}=-0.2235(0.0558)$, isto empregando a expressão (7.33). Os valores entre parenteses são os respectivos desvios padrões estimados dos parâmetros considerando a expressão (7.34). Os intervalos de confiança $90 \%$ para $\eta$, $\tau_{1}$ e $u_{1}$ usando os EMQ e os desvios padrões estimados são $(6.6277 ; 6.8120),(0.9097 ; 1.0940)$ e $(-0.3156 ;-0.1314)$, respectivamente.

Os estimadores de máxima verossimilhança para $\eta, \tau_{1}, u_{1}$ e $\alpha$ obtidos numéri- 
camente são dados por $\hat{\eta}=6.7197(0.0527), \widehat{\tau}_{1}=1.0018(0.0527), \widehat{u}_{1}=-0.2169(0.0527)$ e $\hat{\alpha}=0.4652(0.1041)$. Os valores entre parenteses são os respectivos desvios padrões estimados dos parâmetros considerando a matriz de informação observada (7.42). Considerando a aproximação normal assintótica dos estimadores de máxima verossimilhança $\widehat{\eta}, \widehat{\tau}_{1}, \widehat{u}_{1}$ e $\widehat{\alpha}$ dados em (7.42) podemos construir intervalos de confiança $90 \%$ para $\eta, \tau_{1}, u_{1}$ e $\alpha$. Assim utilizando a matriz de informação observada, os intervalos de confiança $90 \%$ para $\eta, \tau_{1}, u_{1}$ e $\alpha$ são $(6.6327 ; 6.8067),(0.9148 ; 1.0887),(-0.3039 ;-0.1300)$ e $(0.2934 ; 0.6369)$, respectivamente.

\subsubsection{Análise bayesiana do problema.}

Considerando os dados da tabela 8.10, e utilizando a expressão (7.59), as modas para us parâmetros $\eta, \tau_{1}$ e $u_{1}$ são dadas por $\widehat{\eta}^{*}=6.7197, \widehat{\tau}_{1}^{*}=1.0025$ e $\widehat{u}_{1}^{*}=-0.2298$, as quais são obtidas por métodos numéricos.

Utilizando a aproximação normal os intervalos de credibilidade (calculados por métodos numéricos) para os parâmetros $\eta, \tau_{1}$ e $u_{1}$ são dadas por $(6.6328 ; 6.8067)$, $(0.9156 ; 1.0895)$ e $(-0.3167 ;-0.1428)$, respectivamente. 


\subsection{Conclusões}

Durante o desenvolvimento do trabalho foram obtidas várias conclusões pertinentes e, para sumarizar nos limitaremos às seguintes conclusões, específicas:

Utilizando o método de LAPLACE para aproximação de integrais em ambos modelos, obtivemos fórmulas muito simples e de fácil interpretação para as densidades a posteriori marginais e densidades preditivas de interesse.

Nos exemplos considerados (exemplo 1 e 2), utilizando os métodos Bayesianos, notamos uma boa precisão nas inferências dos resultados obtidos, o qual foi verificado numericamente (ver tabelas 8.4 e 8.8). Também é interessante observar que os resultados Bayesianos são equivalentes aos resultados clássicos, considerando as priori nãoinformativas usadas neste trabalho. Portanto, os métodos Bayesianos obtidos podem ser uma alternativa aos métodos clássicos assintóticos usuais considerados, para análise destes modelos. Ademais, na prática, considerando densidades a priori informativas e utilizando o método de LAPLACE obtivemos resultados mais precisos para a distribuição dos parâmetros. Isto implica que quando incorporamos informação a priori, pelos métodos bayesianos podemos ter inferências mais precisas.

Os procedimentos Bayesianos também permitem realizar inferências sobre o tempo médio de vida da fadiga. Além disso, utilizando a densidade preditiva de uma observação futura podemos formular testes de controle de qualidade. E ainda pelos métodos bayesianos é possivel realizar planejamentos de experimentos.

Embora os exemplos apresentados neste trabalho sejam casos particulares, eles são importantes porque os procedimentos bayesianos obtidos descrevem adequadamente situaçóes reais e podem ser aplicados, sempre que o valor de $\alpha$ seja pequeno $(0<\alpha<$ 1). É importante salientar que o uso de modelos paramétricos específicos só é possivel em engenharia quando os modelos descrevem adequadamente situações reais. Torna-se evidente a necessidade de um ambiente verdadeiramente inter-diciplinar, que possibilite a aplicação do conhecimento científico obtido, em projetos específicos, tanto na área de materiais, como em qualquer outra área de aplicação.

Finalmente, concluímos que, o uso de métodos Bayesianos para os modelos considerados podem ser de grande interesse prático na área de engenharia de materiais, devido a sua simplicidade e precisão dos resultados. 


\subsection{Direções para futuros trabalhos sobre a distri- buição de Birnbaum-Saunders.}

Realizar uma análise Bayesiana utilizando técnicas de regressão para o modelo Log-Linear da distribuição de Birnbaum-Saunders considerando duas variáveis independentes, pois nos experimentos das industrias de materiais que trabalham com fadigas podemos ter uma ou mais variáveis relacionadas entre si associadas às unidades experimentais. Uma das ferramentas mais utilizadas para a análise do relacionamento entre variáveis, é a análise de regressão, pois ela fornece um método simples para estabelecer um relacionamento funcional entre variáveis (esta análise está sendo finalizada, e será matéria de um relatório técnico).

Efetuar simulações para diversos tamanhos de amostras (estas simulações já estão sendo realizadas).

Estudar com profundidade o comportamento do parâmetro de forma $\alpha$, considerando diferentes valores para este parâmetro $(0<\alpha<1)$, com diversas amostras.

Desenvolvimento de um SOFTWARE para ser utilizado por pesquisadores na áréa de fadiga de materiais (isto está sendo realizado com a colaboração do prof. Dr. José Carlos Maldonado (ICMSC - USP)). 


\section{APENDICES}

\section{Apêndice $\mathbf{A}$}

\section{Método de aproximação de LAPLACE para integrais.}

A utilização de métodos Bayesianos usualmente exige cálculos (cálculos de integrais) envolvendo as densidades a posteriori e as densidades preditivas de interêsse. Estess cálculos em geral requerem métodos de aproximações numéricas, pois as soluções anàlíticas dessas integrais, em geral não são possíveis.

Um método muito utilizado em inferência Bayesiana aproximada é o método de LAPLACE para aproximação de integrais (ver por exemplo, Tierney e Kadane, 1986). O método de LAPLACE é um simples e eficaz método de expansão assintótica de integrais. Ou seja esta aproximação está baseada na distribuição normal (depois de desenvolver em serie de TAYLOR). Embora o ponto de vista seja Bayesiano os resultados são formais e tem aplicações para inferências não Bayesianas.

Supondo que $h(\varpi)$ seja uma função unimodal, pára aplicar o método, necessitamos maximizar a função $-h(\varpi)$, isto é encontrar $\widehat{\varpi}$ que maximiza $-h(\varpi)$.

Supor que temos interesse em resolver a integral em $\varpi$, dada por,

$$
I=\int f(\varpi) \exp [-n h(\varpi)] d \varpi .
$$


No caso uniparamétrico $(\varpi \epsilon R)$, se $\widehat{\varpi}$ é o valor que maximiza $-h(\varpi)$ em (A.1) e $\sigma=h^{\prime \prime}(\widehat{\varpi})^{-1 / 2}$, então o método de aproximação de LAPLACE é dado por,

$$
\widehat{I} \simeq(2 \pi / n)^{1 / 2} \sigma f(\widehat{\varpi}) \exp [-n h(\widehat{\varpi})] .
$$

No caso multiparamétrico, com $\varpi \epsilon R^{m}$, a aproximação de LAPLACE para (A.1) é dada por

$$
\widehat{I} \simeq(2 \pi)^{m / 2}\left[\operatorname{det}\left(n D^{2} h(\underline{\widehat{\widehat{\omega}}})\right)\right]^{-1 / 2} f(\underline{\widehat{\underline{\omega}}}) \exp [-n h(\underline{\widehat{\widehat{\omega}}})]
$$

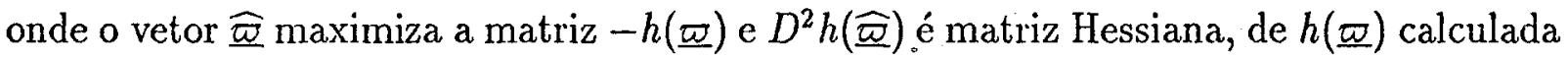
em $\widehat{\underline{\underline{ }} \text {. }}$

Um tratamento minucioso da teoría de aproximações de integrais de LAPLACE acima considerado, pode ser encontrado em Kass, Tierney e Ǩadane (1990).

Um caso especial de aproximação de LAPLACE para integrais da forma,

$$
\int e^{-n h(\varpi)} d \varpi
$$

pode ser encontrado em Tierney e Kadane 1986 e 1989. 


\section{Apêndice $B$}

Tabela 8.11 Valores de $i, t_{i}, 1 / i, F(\widehat{\alpha}, \widehat{\beta})$ e NSCORES, necessários para construir as figuras 6.1 e 6.2 .

\begin{tabular}{rrcccc}
\hline $\mathrm{i}$ & $t_{\mathrm{i}}$ & $\frac{\mathbf{1}}{i}$ & $F(\hat{\alpha}, \hat{\beta})$ & $\frac{(i-3 / 8)}{(101+1 / 4)}$ & NSCORES \\
\hline 1 & 70 & 0.0099 & 0.0001 & 0.0061 & -2.4972 \\
2 & 90 & 0.0198 & 0.0198 & 0.0160 & -2.1456 \\
3 & 96 & 0.0297 & 0.0307 & 0.0259 & -1.9475 \\
4 & 97 & 0.0396 & 0.0351 & 0.0358 & -1.8044 \\
5 & 99 & 0.0495 & 0.0455 & 0.0456 & -1.6905 \\
6 & 100 & 0.0594 & 0.0516 & 0.0555 & -1.5940 \\
7 & 103 & 0.0693 & 0.0735 & 0.0654 & -1.5117 \\
8 & 104 & 0.0792 & 0.0823 & 0.0753 & -1.4037 \\
9 & 104 & 0.0891 & 0.0823 & 0.0851 & -1.4037 \\
10 & 105 & 0.0990 & 0.0901 & 0.0950 & -1.3099 \\
11 & 107 & 0.1089 & 0.1093 & 0.1049 & -1.2533 \\
12 & 108 & 0.1188 & 0.1210 & 0.1148 & -1.1507 \\
13 & 108 & 0.1287 & 0.1210 & 0.1246 & -1.1507 \\
14 & 108 & 0.1386 & 0.1210 & 0.1345 & -1.1507 \\
15 & 109 & 0.1485 & 0.1314 & 0.1444 & -1.0374 \\
16 & 109 & 0.1584 & 0.1314 & 0.1543 & -1.0374 \\
17 & 112 & 0.1683 & 0.1685 & 0.1641 & -0.9557 \\
18 & 112 & 0.1782 & 0.1685 & 0.1740 & -0.9557 \\
19 & 113 & 0.1881 & 0.1841 & 0.1839 & -0.8984 \\
20 & 114 & 0.1980 & 0.1977 & 0.1938 & -0.8263 \\
21 & 114 & 0.2079 & 0.1977 & 0.2037 & -0.8263 \\
22 & 114 & 0.2178 & 0.1977 & 0.2136 & -0.8263 \\
23 & 116 & 0.2277 & 0.2266 & 0.2234 & -0.7584 \\
24 & 119 & 0.2376 & 0.2743 & 0.2333 & -0.7257 \\
25 & 120 & 0.2475 & 0.2912 & 0.2432 & -0.6626 \\
26 & 120 & 0.2574 & 0.2912 & 0.2530 & -0.6626 \\
27 & 120 & 0.2673 & 0.2912 & 0.2629 & -0.6626 \\
28 & 121 & 0.2772 & 0.3085 & 0.2728 & -0.5874 \\
29 & 121 & 0.2871 & 0.3085 & 0.2827 & -0.5874 \\
30 & 123 & 0.2970 & 0.3409 & 0.2925 & -0.5438 \\
& & & & &
\end{tabular}




\begin{tabular}{rrrrrr}
\hline $\mathrm{i}$ & $t_{i}$ & $\frac{1}{\mathrm{i}}$ & $\boldsymbol{F}(\widehat{\alpha}, \widehat{\beta})$ & $\frac{(i-3 / 8)}{(101+1 / 4)}$ & NSCORES \\
\hline 31 & 124 & 0.3069 & 0.3594 & 0.3024 & -0.4597 \\
32 & 124 & 0.3168 & 0.3594 & 0.3123 & -0.4597 \\
33 & 124 & 0.3267 & 0.3594 & 0.3222 & -0.4597 \\
34 & 124 & 0.3366 & 0.3594 & 0.3320 & -0.4597 \\
35 & 124 & 0.3465 & 0.3594 & 0.3419 & -0.4597 \\
36 & 128 & 0.3564 & 0.4325 & 0.3518 & -0.3655 \\
37 & 128 & 0.3663 & 0.4325 & 0.3617 & -0.3655 \\
38 & 129 & 0.3762 & 0.4483 & 0.3716 & -0.3132 \\
39 & 129 & 0.3861 & 0.4483 & 0.3814 & -0.3132 \\
40 & 130 & 0.3960 & 0.4681 & 0.3913 & -0.2490 \\
41 & 130 & 0.4059 & 0.4681 & 0.4012 & -0.2490 \\
42 & 130 & 0.4158 & 0.4681 & 0.4111 & -0.2490 \\
43 & 131 & 0.4257 & 0.4840 & 0.4209 & -0.1484 \\
44 & 131 & 0.4356 & 0.4840 & 0.4308 & -0.1484 \\
45 & 131 & 0.4455 & 0.4840 & 0.4407 & -0.1484 \\
46 & 131 & 0.4554 & 0.4840 & 0.4506 & -0.1484 \\
47 & 131 & 0.4653 & 0.4840 & 0.4604 & -0.1484 \\
48 & 132 & 0.4752 & 0.5040 & 0.4703 & -0.0493 \\
49 & 132 & 0.4851 & 0.5040 & 0.4802 & -0.0493 \\
50 & 132 & 0.4950 & 0.5040 & 0.4901 & -0.0493 \\
51 & 133 & 0.5050 & 0.5199 & 0.5000 & 0.0000 \\
52 & 134 & 0.5149 & 0.5398 & 0.5098 & 0.0740 \\
53 & 134 & 0.5248 & 0.5398 & 0.5197 & 0.0740 \\
54 & 134 & 0.5347 & 0.5398 & 0.5296 & 0.0740 \\
55 & 134 & 0.5446 & 0.5398 & 0.5395 & 0.0740 \\
56 & 134 & 0.5545 & 0.5398 & 0.5493 & 0.0740 \\
57 & 136 & 0.5644 & 0.5714 & 0.5592 & 0.1609 \\
58 & 136 & 0.5743 & 0.5714 & 0.5691 & 0.1609 \\
59 & 137 & 0.5842 & 0.5910 & 0.5790 & 0.1984 \\
60 & 138 & 0.5941 & 0.6064 & 0.5888 & 0.2490 \\
61 & 138 & 0.6040 & 0.6064 & 0.5987 & 0.2490 \\
62 & 138 & 0.6139 & 0.6064 & 0.6086 & 0.2490 \\
63 & 139 & 0.6238 & 0.6217 & 0.6185 & 0.3132 \\
64 & 139 & 0.6337 & 0.6217 & 0.6283 & 0.3132 \\
65 & 141 & 0.6436 & 0.6554 & 0.6382 & 0.3655 \\
66 & 141 & 0.6535 & 0.6554 & 0.6481 & 0.3655 \\
& & & & &
\end{tabular}




\begin{tabular}{rrrrrr}
\hline $\mathrm{i}$ & $t_{i}$ & $\frac{1}{i}$ & $F(\hat{\alpha}, \widehat{\beta})$ & $\frac{(i-3 / 8)}{(101+1 / 4)}$ & NSCORES \\
\hline 67 & 142 & 0.6634 & 0.6700 & 0.6580 & 0.4734 \\
68 & 142 & 0.6733 & 0.6700 & 0.6679 & 0.4734 \\
69 & 142 & 0.6832 & 0.6700 & 0.6777 & 0.4734 \\
70 & 142 & 0.6931 & 0.6700 & 0.6876 & 0.4734 \\
71 & 142 & 0.7030 & 0.6700 & 0.6975 & 0.4734 \\
72 & 142 & 0.7129 & 0.6700 & 0.7074 & 0.4734 \\
73 & 144 & 0.7228 & 0.6985 & 0.7172 & 0.5874 \\
74 & 144 & 0.7327 & 0.6985 & 0.7271 & 0.5874 \\
75 & 145 & 0.7426 & 0.7123 & 0.7370 & 0.6321 \\
76 & 146 & 0.7525 & 0.7257 & 0.7469 & 0.6626 \\
77 & 148 & 0.7624 & 0.7517 & 0.7567 & 0.7097 \\
78 & 148 & 0.7723 & 0.7517 & 0.7666 & 0.7097 \\
79 & 149 & 0.7822 & 0.7642 & 0.7765 & 0.7584 \\
80 & 151 & 0.7921 & 0.7881 & 0.7864 & 0.8090 \\
81 & 151 & 0.8020 & 0.7881 & 0.7962 & 0.8090 \\
82 & 152 & 0.8119 & 0.7995 & 0.8061 & 0.8618 \\
83 & 155 & 0.8218 & 0.8289 & 0.8160 & 0.8984 \\
84 & 156 & 0.8317 & 0.8389 & 0.8259 & 0.9362 \\
85 & 157 & 0.8416 & 0.8485 & 0.8358 & 1.0374 \\
86 & 157 & 0.8515 & 0.8485 & 0.8456 & 1.0374 \\
87 & 157 & 0.8614 & 0.8485 & 0.8555 & 1.0374 \\
88 & 157 & 0.8713 & 0.8485 & 0.8654 & 1.0374 \\
89 & 158 & 0.8812 & 0.8557 & 0.8753 & 1.1507 \\
90 & 159 & 0.8911 & 0.8643 & 0.8851 & 1.2004 \\
91 & 162 & 0.9010 & 0.8869 & 0.8950 & 1.2533 \\
92 & 163 & 0.9109 & 0.8944 & 0.9049 & 1.3399 \\
93 & 163 & 0.9208 & 0.8944 & 0.9148 & 1.3399 \\
94 & 164 & 0.9307 & 0.8987 & 0.9246 & 1.4379 \\
95 & 166 & 0.9406 & 0.9131 & 0.9345 & 1.5520 \\
96 & 166 & 0.9505 & 0.9131 & 0.9444 & 1.5520 \\
97 & 168 & 0.9604 & 0.9236 & 0.9543 & 1.6905 \\
98 & 170 & 0.9703 & 0.9332 & 0.9641 & 1.8044 \\
99 & 174 & 0.9802 & 0.9484 & 0.9740 & 1.9475 \\
100 & 196 & 0.9901 & 0.9904 & 0.9839 & 2.1456 \\
101 & 212 & 1.0000 & 0.9976 & 0.9938 & 2.4972 \\
\hline & & & & &
\end{tabular}




\section{Apêndice $\mathrm{C}$}

Tabela 8.12 Valores de $i$, valores observados $(\underline{Y})$, valores ajustados $(\underline{\hat{Y}})$, erros $\left(\underline{\widehat{\epsilon}}^{*}\right) e$ erros "padronizados" (ERRO).

\begin{tabular}{ccccc}
\hline $\mathrm{i}$ & $\underline{Y}$ & $\underline{\hat{Y}}$ & $\widehat{\underline{\epsilon}}^{*}$ & ERRO \\
\hline 1 & 7.0699 & 7.4982 & -0.4283 & -1.86 \\
2 & 7.3212 & 7.4982 & -0.1770 & -0.77 \\
3 & 7.3212 & 7.4982 & -0.1770 & -0.77 \\
4 & 7.3212 & 7.4982 & -0.1770 & -0.77 \\
5 & 8.1685 & 7.4982 & 0.6703 & 2.91 \\
6 & 7.8320 & 7.9452 & -0.1131 & -0.49 \\
7 & 7.9572 & 7.9452 & 0.0120 & 0.05 \\
8 & 8.0684 & 7.9452 & 0.1232 & 0.54 \\
9 & 8.0684 & 7.9452 & 0.1232 & 0.54 \\
10 & 8.0889 & 7.9452 & 0.1437 & 0.62 \\
11 & 5.3181 & 5.4945 & -0.1764 & -0.77 \\
12 & 5.4293 & 5.4945 & -0.0651 & -0.28 \\
13 & 5.5294 & 5.4945 & 0.0350 & 0.15 \\
14 & 5.7038 & 5.4945 & 0.2093 & 0.91 \\
15 & 5.7807 & 5.4945 & 0.2863 & 1.24 \\
16 & 5.7038 & 5.9414 & -0.2377 & -1.03 \\
17 & 5.7807 & 5.9414 & -0.1607 & -0.70 \\
18 & 5.9189 & 5.9414 & -0.0225 & -0.10 \\
19 & 5.9189 & 5.9414 & -0.0225 & -0.10 \\
20 & 6.0958 & 5.9414 & 0.1544 & 0.67 \\
\hline
\end{tabular}




\section{Bibliografía}

ACHCAR, J.A. (1990) - Use of predictive densities in quality control with accelerated life tests assuming a power rule model and an exponential distribution. São Carlos, ICMSC-USP, 1990, S.P. (Notas do ICMSC-USP, 76).

ACHCAR, J.A. (1991) - Inference for the Birnbaum-Saunders fatigue life model using Bayesian methods. São Carlos, ICMSC-USP, 1991, S.P. 16 p. (Notas do ICMSC-USP, 86).

ACHCAR, J.A. ; MARTINEZ ESPINOSA, M. (1992) - Use of Bayesian methods in : accelerated life tests considering a Log-Linear model for the Birnbaum-Saunders distribution. São Carlos, ICMSC-USP, 1992, S.P. (Notas do ICMSC-USP, 105).

BIRNBAUM, S.W. ; SAUNDERS, S.C. (1958) - A statistical model for life-length of materials. Journal of the American Statistical Association, v. 53, p. 151-60, 1958.

BIRNBAUM, S.W. ; SAUNDERS, S.C. (1968) - A probabilistic interpretation of Miner's rule. Siam J. Appl. Math. v.16, p. 637-52, 1968.

BIRNBAUM, S.W. ; SAUNDERS, S.C. (1969-a) - A New Family of life distribution. Journal of Applied Probability, v. 6, p. 319-27, 1969.

BIRNBAUM, S.W. ; SAUNDERS; S.C. (1969-b) - Estimation for a Family of life distribution com Application to Fatigue. Journal of Applied Probability, v. 6, p. 328-47, 1969.

BOX, G.E.P. ; TIAO, G.C. (1973) - Bayesian Inference in Statistical Analysis. Reading. Addisson-Wesley, 1973. 
BROWN, M.W. ; MILLER, K.J. (1978) - Baixial Fatigue data. Sheffield, University of Sheffield - Dept. of mechanical engineering. (Report CEMR 1/78), 1978.

CARVALHO, J.F.; DACHS, J.N. (1982) - Regressão. São Carlos, UFSCAR, 1982 S.P. (Notas manuscritas).

COX, D.R.; REID, N. (1987) - Ortogonal Parameters and Aproximated condicional inference with discussion, Journal Royal Statistical Society B. v. 49, N. 1, p. 1-39, 1987.

DACHS, J. N. W. (1988) - Estatística computacional, uma introdução em Turbo Pascal. Rio de Janeiro, Ltd, 1988.

ENGELHARDT, M ; BAIN, L.J. ; WRIGHT, F.T. (1981) - Inferences on the Parameters of the Brinbaum-Saunders Fatigue Life distribution based on maximun likelihood estimation. Technometrics, v. 23, p. 251-6, 1981.

FRIES, A. ; BHATTACHARYYA, G.K. (1983) - Analysis of Two-Factor Experimenter under an Inverse Gaussian model. Journal of the American Statistical Association, v. 78 , p. 820-6, 1983.

GUTTMAN, I. (1982) - Linear Models: An Introduction." New York, John Wiley, 1982.

KALBFLEISCH, J.G. (1985) - Probability and Statistical Inference. 2 ed. New York, Springer-Verlag, 1985. v.2: Statistical Inference.

KASS, R.E.; TIERNEY, L.; KADANE, J.B. (1990) - The validity of posterior expansions based on Laplace's method. In: Geisser, S et al. - Bayesian and Likelihood Methods in Statistics and Econometrics: Essays in honor of George A. Barnard. Amsterdam, North-Holland, 1990. p. 473-88.

LAWLESS, J.F. (1982) - Statistical Models and Methods for life time data. New York, John Wiley, 1982.

LEITHOLD, L. (1972) - The calculus whith analytic geometry. New York, Harpers \& Row, 1972.

LLORCA, F.J.S; LOZANO, A. M. (1980) - Programación con el lenguaje Pascal. Madrid, Paraninfo, 1980. 
MANN,N.R;SCHAFFER, R.E; SINGPURWALLA, N.D. (1974) - Methods for Siatis tical Analysis of Reability and Lifetime data. New York, John Wiley, 1974.

MILLER, R.G. (1981) - Survival Analysis. New York, John Wiley, 1981.

MOOD, A.M.; GRAYBILL, F.A.; BOES, D.C. (1974) - Introduction to the Theory of Statistics. 3 ed. New York, McGraw-Hill, 1974.

NELSON, W. (1990) - Accelerated Testing: Statistical models, test plans, and data analyses. New York, John Wiley, 1990.

PRESS, S. J. (1989) - Bayesian Statistics: Principles, Models and Applications. New York, John Wiley, 1989.

PRICE, B. ; CHATTERJEE, S. (1977) - Regression Analysis by Example. New York, John Wiley, 1977.

RIECK, J.R. ; NEDELMAN, J.R. (1991) - A Log-Linear Model for the BirnbaumSaunders distribution. Technometrics, v. 33, p. 51-60, 1991.

ROSS, S. (1976) - A first course in Probability. New York, Macmillan, 1976.

SCHEFFÉ, H. (1959) - The analysis of Variance. New York, John Wiley, 1959.

SEARLE, S.R. (1971) - Linear models. New York, John Wiley, 1971.

SOUZA; S.A. (1982) - Ensaios mecânicos de materiais metálicos: Fundamentos teóricos e práticos. São Paulo, Edgard Blucher, 1982.

TIBSHIRANI, R. (1989) - Noninformative priors for one parameter of many. Biometrika, v. 76, p. $604-8,1989$.

TIERNEY, L. ; KADANE, J.B. (1986) - Accurate Approximations for posterior moments and marginal densities. Journal of the American Statistical Association, v, 81, p. $82-6,1986$.

ZELLNER,A. (1971) - An Introduction to Bayesicn Inference in Econometrics. New York, John Wiley, 1971. 
"Se alguém necessita de sabedoria peça a Deus que a todos da liberalmente " 